\title{
Topological Insulator Materials
}

\author{
Yoichi ANDo* \\ Institute of Scientific and Industrial Research, Osaka University, Ibaraki, Osaka 567-0047, Japan
}

\begin{abstract}
Topological insulators represent a new quantum state of matter which is characterized by peculiar edge or surface states that show up due to a topological character of the bulk wave functions. This review presents a pedagogical account on topological insulator materials with an emphasis on basic theory and materials properties. After presenting a historical perspective and basic theories of topological insulators, it discusses all the topological insulator materials discovered as of May 2013, with some illustrative descriptions of the developments in materials discoveries in which the author was involved. A summary is given for possible ways to confirm the topological nature in a candidate material. Various synthesis techniques as well as the defect chemistry that are important for realizing bulk-insulating samples are discussed. Characteristic properties of topological insulators are discussed with an emphasis on transport properties. In particular, the Dirac fermion physics and the resulting peculiar quantum oscillation patterns are discussed in detail. It is emphasized that proper analyses of quantum oscillations make it possible to unambiguously identify surface Dirac fermions through transport measurements. The prospects of topological insulator materials for elucidating novel quantum phenomena that await discovery conclude the review.
\end{abstract}

KEYWORDS: topological insulator, Dirac fermions, surface state, quantum oscillations

\section{Introduction}

The progress in condensed matter physics is often driven by discoveries of novel materials. In this regard, materials presenting unique quantum-mechanical properties are of particular importance. Topological insulators (TIs) are a class of such materials and they are currently creating a surge of research activities. ${ }^{1-3)}$ Because TIs concern a qualitatively new aspect of quantum mechanics, i.e. the topology of the Hilbert space, they opened a new window for understanding the elaborate workings of nature.

TIs are called "topological" because the wave functions describing their electronic states span a Hilbert space that has a nontrivial topology. Remember, quantum-mechanical wave functions are described by linear combinations of orthonormal vectors forming a basis set, and the abstract space spanned by this orthonormal basis is called Hilbert space. In crystalline solids, where the wave vector $\mathbf{k}$ becomes a good quantum number, the wave function can be viewed as a mapping from the $\mathbf{k}$-space to a manifold in the Hilbert space (or in its projection), and hence the topology becomes relevant to electronic states in solids. Depending on the way the Hilbertspace topology becomes nontrivial, there can be various different kinds of TIs. ${ }^{4)}$ An important consequence of a nontrivial topology associated with the wave functions of an insulator is that a gapless interface state necessarily shows up when the insulator is physically terminated and faces an ordinary insulator (including the vacuum). This is because the nontrivial topology is a discrete characteristic of gapped energy states, and as long as the energy gap remains open, the topology cannot change; hence, in order for the topology to change across the interface into a trivial one, the gap must close at the interface. Therefore, three-dimensional (3D) TIs are always associated with gapless surface states, and so are two-dimensional (2D) TIs with gapless edge states. This principle for the necessary occurrence of gapless interface states is called bulkboundary correspondence in topological phases.

A large part of the unique quantum-mechanical proper- (a)

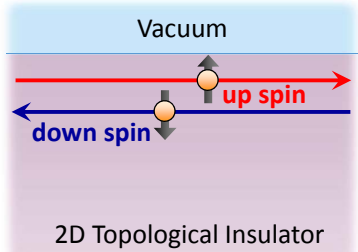

(b)

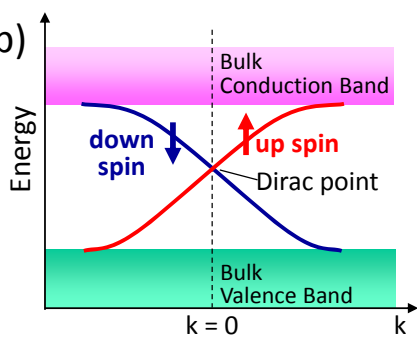

(c)
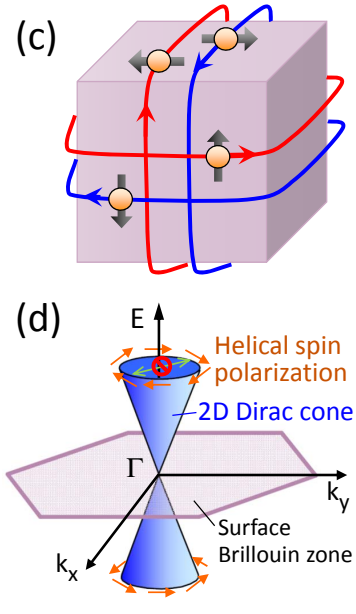

Fig. 1. (Color online) Edge and surface states of topological insulators with Dirac dispersions. (a) Schematic real-space picture of the 1D helical edge state of a 2D TI. (b) Energy dispersion of the spin non-degenerate edge state of a 2D TI forming a 1D Dirac cone. (c) Schematic real-space picture of the 2D helical surface state of a 3D TI. (d) Energy dispersion of the spin non-degenerate surface state of a 3D TI forming a 2D Dirac cone; due to the helical spin polarization, back scattering from $\mathbf{k}$ to $-\mathbf{k}$ is prohibited. 
ties of TIs come from the peculiar characteristics of the edge/surface states. Currently, the TI research is focused mostly on time-reversal (TR) invariant systems, where the nontrivial topology is protected by time-reversal symmetry (TRS). ${ }^{1-3)}$ In those systems, the edge/surface states present Dirac dispersions (Fig. 1), and hence the physics of relativistic Dirac fermions becomes relevant. Furthermore, spin degeneracy is lifted in the Dirac fermions residing in the edge/surface states of TR-invariant TIs and their spin is locked to the momentum (Fig. 1). Such a spin state is said to have "helical spin polarization" and it provides an opportunity to realize Majorana fermions ${ }^{5)}$ in the presence of proximity-induced superconductivity, ${ }^{6)}$ not to mention its obvious implications for spintronics applications. An important objective of the experimental studies of TIs has been to elucidate the existence and the nature of such helically spin-polarized Dirac fermions in the topological surface state.

This review is aimed at providing a pedagogical introduction to the field of topological insulators, putting emphasis on the basic theory and materials properties. It also elaborates on the basics of the characterizations of TI materials using transport measurements.

\section{Historical Perspective}

It is useful to understand the historical background in order to appreciate the importance of TIs in condensed matter physics. In this section, I will briefly discuss several relevant issues that preceded the discovery of TIs and also describe how the TI research developed in its early days.

\subsection{Integer quantum Hall effect}

In 1980, von Klitzing et al. discovered the quantum Hall effect in a high-mobility 2D semiconductor under high magnetic fields. ${ }^{7}$ The occurrence of this effect is usually limited to very low temperatures, where localization of electrons and Landau quantization of their energy spectrum lead to vanishing longitudinal conductivity $\sigma_{x x}$ together with quantization of the Hall conductivity $\sigma_{x y}$ to integer multiples of $e^{2} / h$ when the chemical potential is located in between Landau levels. Such a quantization of transport coefficients obviously pointed to a macroscopic quantum phenomenon, as was made clear by Laughlin's gauge argument. ${ }^{8)}$ It is prudent to mention that this quantization phenomenon was theoretically anticipated already in 1974. ${ }^{9}$ ) In 1982, it was recognized by Thouless, Kohmoto, Nightingale, and den Nijs (TKNN) ${ }^{10)}$ that this phenomenon not only is quantum mechanical but also is topological; namely, TKNN showed ${ }^{10)}$ that in the quantum Hall system the k-space is mapped to a topologically-nontrivial Hilbert space, whose topology can be specified by an integer topological invariant called TKNN invariant $v$, and that $\sigma_{x y}$ becomes equal to $v$ times $e^{2} / h$. The TKNN invariant is also called the first Chern number or the winding number, and it is equal to the Berry phase of the Bloch wave function calculated around the Brillouin zone (BZ) boundary divided by $2 \pi$ (actual calculations are shown in the next section).
In hindsight, the quantum Hall system can be considered to be the first topological insulator that became known to physicists, because when the quantization is taking place, the energy spectrum is gapped due to the Landau quantization and the chemical potential is located within the gap, which is a situation akin to an insulator. In this case, the nontrivial topology specified by the TKNN invariant is characteristic of a 2D system with broken TRS. Also, as was shown by Halperin, ${ }^{11)}$ the integer quantum Hall effect is always accompanied by chiral edge states, and those gapless states residing at the interface to the vacuum can be understood to be a result of the bulkboundary correspondence due to the topological 2D "bulk" state.

It is prudent to mention that the integer quantum Hall effect was a tip of an iceberg. The fractional quantum Hall (FQH) effect discovered in 1982 by Tsui, Stormer, and Gossard ${ }^{12}$ ) turned out to contain richer physics, because electron correlations play essential roles in the FQH effect and they lead to the appearance of fractionally-charged quasiparticles. ${ }^{13)}$ In terms of topology, however, FQH states do not have much relevance to topological insulators, because the former present groundstate degeneracy and their topological character is described by quite an abstract concept of topological order. ${ }^{14)}$

\subsection{Quantum spin Hall effect and $Z_{2}$ topology}

On a different front in condensed matter physics, generation and manipulation of spin currents have been attracting a lot of interest, since they will have a profound impact on future spintronics. ${ }^{15)}$ In this regard, the spin Hall effect, the appearance of transverse spin current in response to longitudinal electric field, has been discussed theoretically since $1970 \mathrm{~s},{ }^{16-20)}$ but its experimental confirmation by Kato et al. ${ }^{21)}$ in 2004 gave a big boost to the research of this phenomenon. It was soon recognized that the spin Hall effect in nonmagnetic systems is fundamentally related to the anomalous Hall effect in ferromagnets, ${ }^{22)}$ and similarly to the latter effect, there are both intrinsic and extrinsic origins of the spin Hall effect. The intrinsic mechanism of the spin Hall effect stems from the Berry curvature of the valence-band Bloch wave functions integrated over the Brillouin zone. ${ }^{19,20)}$ Since such an integral can become finite even in an insulator, Murakami, Nagaosa, and Zhang went on to propose the idea of spin Hall insulator ${ }^{23)}$ which is a gapped insulator with zero charge conductivity but has a finite spin Hall conductivity due to a finite Berry phase of the occupied states.

Later it was shown ${ }^{24)}$ that the proposed spin Hall insulators cannot really generate spin currents in the absence of any electrons at the Fermi level, but this idea triggered subsequent proposals of its quantized version, the quantum spin Hall (QSH) insulator, by Kane and Mele, ${ }^{25,26)}$ followed by an independent proposal by Bernevig and Zhang. ${ }^{27)}$ The QSH insulators are essentially two copies of the quantum Hall system, in which the chiral edge state is spin polarized and the two states form a time-reversed pair to recover the overall TRS. When current 
flows using the edge states of a QSH insulator, a quantized version of the spin Hall effect, the QSH effect, is predicted to be observed. Since the predicted phenomenon is based on the quantum Hall effect, it only exists in 2D. While it is not a priori clear how one can achieve such a state with quantized edge states in zero magnetic field, the ingenious proposal by Kane and Mele provided a concrete model to realize the QSH insulator. ${ }^{25)}$ Their model is essentially a graphene model with spin-orbit coupling (SOC).

In graphene, the band structure near the Fermi level consists of two linearly dispersing cones located at $K$ and $K^{\prime}$ points in the $\mathrm{BZ} ;{ }^{28)}$ since the low-energy physics on these cones is described by employing the Dirac equation with the rest mass set to zero, ${ }^{29}$ ) this dispersion is called Dirac cone and the electrons are said to behave as massless Dirac fermions. Kane and Mele showed ${ }^{25}$ ) that a finite SOC leads to an opening of a gap at the crossing point of the cone (called Dirac point) and, furthermore, that a time-reversed pair of spin-polarized one-dimensional (1D) states indeed show up at the edge in some parameter range; in this model, the desired spin polarization of the edge state is achieved due to the SOC which has an inherent tendency to align spins in relation to the momentum direction. This peculiar spin-non-degenerate state [Fig. 1(a,b)] is often said to have helical spin polarization or spin-momentum locking. Intriguingly, those electrons in the gapless edge state behave as 1D massless Dirac fermions within the gap opened in the 2D Dirac cone. In this case, the 2D "bulk" electrons can be viewed as massive Dirac fermions because of the finite energy gap at the Dirac point.

Most importantly, Kane and Mele recognized that the electronic states of their QSH insulator is characterized by a novel topology specified by a $Z_{2}$ index, ${ }^{26)}$ which expresses whether the number of times the 1D edge state crosses the Fermi level between 0 and $\pi / a$ is even or odd ( $a$ is the lattice constant). Remember, in mathematics the group of integer numbers is called $Z$ and its quotient group classifying even and odd numbers is called $Z_{2}$; hence, a $Z_{2}$ index generally gives a topological classification based on parity. (A detailed description of the $Z_{2}$ index for TR-invariant TIs is given in the next section.) The theoretical discovery of the $Z_{2}$ topology in insulators was a big step in our understanding of topological phases of matter, because it indicated that nontrivial topology can be embedded in the band structure of an ordinary insulator and that breaking of TRS by application of magnetic fields is not mandatory for realizing a topological phase.

Unfortunately, the SOC in graphene is very weak, and hence it is difficult to experimentally observe the QSH effect predicted in the Kane-Mele model. However, another theoretical breakthrough was soon made by Bernevig, Hughes, and Zhang (BHZ) ${ }^{30)}$ who constructed a $2 \mathrm{D}$ model to produce a $Z_{2}$ topological phase based on the band structure of HgTe; based on their model, BHZ predicted that a $\mathrm{CdTe} / \mathrm{HgTe} / \mathrm{CdTe}$ quantum well should give rise to the QSH effect. This prediction was verified in 2007 by König et al., ${ }^{31)}$ who observed

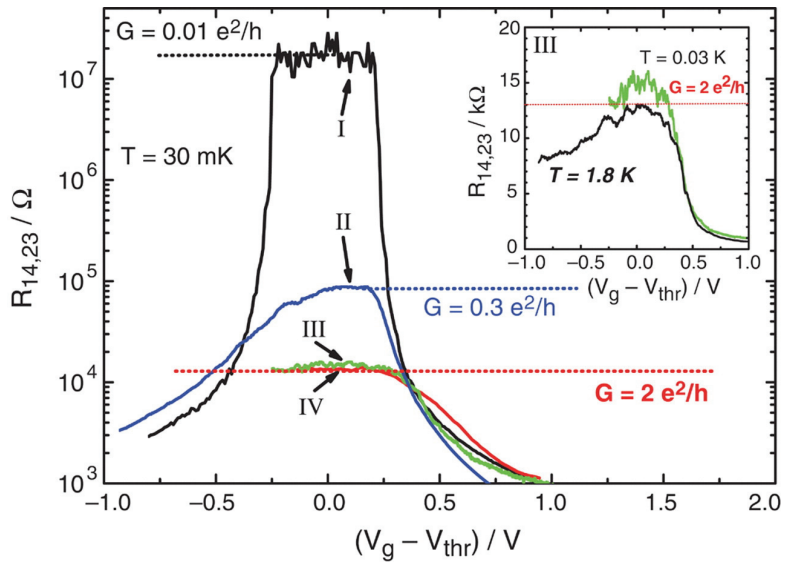

Fig. 2. (Color online) Longitudinal four-terminal resistance of various $\mathrm{CdTe} / \mathrm{HgTe} / \mathrm{CdTe}$ quantum-well structures as a function of the gate voltage measured in zero magnetic field at $30 \mathrm{mK}$. Devices with the size of $1 \times 1 \mu \mathrm{m}^{2}$ or less in the band-inverted regime (III and IV) show quantized conductance of $2 e^{2} / h$, giving evidence for the 2D TR-invariant TI phase. Taken from Ref. 31; copyright American Association for the Advancement of Science (2007).

$\sigma_{x x}$ to be quantized to $2 e^{2} / h$ in zero magnetic field when the chemical potential is tuned into the bulk band gap (Fig. 2), giving evidence for the gapless edge states in the insulting regime. This was the first experimental confirmation of the TR-invariant TI characterized by the $Z_{2}$ topology.

Without waiting for experimental verification of the $Z_{2}$ topology in $2 \mathrm{D}$, theorists noticed that this topological classification of insulators can be extended to 3D systems, where there are four $Z_{2}$ invariants to fully characterize the topology. ${ }^{32-34)}$ In fact, the term "topological insulator" was coined by Moore and Balents in their paper to propose the existence of TIs in 3D systems. ${ }^{32}$ ) For 3D TIs, Fu and Kane made a concrete prediction in 2006 that the $\mathrm{Bi}_{1-x} \mathrm{Sb}_{x}$ alloy in the insulating composition should be a TI, and they further proposed that the nontrivial topology can be verified by looking at the surface states using the angle-resolved photoemission spectroscopy (ARPES) and counting the number of times the surface states cross the Fermi energy between two TR-invariant momenta. ${ }^{35)}$ The proposed experiment was conducted by Hsieh et al. who reported in 2008 that $\mathrm{Bi}_{1-x} \mathrm{Sb}_{x}$ is indeed a $3 \mathrm{D}$ TI. ${ }^{36)}$ The experimental identification of $\mathrm{Bi}_{1-x} \mathrm{Sb}_{x}$ as a TR-invariant TI opened a lot of new experimental opportunities to address a topological phase of matter. For example, the first transport study of $\mathrm{Bi}_{1-x} \mathrm{Sb}_{x}$ to detect topological 2D transport channels was reported by Taskin and Ando, ${ }^{37}$ and the first scanning tunneling spectroscopy (STS) study that addressed the peculiar spin polarization was reported by Roushan et al. ${ }^{38)}$ both in 2009. Direct observation of the helical spin polarization of the surface states in $\mathrm{Bi}_{1-x} \mathrm{Sb}_{x}$ using spin-resolved ARPES was first partially done by Hsieh et $a l .{ }^{39)}$ and then fully accomplished by Nishide et al. (Fig. 3). ${ }^{40)}$ 


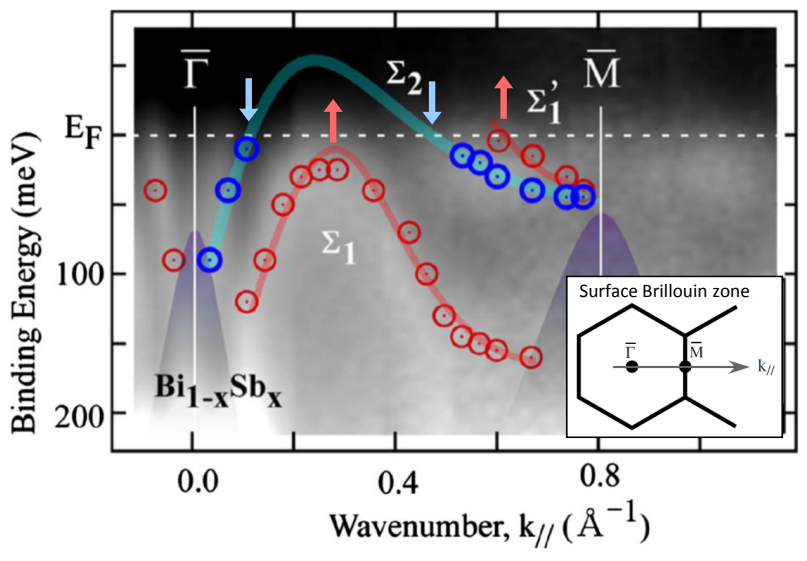

Fig. 3. (Color online) Spin-resolved surface band structure of $\mathrm{Bi}_{1-x} \mathrm{Sb}_{x}$ ( $x=0.12-0.13)$ on the (111) cleaved surface. Its surface BZ and the momentum direction of the data are shown in the inset. Dispersions shown by symbols are determined from the peak positions of the spin-resolved energy dispersion curves and they are plotted on top of the spin-integrated ARPES data shown in gray scale. There are three Fermi-level crossings of the surface states, which signifies the $Z_{2}$-nontrivial nature of this system. Taken from Ref. 40; copyright American Physical Society (2009).

\subsection{Topological field theory}

While the notion of topological insulators became popular only after the discovery of the $Z_{2}$ topology by Kane and Mele ${ }^{26)}$ there had been theoretical efforts to conceive topological states of matter beyond the scope of the quantum Hall system. In this respect, an important development was made in 2001 by Zhang and $\mathrm{Hu}$, who generalized the 2D quantum Hall state to a four-dimensional (4D) TR-invariant state possessing an integer topological invariant. ${ }^{41)}$ The effective field theory for this 4D topological system was constructed by Bernevig et al. ${ }^{42)}$ After the $Z_{2}$ topology was discovered for TR-invariant systems in $2 \mathrm{D}$ and $3 \mathrm{D},{ }^{26,32-34)}$ it was shown by Qi, Hughes, and Zhang ${ }^{43)}$ that the framework of topological field theory is useful for describing those systems as well, and they further demonstrated that the $Z_{2}$ TIs in $2 \mathrm{D}$ and $3 \mathrm{D}$ can actually be deduced from the 4D effective field theory by using the dimensional reduction. From a practical point of view, the topological field theory is suitable for describing the electromagnetic response of TIs and has been used for predicting novel topological magnetoelectric effects. ${ }^{43-46)}$

\subsection{Dirac materials}

It is interesting to note that the first 3D TI material $\mathrm{Bi}_{1-x} \mathrm{Sb}_{x}$, whose topological surface state consists of $2 \mathrm{D}$ massless Dirac fermions, has long been known to possess peculiar band structure to give rise to 3D massive Dirac fermions in the bulk. ${ }^{47}$ This situation is similar to the Kane-Mele model where 1D massless Dirac fermions emerge out of 2D massive Dirac fermions. Since Dirac fermions play important roles in TIs, it would be useful to mention the history of Dirac physics in condensed matter.
The semimetal Bi has been an important testing ground of quantum physics ever since the Shubnikov-de Haas ( $\mathrm{SdH})$ and de Haas-van Alphen (dHvA) oscillations were discovered in $\mathrm{Bi}$ in 1930. ${ }^{48)}$ This is essentially because the extremely low carrier density $\left(\sim 10^{-5}\right.$ per atom) and the very long mean free path (reaching $\sim 1 \mathrm{~mm}$ ) easily put the system in the "quantum limit" at relatively low magnetic fields. ${ }^{49)}$ In the mid-20th century, one of the long-standing puzzles in $\mathrm{Bi}$ was its large diamagnetism, which defies the common wisdom for magnetism in metals involving Pauli paramagnetism and Landau diamagnetism. ${ }^{50)}$ Intriguingly, in $\mathrm{Bi}_{1-x} \mathrm{Sb}_{x}$ at low $\mathrm{Sb}$ concentration, the carrier density becomes even lower than in $\mathrm{Bi}$, while the diamagnetic susceptibility increases, which is also opposite to the expectation from Landau diamagnetism. ${ }^{50)}$ To address the unusual electronic properties of $\mathrm{Bi}$, an effective two-band model was constructed by Cohen and Blount in 1960.51) In 1964, Wolff recognized that this two-band model can be transformed into the four-component massive Dirac Hamiltonian, and he presented an elegant analysis of the selection rules using the Dirac theory. ${ }^{47}$ ) This was the beginning of the notion of Dirac fermions in solid states, although some of the peculiar physics of massless Dirac fermions were recognized in as early as 1956 by McClure in the context of graphite. ${ }^{52)}$

Speaking of graphite, the mapping of the $\mathbf{k} \cdot \mathbf{p}$ Hamiltonian of its 2D sheet (i.e. graphene) ${ }^{53)}$ to the massless Dirac Hamiltonian was first used by Semenoff in $1984 .{ }^{54)}$ With the experimental realization of graphene, ${ }^{28)}$ this system has become a prototypical Dirac material. One of the distinguishing properties of massless Dirac fermions is the Berry phase of $\pi$; an important consequence of the $\pi$ Berry phase in the condensed matter setting is the absence of backscattering, which was pointed out first by Ando, Nakanishi, and Saito in 1998. ${ }^{55)}$

An important aspect of the Dirac physics is that magnetic fields necessarily cause interactions between upper and lower Dirac cones. In fact, the Dirac formalism allows one to naturally include such "interband effects" of magnetic fields into calculations. In 1970 Fukuyama and Kubo showed, by explicitly performing the calculations, that the large diamagnetism in $\mathrm{Bi}$ and $\mathrm{Bi}_{1-x} \mathrm{Sb}_{x}$ is in fact a consequence of their Dirac nature. ${ }^{50)}$ Intriguingly, due to such interband effects of magnetic fields, the Hall coefficient does not diverge but becomes zero when the carrier density vanishes at the Dirac point, ${ }^{56)}$ which is indeed observed in a topological insulator. ${ }^{57)}$

\subsection{Old proposals for interface Dirac fermions}

It is prudent to mention that already in 1985, the appearance of 2D massless Dirac fermions at a certain interface was anticipated. ${ }^{58,59)}$ Specifically, Volkov and Pankratov discussed that the relative band inversion occurring in $\mathrm{SnTe} / \mathrm{PbTe}$ and in $\mathrm{CdTe} / \mathrm{HgTe}$ would lead to a gapless interface state. ${ }^{58)}$ Also, Fradkin, Dagotto, and Boyanovsky discussed in 1986 that an antiphase domain wall in $\mathrm{PbTe}$ (which creates the atomic arrangements of $\mathrm{Pb}-\mathrm{Te}-\mathbf{P b}-\mathbf{P b}-\mathrm{Te}-\mathrm{Pb}$ or Te-Pb-Te-Te$\mathrm{Pb}-\mathrm{Te}$ ) can be viewed as a parity anomaly in $3+1$ dimension 
and would lead to a gapless interface state. ${ }^{60)}$

What distinguishes the recent discussions of the topological surface states from those old works is the notion of topological protection, which guarantees the stability of the gapless state. For example, SnTe is a band-inverted semiconductor, but its band inversion takes place at an even number of points in the Brillouin zone, which makes 3D SnTe topologically-trivial in the $Z_{2}$ topological classification. ${ }^{35)}$ This means that, even if the interface state is expected to appear at the $\mathrm{SnTe} / \mathrm{PbTe}$ interface as a result of band inversion, its gapless nature is not protected by the $Z_{2}$ topology. (Nevertheless, SnTe was recently found to be a topological crystalline insulator, ${ }^{61,62)}$ and its gapless surface state is actually topologically protected by mirror symmetry, as discussed later.) In the case of the antiphase domain wall in PbTe, detailed symmetry arguments highlighted the difficulty in realizing the parity anomaly. ${ }^{63)}$

\section{Basics of the Topology in Topological Insulators}

\subsection{Berry phase}

The notion of Berry phase ${ }^{64)}$ is important in the discussions of topological phases. Here, I briefly discuss its definition and meaning.

Let $\mathbf{R}(t)$ be a set of time-dependent parameters and consider it as a vector in the parameter space. We write the Hamiltonian of a system specified by the parameter $\mathbf{R}(t)$ as $H[\mathbf{R}(t)]$ and its $n$th eigenstate as $|n, \mathbf{R}(t)\rangle$. The Schrödinger equation for this system is

$$
H[\mathbf{R}(t)]|n, \mathbf{R}(t)\rangle=E_{n}[\mathbf{R}(t)]|n, \mathbf{R}(t)\rangle .
$$

Suppose that $\mathbf{R}$ changes adiabatically from the $t=0$ value $\mathbf{R}_{0}$. The time evolution of the state follows

$$
H[\mathbf{R}(t)]|n, t\rangle=i \hbar \frac{\partial}{\partial t}|n, t\rangle
$$

and the state at time $t$ is given by

$$
|n, t\rangle=\exp \left(\frac{i}{\hbar} \int_{0}^{t} d t^{\prime} L_{n}\left[\mathbf{R}\left(t^{\prime}\right)\right]\right)|n, \mathbf{R}(t)\rangle,
$$

where

$$
L_{n}[\mathbf{R}(t)]=i \hbar \dot{\mathbf{R}}(t) \cdot\left\langle n, \mathbf{R}(t)\left|\nabla_{R}\right| n, \mathbf{R}(t)\right\rangle-E_{n}[\mathbf{R}(t)] .
$$

This can be easily confirmed by putting Eq. (3) into the r.h.s. of Eq. (2). Using $L_{n}[\mathbf{R}(t)]$ given in Eq. (4), one may write the time-dependent state as

$$
\begin{aligned}
|n, t\rangle=\exp \left(-\int_{0}^{t} d t^{\prime} \dot{\mathbf{R}}\left(t^{\prime}\right) \cdot\langle n\right. & \left.\left., \mathbf{R}\left(t^{\prime}\right)\left|\nabla_{R}\right| n, \mathbf{R}\left(t^{\prime}\right)\right\rangle\right)|n, \mathbf{R}(t)\rangle \\
& \times \exp \left(\frac{i}{\hbar} \int_{0}^{t} d t^{\prime} E_{n}\left[\mathbf{R}\left(t^{\prime}\right)\right]\right) .
\end{aligned}
$$

In this expression of $|n, t\rangle$, the first exponential term represents the nontrivial effect of the quantum-mechanical phase accumulated during the time evolution, and the last exponential term is a trivial one called dynamical term.
When $\mathbf{R}$ moves on a closed loop $C$ from $t=0$ and returns to the original position at $t=T$, i.e. $\mathbf{R}(T)=\mathbf{R}_{0}$, the Berry phase $\gamma_{n}[C]$ for this loop $C$ is defined as

$$
\begin{aligned}
\gamma_{n}[C] & \equiv \int_{0}^{T} d t \dot{\mathbf{R}}(t) \cdot i\left\langle n, \mathbf{R}(t)\left|\nabla_{R}\right| n, \mathbf{R}(t)\right\rangle \\
& =\oint_{C} d \mathbf{R} \cdot i\left\langle n, \mathbf{R}\left|\nabla_{R}\right| n, \mathbf{R}\right\rangle \\
& \equiv-\oint_{C} d \mathbf{R} \cdot \mathbf{A}_{n}(\mathbf{R}) \\
& =-\int_{S} d \mathbf{S} \cdot \mathbf{B}_{n}(\mathbf{R})
\end{aligned}
$$

The last equality comes from the Stokes' theorem. Here, we define the Berry connection

$$
\mathbf{A}_{n}(\mathbf{R})=-i\left\langle n, \mathbf{R}\left|\nabla_{R}\right| n, \mathbf{R}\right\rangle,
$$

and its rotation is the Berry curvature

$$
\mathbf{B}_{n}(\mathbf{R})=\nabla_{R} \times \mathbf{A}_{n}(\mathbf{R}) .
$$

From Eqs. (5) and (6), one can see that the Berry phase means the accumulated phase factor of a quantum-mechanical system after it completes a closed path in the parameter space. The Berry connection corresponds to the gauge field defined on that parameter space, similar to the vector potential for electromagnetic fields in real space.

\subsection{TKNN invariant}

The topological invariant defined for the integer quantum Hall system, the TKNN invariant, ${ }^{10}$ ) is closely related to the Berry phase. To see this, we derive the TKNN invariant by calculating the Hall conductivity of a $2 \mathrm{D}$ electron system of size $L \times L$ in perpendicular magnetic fields, where the electric field $E$ and the magnetic field $B$ are applied along the $y$ and $z$ axes, respectively. By treating the effect of the electric field $E$ as a perturbation potential $V=-e E y$, one may use the perturbation theory ${ }^{65)}$ to approximate the perturbed eigenstate $|n\rangle_{E}$ as

$$
|n\rangle_{E}=|n\rangle+\sum_{m(\neq n)} \frac{\langle m|(-e E y)| n\rangle}{E_{n}-E_{m}}|m\rangle+\cdots .
$$

Using this perturbed eigenstate, one may obtain the expectation value of the current density along the $x$ axis, $j_{x}$, in the presence of the $E$ field along the $y$ axis as

$$
\begin{aligned}
\left\langle j_{x}\right\rangle_{E}= & \sum_{n} f\left(E_{n}\right)\left\langle\left. n\right|_{E}\left(\frac{e v_{x}}{L^{2}}\right) \mid n\right\rangle_{E} \\
= & \left\langle j_{x}\right\rangle_{E=0}+\frac{1}{L^{2}} \sum_{n} f\left(E_{n}\right) \sum_{m(\neq n)}\left(\frac{\left\langle n\left|e v_{x}\right| m\right\rangle\langle m|(-e E y)| n\rangle}{E_{n}-E_{m}}\right. \\
& \left.+\frac{\langle n|(-e E y)| m\rangle\left\langle m\left|e v_{x}\right| n\right\rangle}{E_{n}-E_{m}}\right),
\end{aligned}
$$


where $v_{x}$ is the electron velocity along the $x$ direction and $f\left(E_{n}\right)$ is the Fermi distribution function. The Heisenberg equation of motion $\frac{d}{d t} y=v_{y}=\frac{1}{i \hbar}[y, H]$ leads to

$$
\left\langle m\left|v_{y}\right| n\right\rangle=\frac{1}{i \hbar}\left(E_{n}-E_{m}\right)\langle m|y| n\rangle,
$$

which allows one to evaluate

$$
\begin{aligned}
\sigma_{x y}= & \frac{\left\langle j_{x}\right\rangle_{E}}{E} \\
= & -\frac{i \hbar e^{2}}{L^{2}} \sum_{n \neq m} f\left(E_{n}\right) \\
& \times \frac{\left\langle n\left|v_{x}\right| m\right\rangle\left\langle m\left|v_{y}\right| n\right\rangle-\left\langle n\left|v_{y}\right| m\right\rangle\left\langle m\left|v_{x}\right| n\right\rangle}{\left(E_{n}-E_{m}\right)^{2}} .
\end{aligned}
$$

When we consider a system in a periodic potential and its Bloch states $\left|u_{n \mathbf{k}}\right\rangle$ as the eigenstates, the identity

$$
\left\langle u_{m \mathbf{k}^{\prime}}\left|v_{\mu}\right| u_{n \mathbf{k}}\right\rangle=\frac{1}{\hbar}\left(E_{n \mathbf{k}}-E_{m \mathbf{k}^{\prime}}\right)\left\langle u_{m \mathbf{k}^{\prime}}\left|\frac{\partial}{\partial k_{\mu}}\right| u_{n \mathbf{k}}\right\rangle
$$

allows one to rewrite Eq. (15) into the form

$$
\begin{aligned}
\sigma_{x y}= & -\frac{i e^{2}}{\hbar L^{2}} \sum_{\mathbf{k}} \sum_{n \neq m} f\left(E_{n \mathbf{k}}\right) \\
& \times\left(\frac{\partial}{\partial k_{x}}\left\langle u_{n \mathbf{k}} \mid \frac{\partial}{\partial k_{y}} u_{n \mathbf{k}}\right\rangle-\frac{\partial}{\partial k_{y}}\left\langle u_{n \mathbf{k}} \mid \frac{\partial}{\partial k_{x}} u_{n \mathbf{k}}\right\rangle\right) .
\end{aligned}
$$

Since the Berry connection defined in Eq. (10) is written for Bloch states as

$$
\mathbf{a}_{n}(\mathbf{k})=-i\left\langle u_{n \mathbf{k}}\left|\nabla_{k}\right| u_{n \mathbf{k}}\right\rangle=-i\left\langle u_{n \mathbf{k}}\left|\frac{\partial}{\partial \mathbf{k}}\right| u_{n \mathbf{k}}\right\rangle,
$$

the Hall conductivity reduces to

$$
\sigma_{x y}=v \frac{e^{2}}{h}
$$

with

$$
v=\sum_{n} \int_{\mathrm{BZ}} \frac{d^{2} \mathbf{k}}{2 \pi}\left(\frac{\partial a_{n, y}}{\partial k_{x}}-\frac{\partial a_{n, x}}{\partial k_{y}}\right) .
$$

This $v$ can be expressed as $v=\sum_{n} v_{n}$ with $v_{n}$ the contribution from the $n$th band, and one can easily see that $v_{n}$ is related to the Berry phase, namely

$$
\begin{aligned}
v_{n} & =\int_{\mathrm{BZ}} \frac{d^{2} \mathbf{k}}{2 \pi}\left(\frac{\partial a_{n, y}}{\partial k_{x}}-\frac{\partial a_{n, x}}{\partial k_{y}}\right) \\
& =\frac{1}{2 \pi} \oint_{\partial \mathrm{BZ}} d \mathbf{k} \cdot \mathbf{a}_{n}(\mathbf{k}) \\
& =-\frac{1}{2 \pi} \gamma_{n}[\partial \mathrm{BZ}] .
\end{aligned}
$$

Because of the single-valued nature of the wave function, its change in the phase factor after encircling the Brillouin zone boundary $(\partial \mathrm{BZ})$ can only be an integer multiple of $2 \pi$, which means

$$
\gamma_{n}[\partial \mathrm{BZ}]=2 \pi m \quad(m \in Z)
$$

Therefore, $v_{n}$ can only take an integer value, and hence $\sigma_{x y}$ is quantized to integer multiples of $e^{2} / h$. The integer $v$ is called TKNN invariant, and it plays the role of the topological invariant of the quantum Hall system, which is a TRS-breaking TI. It is clear from Eq. (21) that the TKNN invariant becomes nonzero (i.e. the system becomes topological) when the Berry connection $\mathbf{a}_{n}(\mathbf{k})$ is not a single-valued function.

\subsection{Time-reversal operator}

We now consider insulators preserving TRS. The TR operator $\Theta$ for a spin $1 / 2$ particle takes the simple form $\Theta=-i s_{y} K$, where $K$ is the complex conjugate operator. Hereafter, $s_{\mu}$ $(\mu=x, y, z)$ denotes the spin operator given by Pauli matrices. An important property of the TR operator is that

$$
\Theta^{2}=-1 \text {. }
$$

Taking the eigenstates of $s_{z}$ as the basis set $\{|\sigma\rangle\}$, it follows

$$
\begin{aligned}
\langle\psi|\Theta| \phi\rangle & =\sum_{\sigma, \sigma^{\prime}}\langle\psi \mid \sigma\rangle\left\langle\sigma\left|\left(-i s_{y}\right)\right| \sigma^{\prime}\right\rangle\left\langle\sigma^{\prime} \mid \phi^{*}\right\rangle \\
& =-\sum\left\langle\psi \mid \sigma^{*}\right\rangle\left\langle\sigma^{*}\left|i s_{y}\right| \sigma^{\prime}\right\rangle\left\langle\phi \mid\left(\sigma^{\prime}\right)^{*}\right\rangle \\
& =-\sum\left\langle\sigma \mid \psi^{*}\right\rangle\left\langle\left(\sigma^{\prime}\right)^{*}\left|\left(i s_{y}\right)^{\dagger}\right| \sigma\right\rangle\left\langle\phi \mid\left(\sigma^{\prime}\right)^{*}\right\rangle \\
& =-\sum\left\langle\phi \mid\left(\sigma^{\prime}\right)^{*}\right\rangle\left\langle\left(\sigma^{\prime}\right)^{*}\left|\left(-i s_{y}\right)\right| \sigma\right\rangle\left\langle\sigma \mid \psi^{*}\right\rangle \\
& =-\langle\phi|\Theta| \psi\rangle .
\end{aligned}
$$

Similarly, one obtains

$$
\begin{aligned}
& \langle\Theta \psi \mid \Theta \phi\rangle=\left(\sum_{\sigma}\left\langle\sigma^{*} \mid \psi\right\rangle\langle\sigma|\left(+i s_{y}\right)\right)\left(\sum_{\sigma^{\prime}}\left(-i s_{y}\right)\left|\sigma^{\prime}\right\rangle\left\langle\sigma^{\prime} \mid \phi^{*}\right\rangle\right) \\
& =\sum_{\sigma, \sigma^{\prime}}\left\langle\phi \mid\left(\sigma^{\prime}\right)^{*}\right\rangle\left\langle\sigma \mid \sigma^{\prime}\right\rangle\left\langle\sigma^{*} \mid \psi\right\rangle \\
& =\sum\left\langle\phi \mid\left(\sigma^{\prime}\right)^{*}\right\rangle\left\langle\left(\sigma^{\prime}\right)^{*} \mid \sigma^{*}\right\rangle\left\langle\sigma^{*} \mid \psi\right\rangle \\
& =\langle\phi \mid \psi\rangle \text {. }
\end{aligned}
$$

Also, for arbitrary linear operator $A$

$$
\left\langle\Theta \psi\left|\Theta A \Theta^{-1}\right| \Theta \phi\right\rangle=\left\langle\phi\left|A^{\dagger}\right| \psi\right\rangle .
$$

\subsection{TRS and Bloch Hamiltonian}

Let $\mathcal{H}$ be the total Hamiltonian of a periodic system:

$$
\mathcal{H}\left|\psi_{n \mathbf{k}}\right\rangle=E_{n \mathbf{k}}\left|\psi_{n \mathbf{k}}\right\rangle .
$$

Bloch's theorem leads to the separation of $\psi_{n \mathbf{k}}$ into

$$
\left|\psi_{n \mathbf{k}}\right\rangle=e^{i \mathbf{k} \cdot \mathbf{r}}\left|u_{n \mathbf{k}}\right\rangle,
$$

where $\left|u_{n \mathbf{k}}\right\rangle$ is the cell-periodic eigenstate of the Bloch Hamiltonian

$$
H(\mathbf{k})=e^{-i \mathbf{k} \cdot \mathbf{r}} \mathcal{H} e^{i \mathbf{k} \cdot \mathbf{r}},
$$

and $\left|u_{n \mathbf{k}}\right\rangle$ satisfies the reduced Schrödinger equation

$$
H(\mathbf{k})\left|u_{n \mathbf{k}}\right\rangle=E_{n \mathbf{k}}\left|u_{n \mathbf{k}}\right\rangle .
$$




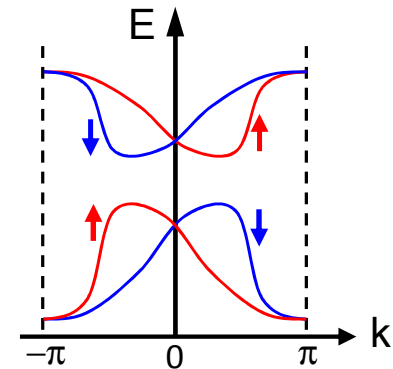

Fig. 4. (Color online) Kramers pairs of bands. Note that each pair of bands are degenerate at the TR-invariant momentum (TRIM) where $+\mathbf{k}$ becomes equivalent to $-\mathbf{k}$ due to the periodicity of the Brillouin zone. In this figure, there are two TRIMs, $k=0$ and $k=\pi$ (which is equivalent to $k=-\pi$ ). The lifting of degeneracy at $k$ values other than 0 and $\pi$ comes from spin-orbit coupling.

It is important to note that when $\mathcal{H}$ preserves TRS, i.e. $[\mathcal{H}, \Theta]=0$, then $H(\mathbf{k})$ satisfies

$$
H(-\mathbf{k})=\Theta H(\mathbf{k}) \Theta^{-1} .
$$

This identity means that the energy bands of a TR symmetric system come in pairs (i.e. $+\mathbf{k}$ state and $-\mathbf{k}$ state are at the same energy), and they are called Kramers pairs (Fig. 4). Naturally, Kramers pairs are degenerate at the TR-invariant momentum (TRIM) where $+\mathbf{k}$ becomes equivalent to $-\mathbf{k}$ due to the periodicity of the BZ (Fig. 5).

Now we discuss a matrix representation of the TR operator in the Bloch wave function basis. A convenient matrix is

$$
w_{\alpha \beta}(\mathbf{k})=\left\langle u_{\alpha,-\mathbf{k}}|\Theta| u_{\beta, \mathbf{k}}\right\rangle,
$$

where $\alpha$ and $\beta$ are band indices. This matrix relates the two Bloch states $\left|u_{\alpha,-\mathbf{k}}\right\rangle$ and $\left|u_{\beta, \mathbf{k}}\right\rangle$ via

$$
\left|u_{\alpha,-\mathbf{k}}\right\rangle=\sum_{\beta} w_{\alpha \beta}^{*}(\mathbf{k}) \Theta\left|u_{\beta, \mathbf{k}}\right\rangle .
$$

One can easily confirm that $w_{\alpha \beta}(\mathbf{k})$ is a unitary matrix, i.e.

$$
\sum_{\alpha} w_{\gamma \alpha}^{\dagger}(\mathbf{k}) w_{\alpha \beta}(\mathbf{k})=\left\langle u_{\beta, \mathbf{k}} \mid u_{\gamma, \mathbf{k}}\right\rangle=\delta_{\beta \gamma},
$$

and it has the following property:

$$
w_{\beta \alpha}(-\mathbf{k})=-w_{\alpha \beta}(\mathbf{k}) .
$$

This equation means that at a TRIM $\Lambda$, the $w$ matrix becomes antisymmetric, i.e.

$$
w_{\beta \alpha}(\mathbf{\Lambda})=-w_{\alpha \beta}(\boldsymbol{\Lambda}) .
$$

For example, if there are only two occupied bands, $w_{\alpha \beta}$ becomes a $2 \times 2$ matrix and at $\boldsymbol{\Lambda}$ it can be explicitly written as

$$
w(\mathbf{\Lambda})=\left(\begin{array}{cc}
0 & w_{12}(\mathbf{\Lambda}) \\
-w_{12}(\mathbf{\Lambda}) & 0
\end{array}\right)=w_{12}(\mathbf{\Lambda})\left(\begin{array}{cc}
0 & 1 \\
-1 & 0
\end{array}\right) .
$$

Another convenient matrix to consider for a TR symmetric
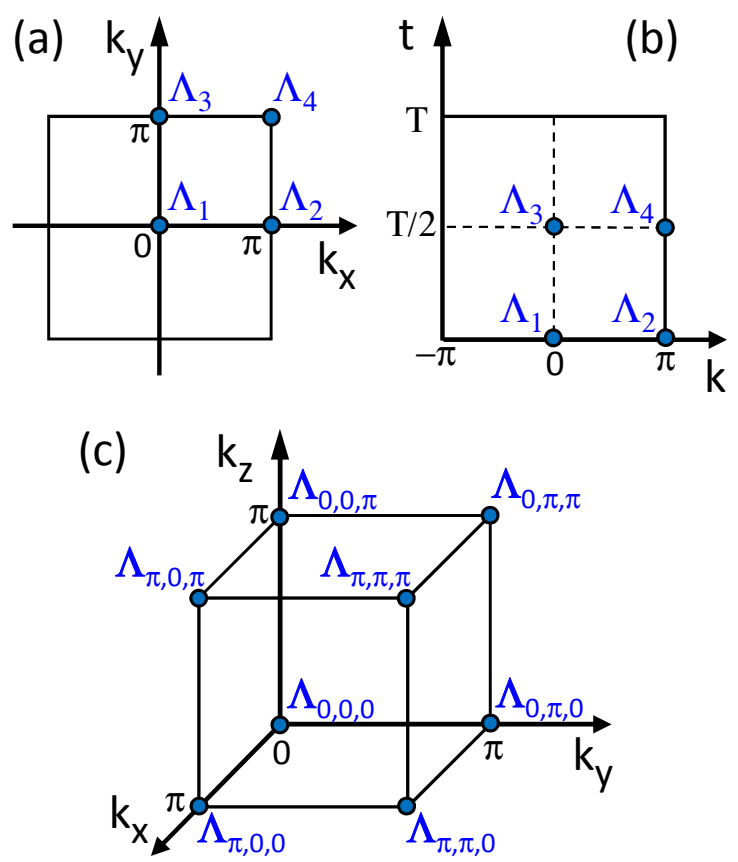

Fig. 5. (Color online) Time-reversal-invariant momenta (TRIMs). (a) There are 4 TRIMs for a 2D BZ; this figure shows the case of a square BZ. (b) The TRIMs for a TR-symmetric 1D system considered in Sec. 3.5. One can see that identifying $t \rightarrow k_{y}$ maps the periodic $(k, t)$ space to the ordinary 2D BZ shown in (a). (c) There are 8 TRIMs for a 3D BZ; this figure exemplifies the case of a cubic BZ.

system is the $U(2)$ Berry connection matrix

$$
\mathbf{a}_{\alpha \beta}(\mathbf{k})=-i\left\langle u_{\alpha, \mathbf{k}}\left|\nabla_{\mathbf{k}}\right| u_{\beta, \mathbf{k}}\right\rangle .
$$

Notice that $\mathbf{a}$ is actually a set of three matrices. By using Eqs. (25) and (33), it can be shown that $\mathbf{a}_{\alpha \beta}(\mathbf{k})$ and $\mathbf{a}_{\alpha \beta}(-\mathbf{k})$ are related by

$$
\mathbf{a}(-\mathbf{k})=w(\mathbf{k}) \mathbf{a}^{*}(\mathbf{k}) w^{\dagger}(\mathbf{k})+i w(\mathbf{k}) \nabla_{\mathbf{k}} w^{\dagger}(\mathbf{k}),
$$

and taking the trace gives

$$
\operatorname{tr}[\mathbf{a}(-\mathbf{k})]=\operatorname{tr}\left[\mathbf{a}^{*}(\mathbf{k})\right]+i \operatorname{tr}\left[w(\mathbf{k}) \nabla_{\mathbf{k}} w^{\dagger}(\mathbf{k})\right] .
$$

Noticing that $\operatorname{tr}[\mathbf{a}]=\operatorname{tr}\left[\mathbf{a}^{*}\right]$ (because $\mathbf{a}_{\beta \alpha}=\mathbf{a}^{*}{ }_{\alpha \beta}$ ) and that $w \nabla w^{\dagger}=-(\nabla w) w^{\dagger}$ (because $w w^{\dagger}=1$ ), the replacement $-\mathbf{k} \rightarrow$ $\mathbf{k}$ leads to

$$
\operatorname{tr}[\mathbf{a}(\mathbf{k})]=\operatorname{tr}[\mathbf{a}(-\mathbf{k})]+i \operatorname{tr}\left[w^{\dagger}(\mathbf{k}) \nabla_{\mathbf{k}} w(\mathbf{k})\right] .
$$

This relation becomes important in the calculation of the $Z_{2}$ topological invariant.

\section{5 $\quad Z_{2}$ time-reversal polarization}

In order to pedagogically derive the topological invariant to characterize 2D electron systems preserving TRS, we follow Fu and Kane ${ }^{66)}$ and consider a 1D system with length $L$ and lattice constant $a=1$. We only consider two bands that form a Kramers pair, and denote their Bloch wave functions as 
$\left|u_{1}(k)\right\rangle$ and $\left|u_{2}(k)\right\rangle$. Assume that the band parameters change with time and return to the original values at $t=T$. Furthermore, we consider the situation when the 1D Hamiltonian satisfies the following conditions:

$$
\begin{aligned}
& H[t+T]=H[t] \\
& H[-t]=\Theta H[t] \Theta^{-1} .
\end{aligned}
$$

From the modern theory of ferroelectricity, ${ }^{67)}$ it is known that charge polarization $P_{\rho}$ can be calculated by integrating the Berry connection of the occupied states over the BZ. In the present case of the $1 \mathrm{D}$ two-band system, $P_{\rho}$ may be written as

$$
P_{\rho}=\int_{-\pi}^{\pi} \frac{d k}{2 \pi} A(k),
$$

where

$$
\begin{aligned}
A(k) & =-i\left\langle u_{1}(k)\left|\nabla_{k}\right| u_{1}(k)\right\rangle-i\left\langle u_{2}(k)\left|\nabla_{k}\right| u_{2}(k)\right\rangle \\
& =a_{11}(k)+a_{22}(k) \\
& =\operatorname{tr}[a] .
\end{aligned}
$$

The contribution from each band may be called partial polarization defined by

$$
P_{i}=\int_{-\pi}^{\pi} \frac{d k}{2 \pi} a_{i i}(k) \quad(i=1,2),
$$

which gives the relation $P_{\rho}=P_{1}+P_{2}$. The time-reversal polarization is defined by

$$
P_{\theta}=P_{1}-P_{2}=2 P_{1}-P_{\rho} .
$$

Intuitively, $P_{\theta}$ gives the difference in charge polarization between spin-up and spin-down bands, since $\left|u_{1}(k)\right\rangle$ and $\left|u_{2}(k)\right\rangle$ form a Kramers pair.

From Eqs. (42) and (43), one can see that the system is TR symmetric at $t=0$ and $t=T / 2$. At these times, the Kramers degeneracy must be observed at any $k$, which dictates that the time-reversed version of $\left|u_{2}(k)\right\rangle$, i.e. $\Theta\left|u_{2}(k)\right\rangle$, is equal to $\left|u_{1}(-k)\right\rangle$ except for a phase factor. Hence, at $t=0$ and $t=T / 2$,

$$
\begin{aligned}
& \Theta\left|u_{2}(k)\right\rangle=e^{-i \chi(k)}\left|u_{1}(-k)\right\rangle \\
& \Theta\left|u_{1}(k)\right\rangle=-e^{-i \chi(-k)}\left|u_{2}(-k)\right\rangle .
\end{aligned}
$$

Using these relations, the $w$ matrix can be shown to become

$$
w(k)=\left(\begin{array}{cc}
0 & e^{-i \chi(k)} \\
-e^{-i \chi(-k)} & 0
\end{array}\right) .
$$

Now we calculate $P_{1}$ at the TR symmetric times. First, using Eq. (50) one may obtain

$$
a_{11}(-k)=a_{22}(k)-\frac{\partial}{\partial k} \chi(k),
$$

which leads to

$$
P_{1}=\frac{1}{2 \pi}\left(\int_{0}^{\pi} d k a_{11}(k)+\int_{-\pi}^{0} d k a_{11}(k)\right)
$$

$$
\begin{aligned}
& =\frac{1}{2 \pi} \int_{0}^{\pi} d k\left(a_{11}(k)+a_{22}(k)-\frac{\partial}{\partial k} \chi(k)\right) \\
& =\int_{0}^{\pi} \frac{d k}{2 \pi} A(k)-\frac{1}{2 \pi}[\chi(\pi)-\chi(0)] .
\end{aligned}
$$

Since $w_{12}(k)=e^{-i \chi(k)}$ from Eq. (51), $\chi(k)$ can be written as

$$
\chi(k)=i \log w_{12}(k)
$$

and Eq. (53) reduces to

$$
P_{1}=\int_{0}^{\pi} \frac{d k}{2 \pi} A(k)-\frac{i}{2 \pi} \log \frac{w_{12}(\pi)}{w_{12}(0)} .
$$

This expression leads to

$$
P_{\theta}=2 P_{1}-P_{\rho}=\int_{0}^{\pi} \frac{d k}{2 \pi}[A(k)-A(-k)]-\frac{i}{\pi} \log \frac{w_{12}(\pi)}{w_{12}(0)} .
$$

Noticing $A(k)=\operatorname{tr}[a(k)]$ and using Eq. (41), one obtains

$$
\begin{aligned}
P_{\theta} & =\int_{0}^{\pi} \frac{d k}{2 \pi} i \operatorname{tr}\left[w^{\dagger}(k) \frac{\partial}{\partial k} w(k)\right]-\frac{i}{\pi} \log \frac{w_{12}(\pi)}{w_{12}(0)} \\
& =i \int_{0}^{\pi} \frac{d k}{2 \pi} \frac{\partial}{\partial k} \log (\operatorname{det}[w(k)])-\frac{i}{\pi} \log \frac{w_{12}(\pi)}{w_{12}(0)} \\
& =\frac{i}{\pi} \cdot \frac{1}{2} \log \frac{\operatorname{det}[w(\pi)]}{\operatorname{det}[w(0)]}-\frac{i}{\pi} \log \frac{w_{12}(\pi)}{w_{12}(0)}
\end{aligned}
$$

Since $\operatorname{det}[w]=w_{12}^{2}$ in the present case, Eq. (57) reduces to

$$
P_{\theta}=\frac{1}{i \pi} \log \left(\frac{\sqrt{w_{12}(0)^{2}}}{w_{12}(0)} \cdot \frac{w_{12}(\pi)}{\sqrt{w_{12}(\pi)^{2}}}\right) .
$$

The argument of this $\log$ is +1 or -1 . Since $\log (-1)=i \pi$ if the angle in the complex plane is restricted from 0 to $2 \pi$, one can see that $P_{\theta}$ is 0 or $1(\bmod 2)$. Physically, the two values of $P_{\theta}$ corresponds to two different polarization states which the present 1D system can take at $t=0$ and $t=T / 2$.

We now consider the change of $P_{\theta}$ from $t=0$ to $T / 2$. The wave function $\left|u_{n}(k, t)\right\rangle$ can be viewed as a map from the 2D phase space $(k, t)$, which forms a torus due to the periodic boundary conditions, to the Hilbert space. This Hilbert space can be classified into two groups depending on the difference in $P_{\theta}$ between $t=0$ and $t=T / 2$,

$$
\Delta=P_{\theta}(T / 2)-P_{\theta}(0) \text {. }
$$

This $\Delta$ is specified only in $\bmod 2$, so it gives a $Z_{2}$ topological invariant to characterize the Hilbert space. Intuitively, when $P_{\theta}$ changes between $t=0$ to $T / 2$, the Hilbert space is "twisted" and $\Delta=1$, while the Hilbert space is trivial $(\Delta=0)$ when there is no change in $P_{\theta}$. Using Eq. (58), $\Delta$ can be given in terms of

$$
(-1)^{\Delta}=\prod_{i=1}^{4} \frac{w_{12}\left(\Lambda_{i}\right)}{\sqrt{w_{12}\left(\Lambda_{i}\right)^{2}}}
$$

where $\Lambda_{1}=(k, t)=(0,0), \Lambda_{2}=(\pi, 0), \Lambda_{3}=(0, T / 2)$, and $\Lambda_{4}=$ $(\pi, T / 2)$ [see Fig. 5(b)]. The physical consequence of a cycle with $\Delta=1$ is spin pumping from one end of the $1 \mathrm{D}$ system to the other. ${ }^{66)}$ 


\subsection{General formula of the $Z_{2}$ invariant}

Extension of the above argument to a multiband system is not very difficult. The Hamiltonian still satisfies Eqs. (42) and (43), and in the following we take $T=2 \pi$ for simplicity. Consider that $2 N$ bands are occupied and forming $N$ Kramers pairs. For each Kramers pair $n$, at the TR symmetric times $t=0$ and $\pi$ the wave functions are related by

$$
\begin{aligned}
& \Theta\left|u_{2}^{n}(k)\right\rangle=e^{-i \chi_{n}(k)}\left|u_{1}^{n}(-k)\right\rangle \\
& \Theta\left|u_{1}^{n}(k)\right\rangle=-e^{-i \chi_{n}(-k)}\left|u_{2}^{n}(-k)\right\rangle,
\end{aligned}
$$

and the $w$ matrix is given by

$$
w(k)=\left(\begin{array}{ccccc}
0 & e^{-i \chi_{1}(k)} & 0 & 0 & \ldots \\
-e^{-i \chi_{1}(-k)} & 0 & 0 & 0 & \ldots \\
0 & 0 & 0 & e^{-i \chi_{2}(k)} & \ldots \\
0 & 0 & -e^{-i \chi_{2}(-k)} & 0 & \ldots \\
\vdots & \vdots & \vdots & \vdots & \ddots
\end{array}\right) .
$$

Hence, at $t=0$ and $\pi, w(0)$ and $w(\pi)$ become antisymmetric and we have

$$
\begin{aligned}
w_{12}\left(\Lambda_{i}\right) w_{34}\left(\Lambda_{i}\right) \ldots w_{2 N-1,2 N}\left(\Lambda_{i}\right) & =e^{-i \sum_{n=1}^{N} \chi_{n}\left(\Lambda_{i}\right)} \\
& =\operatorname{Pf}\left[w\left(\Lambda_{i}\right)\right]
\end{aligned}
$$

Note that in the above equation, $w$ is viewed as a function of $k$ and $t$, and we used the formula for the Pfaffian of a $2 N \times 2 N$ skew-symmetric matrix with $2 \times 2$ blocks on the diagonal. In general, Pfaffian is defined for an antisymmetric matrix and is related to the determinant by

$$
\operatorname{Pf}[A]^{2}=\operatorname{det}[A] .
$$

It is straightforward to extend the previous calculations for the TR symmetric times $t=0$ and $\pi$ to obtain

$$
\begin{aligned}
P_{1} & =\int_{0}^{\pi} \frac{d k}{2 \pi} A(k)-\frac{1}{2 \pi} \sum_{n=1}^{N}\left[\chi_{n}(\pi)-\chi_{n}(0)\right] \\
& =\int_{0}^{\pi} \frac{d k}{2 \pi} A(k)-\frac{i}{2 \pi} \log \left(\frac{\operatorname{Pf}[w(\pi)]}{\operatorname{Pf}[w(0)]}\right),
\end{aligned}
$$

which in turn gives the TR polarization

$$
P_{\theta}=\frac{1}{i \pi} \log \left(\frac{\sqrt{\operatorname{det}[w(0)]}}{\operatorname{Pf}[w(0)]} \cdot \frac{\operatorname{Pf}[w(\pi)]}{\sqrt{\operatorname{det}[w(\pi)]}}\right) .
$$

Therefore, the $Z_{2}$ topological invariant $v$ is given by

$$
(-1)^{v}=\prod_{i=1}^{4} \frac{\operatorname{Pf}\left[w\left(\Lambda_{i}\right)\right]}{\sqrt{\operatorname{det}\left[w\left(\Lambda_{i}\right)\right]}},
$$

which classifies the Hilbert space into "twisted" $(v=1)$ and trivial $(v=0)$ ones.

By reinterpreting the periodic 2D phase space $(k, t)$, which forms a torus, as the 2D Brillouin zone $\left(k_{x}, k_{y}\right)$, the above theory provides a $Z_{2}$ topological classification of $2 \mathrm{D}$ TRinvariant insulators with $2 \mathrm{~N}$ occupied bands.

\subsection{Extension to $3 D$ systems}

Based on a general homotopy argument, Moore and Balents showed ${ }^{32)}$ that there are four $Z_{2}$ invariants for $3 \mathrm{D}$ systems. While the construction of the homotopy was involved, the physical origin of the four invariants can be easily understood. For simplicity, consider a cubic system and take the lattice constant $a=1$. In the 3D BZ of this system, there are eight TRIMs denoted as $\Lambda_{0,0,0}, \Lambda_{\pi, 0,0}, \Lambda_{0, \pi, 0}, \Lambda_{0,0, \pi}, \Lambda_{\pi, 0, \pi}$, $\Lambda_{0, \pi, \pi}, \Lambda_{\pi, \pi, 0}$, and $\Lambda_{\pi, \pi, \pi}$ [see Fig. 5(c)]. At these points in the BZ, the Bloch Hamiltonian becomes TR symmetric, i.e. $\Theta H\left(\Lambda_{i}\right) \Theta^{-1}=H\left(\Lambda_{i}\right)$, and each Kramers pair of bands become degenerate.

Notice that the six planes in the 3D BZ, $x=0, x= \pm \pi$, $y=0, y= \pm \pi, z=0$, and $z= \pm \pi$ possess the symmetries of the $2 \mathrm{D} \mathrm{BZ}$, and therefore they each have a $Z_{2}$ invariant. The six invariants may be denoted as $x_{0}, x_{1}, y_{0}, y_{1}, z_{0}$, and $z_{1}$, but those six invariants are not all independent. ${ }^{32)}$ This is because the products $x_{0} x_{1}, y_{0} y_{1}$, and $z_{0} z_{1}$ are redundant, which stems from the fact that those three products involve $\left.\operatorname{Pf}\left[w\left(\Lambda_{i}\right)\right] / \sqrt{\operatorname{det}\left[w\left(\Lambda_{i}\right)\right.}\right]$ from all eight TRIMs and hence are the same. This means that there are two constraining relations $x_{0} x_{1}=y_{0} y_{1}=z_{0} z_{1}$, which dictates that only four invariants can be independently determined in a 3D system.

The concrete construction of the four $Z_{2}$ invariants were given by $\mathrm{Fu}$ and Kane. ${ }^{33)}$ For each TRIM $\Lambda_{i}$, we define

$$
\delta\left(\Lambda_{i}\right) \equiv \frac{\operatorname{Pf}\left[w\left(\Lambda_{i}\right)\right]}{\sqrt{\operatorname{det}\left[w\left(\Lambda_{i}\right)\right]}} .
$$

Using this $\delta\left(\Lambda_{i}\right)$, the four $Z_{2}$ invariants $v_{0}, v_{1}, v_{2}, v_{3}$ are defined as

$$
\begin{aligned}
& (-1)^{v_{0}}=\prod_{n_{j}=0, \pi} \delta\left(\Lambda_{n_{1}, n_{2}, n_{3}}\right) \\
& (-1)^{v_{i}}=\prod_{n_{j \neq i}=0, \pi ; n_{i}=\pi} \delta\left(\Lambda_{n_{1}, n_{2}, n_{3}}\right) \quad(i=1,2,3) .
\end{aligned}
$$

The invariant $v_{0}$ is given by a product of all eight $\delta\left(\Lambda_{i}\right)$ 's, so it is unique to a $3 \mathrm{D}$ system. On the other hand, other $v_{i}$ 's are a product of four $\delta\left(\Lambda_{i}\right)$ 's and is similar to the $Z_{2}$ invariant in the $2 \mathrm{D}$ case. For example,

$$
(-1)^{\nu_{3}}=\delta\left(\Lambda_{0,0, \pi}\right) \delta\left(\Lambda_{\pi, 0, \pi}\right) \delta\left(\Lambda_{0, \pi, \pi}\right) \delta\left(\Lambda_{\pi, \pi, \pi}\right)
$$

corresponds to the $Z_{2}$ invariant on the $z=\pi$ plane, which can be seen by considering the TR polarization defined on this plane, $P_{\theta}(y=0)_{z=\pi}=\frac{1}{i \pi} \log \left[\delta\left(\Lambda_{0,0, \pi}\right) \delta\left(\Lambda_{\pi, 0, \pi}\right)\right]$ and $P_{\theta}(y=$ $\pi)_{z=\pi}=\frac{1}{i \pi} \log \left[\delta\left(\Lambda_{0, \pi, \pi}\right) \delta\left(\Lambda_{\pi, \pi, \pi}\right)\right]$. When the TR polarization changes between $y=0$ and $y=\pi$ [i.e. $P_{\theta}(y=0)_{z=\pi}$ and $P_{\theta}(y=$ $\pi)_{z=\pi}$ are different], then the $Z_{2}$ topology is nontrivial and $v_{3}$ becomes 1 .

The physical consequence of a nontrivial $Z_{2}$ invariant is the appearance of topologically-protected surface states. This is graphically shown in Fig. 6, in which topologically trivial and nontrivial surface states are compared. In the nontrivial case, the Kramers pairs in the surface state "switch partners", and as a result, the surface state is guaranteed to cross any Fermi 


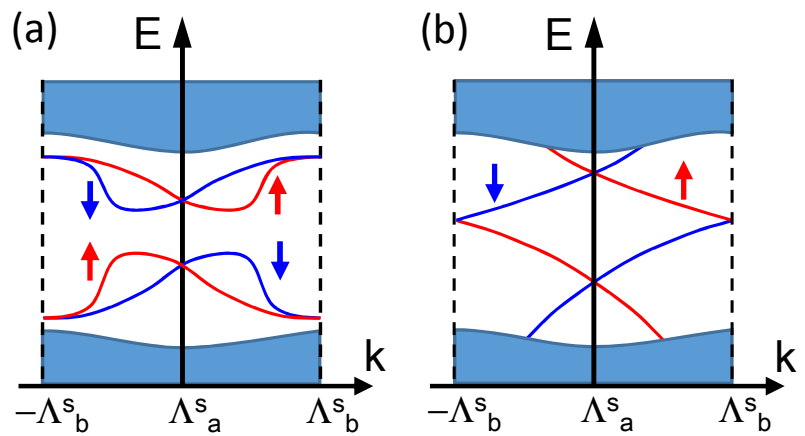

Fig. 6. (Color online) Schematic pictures of the surface states between two surface TRIMs, $\Lambda_{a}^{s}$ and $\Lambda_{b}^{s}$, for (a) topologically trivial and (b) topologically nontrivial cases. In the latter, the "switch-partner" characteristic is a reflection of the change in the TR polarization (see text). The shaded regions represent the bulk continuum states. To enhance the visibility of the Kramers degeneracy, surface state dispersions are shown from $-\Lambda_{b}^{s}$ to $\Lambda_{b}^{s}$ through $\Lambda_{a}^{s}$.

energy inside the bulk gap. This switch-partner characteristic is a reflection of the change in the TR polarization discussed above.

It is customary to write the combination of the four invariants in the form $\left(v_{0} ; v_{1} v_{2} v_{3}\right)$, because $\left(v_{1} v_{2} v_{3}\right)$ can be interpreted as Miller indices to specify the direction of vector $\Lambda_{i}$ in the reciprocal space. A 3D TI is called "strong" when $v_{0}=$ 1 , while it is called "weak" when $v_{0}=0$ and $v_{i}=1$ for some $i$ $=1,2,3$.

\subsection{Topological insulators with inversion symmetry}

Define the space inversion operator $\Pi$ as

$$
\Pi|\mathbf{x}, \sigma\rangle=|-\mathbf{x}, \sigma\rangle,
$$

where $\mathbf{x}$ is the spatial coordinate and $\sigma$ signifies the spin. One can easily see that

$$
\Pi^{2}=1
$$

which means that the eigenvalue of the $\Pi$ operator is \pm 1 . It is easy to see that $\Pi$ operates on the wave function in momentum representation as $\Pi|\mathbf{k}, \sigma\rangle=|-\mathbf{k}, \sigma\rangle$, and hence Hamiltonian $H=\sum_{\mathbf{k}, \sigma, \sigma^{\prime}}|\mathbf{k}, \sigma\rangle H_{\sigma, \sigma^{\prime}}(\mathbf{k})\left\langle\mathbf{k}, \sigma^{\prime}\right|$ transforms as

$$
\begin{aligned}
\Pi H \Pi^{-1} & =\sum_{\mathbf{k}, \sigma, \sigma^{\prime}}|-\mathbf{k}, \sigma\rangle H_{\sigma, \sigma^{\prime}}(\mathbf{k})\left\langle-\mathbf{k}, \sigma^{\prime}\right| \\
& =\sum_{\mathbf{k}, \sigma, \sigma^{\prime}}|\mathbf{k}, \sigma\rangle H_{\sigma, \sigma^{\prime}}(-\mathbf{k})\left\langle\mathbf{k}, \sigma^{\prime}\right| .
\end{aligned}
$$

When $H$ satisfies $H(-\mathbf{k})=H(\mathbf{k})$, it also satisfies

$$
\Pi H \Pi^{-1}=H
$$

and hence the system preserves inversion symmetry (IS). Our aim is to express the $Z_{2}$ invariant using the eigenvalues of $\Pi$, namely, the parity.

We have previously defined the $U(2)$ Berry connection matrix $\mathbf{a}_{\alpha \beta}(\mathbf{k})$ in Eq. (38) and calculated its trace, $\operatorname{tr}[\mathbf{a}(\mathbf{k})]$, in Eq.
(41). Now we denote this trace as $\mathbf{a}^{c}(\mathbf{k})$ and define

$$
F(\mathbf{k})=\nabla_{k} \times \mathbf{a}^{c}(\mathbf{k})
$$

which is the Berry curvature of $\mathbf{a}^{c}$. The two symmetries dictate that this $F(\mathbf{k})$ has the following properties: ${ }^{68)}$

$$
\begin{aligned}
\mathrm{TRS} & \rightarrow F(-\mathbf{k})=-F(\mathbf{k}) \\
\mathrm{IS} & \rightarrow F(-\mathbf{k})=+F(\mathbf{k}) .
\end{aligned}
$$

Therefore, the Berry curvature $F(\mathbf{k})=0$ for any $\mathbf{k}$, and hence one can always chose a gauge which makes $\mathbf{a}^{c}(\mathbf{k})=0$.

To actually obtain a gauge to set $\mathbf{a}^{c}(\mathbf{k})=0$ for any $\mathbf{k}$, we consider the matrix

$$
v_{\alpha \beta}(\mathbf{k}) \equiv\left\langle u_{\alpha \mathbf{k}}|\Pi \Theta| u_{\beta \mathbf{k}}\right\rangle .
$$

One can see that this $v$-matrix is antisymmetric $\left(v_{\alpha \beta}=-v_{\beta \alpha}\right)$ and unitary $\left(\sum_{\beta} v_{\alpha \beta} v_{\gamma \beta}^{*}=\delta_{\alpha \gamma}\right)$. Furthermore, $v$ is related to $\mathbf{a}^{c}(\mathbf{k})$ via

$$
\frac{i}{2} \operatorname{tr}\left[v^{\dagger} \nabla_{k} v\right]=\mathbf{a}^{c}(\mathbf{k})
$$

which can be verified by calculations similar to those employed for deriving Eq. (41). Since $\operatorname{tr}\left[v^{\dagger} \nabla_{k} v\right]=\nabla_{k} \operatorname{tr}[\log v]=$ $\nabla_{k} \log (\operatorname{det}[v]),{ }^{35)}$ one can see

$$
\mathbf{a}^{c}(\mathbf{k})=\frac{i \nabla_{k}}{2} \log (\operatorname{det}[v(\mathbf{k})])=i \nabla_{k} \log \operatorname{Pf}[v(\mathbf{k})] .
$$

To set $\mathbf{a}^{c}(\mathbf{k})=0$, all we have to do is to adjust the phase of $\left|u_{\alpha \mathbf{k}}\right\rangle$ to make $\operatorname{Pf}[v(\mathbf{k})]=1$.

Now we calculate the $w$-matrix of an inversion symmetric system. Let $\xi_{\alpha}\left(\Lambda_{i}\right)= \pm 1$ be the eigenvalue of $\Pi$ for band $\alpha$ at $\operatorname{TRIM} \Lambda_{i}$, namely, $\Pi\left|u_{\alpha}\left(\Lambda_{i}\right)\right\rangle=\xi_{\alpha}\left(\Lambda_{i}\right)\left|u_{\alpha}\left(\Lambda_{i}\right)\right\rangle$. Remembering $\Pi^{2}=1$, one may calculate

$$
\begin{aligned}
w_{\alpha \beta}\left(\Lambda_{i}\right) & =\left\langle u_{\alpha}\left(-\Lambda_{i}\right)|\Pi \Pi \Theta| u_{\beta}\left(\Lambda_{i}\right)\right\rangle \\
& =\xi_{\alpha}\left(\Lambda_{i}\right)\left\langle u_{\alpha}\left(\Lambda_{i}\right)|\Pi \Theta| u_{\beta}\left(\Lambda_{i}\right)\right\rangle \\
& =\xi_{\alpha}\left(\Lambda_{i}\right) v_{\alpha \beta}\left(\Lambda_{i}\right) .
\end{aligned}
$$

Since $w_{\alpha \beta}$ and $v_{\alpha \beta}$ are antisymmetric, $\xi_{\alpha}=\xi_{\beta}$ when $w_{\alpha \beta}=$ $-w_{\beta \alpha} \neq 0$. Such a non-zero $w_{\alpha \beta}$ is obtained only when the bands $\alpha$ and $\beta$ form a Kramers pair. Hence, if the bands $\alpha$ and $\beta$ are the $n$th Kramers pair in the total of $2 N$ bands, we may write $\xi_{\alpha}=\xi_{\beta} \equiv \xi_{2 n}$. Remembering that $w_{\alpha \beta}$ has the peculiar skew-symmetric from of Eq. (63), one can see

$$
\operatorname{Pf}\left[w\left(\Lambda_{i}\right)\right]=\operatorname{Pf}\left[v\left(\Lambda_{i}\right)\right] \prod_{n=1}^{N} \xi_{2 n}\left(\Lambda_{i}\right)
$$

Since we have chosen the gauge to give $\operatorname{Pf}[v(\mathbf{k})]=1$, we finally obtain

$$
\delta\left(\Lambda_{i}\right)=\frac{\operatorname{Pf}\left[w\left(\Lambda_{i}\right)\right]}{\sqrt{\operatorname{det}\left[w\left(\Lambda_{i}\right)\right]}}=\prod_{n=1}^{N} \xi_{2 n}\left(\Lambda_{i}\right) .
$$

This means that the $Z_{2}$ invariant can be calculated simply by the parity eigenvalues $\xi_{2 n}$ at TRIMs $\Lambda_{i}$. For example, for a 2D system with $2 N$ bands preserving IS and TRS, the $Z_{2}$ invariant 
$v$ is given by

$$
(-1)^{v}=\prod_{i=1}^{4} \delta\left(\Lambda_{i}\right)=\prod_{i=1}^{4} \prod_{n=1}^{N} \xi_{2 n}\left(\Lambda_{i}\right) .
$$

This simplification of the calculation of the $Z_{2}$ index, accomplished by $\mathrm{Fu}$ and Kane, ${ }^{35)}$ greatly facilitated the theoretical predictions of candidate TI materials based on $a b$ initio band calculations.

\subsection{BHZ model}

Now that we have learned how the topology of a TRinvariant insulator can be specified, let us consider a concrete example that gives rise to a topologically nontrivial state. As mentioned in Sec. 2.2, the first theoretical model proposed in this context was the Kane-Mele model based on graphene, ${ }^{25)}$ but it was the $\mathrm{BHZ}$ model $^{30)}$ that actually led to the experimental discovery of the first 2D TI material. In this section, we discuss the BHZ model within the framework developed by $\mathrm{Fu}$ and $\mathrm{Kane}^{35)}$ to make its $Z_{2}$ topology transparent.

The BHZ model is motivated by $\mathrm{CdTe} / \mathrm{HgTe} / \mathrm{CdTe}$ quantum well, where the $s$ - and $p$-orbital bands invert at $\mathbf{k}=0$ as a function of the well width. In this quantum-well structure, two $s$-orbital states and two $p_{m=3 / 2}$-orbital states appear near the Fermi energy. Hence, we consider the following 4 bands:

$$
|s, \uparrow\rangle,|s, \downarrow\rangle,\left|p_{x}+i p_{y}, \uparrow\right\rangle,\left|p_{x}-i p_{y}, \downarrow .\right\rangle
$$

Since bulk HgTe crystalizes in the cubic zinc-blende structure, we consider 2D square lattice with two orthogonal lattice vectors $\mathbf{a}_{1}$ and $\mathbf{a}_{2}$. The Hamiltonian is written as

$$
\begin{aligned}
H_{B H Z}= & \sum_{i} \sum_{\alpha=s, p} \sum_{s_{z}= \pm} \epsilon_{\alpha} C_{i, \alpha, s_{z}}^{\dagger} C_{i, \alpha, s_{z}} \\
& -\sum_{i} \sum_{\alpha, \beta} \sum_{\mu= \pm x, \pm y} \sum_{s_{z}= \pm} t_{\mu s_{z}}^{\alpha \beta} C_{i+\mu, \alpha, s_{z}}^{\dagger} C_{i, \beta, s_{z}}
\end{aligned}
$$

where $\alpha, \beta$ are $s$ or $p$ bands, $s_{z}$ is the $z$ component of the spin, and $\mu$ specifies the four nearest-neighbor bonds. The hopping term is a matrix in the band basis:

$$
t_{\mu s_{z}}=\left(\begin{array}{cc}
t_{s s} & t_{s p} e^{i s_{z} \theta_{\mu}} \\
t_{s p} e^{-i s_{z} \theta_{\mu}} & -t_{p p}
\end{array}\right),
$$

where $\theta_{\mu}$ is the angle between the $x$ axis and the direction of the bond $\mu$, which can be $0, \frac{\pi}{2}, \pi$, and $\frac{3 \pi}{2}$.

Fourier transformation of the first term in Eq. (87) gives

$$
\sum_{i, \alpha, s_{z}} \epsilon_{\alpha} C_{i, \alpha, s_{z}}^{\dagger} C_{i, \alpha, s_{z}}=\sum_{\mathbf{k}} C_{\mathbf{k}}^{\dagger}\left(\frac{\epsilon_{s}+\epsilon_{p}}{2} I \otimes I+\frac{\epsilon_{s}-\epsilon_{p}}{2} \sigma_{z} \otimes I\right) C_{\mathbf{k}} .
$$

Similarly, the hopping term reduces in the $\mathbf{k}$ representation to

$$
\begin{gathered}
-\sum_{i, \alpha, \beta, \mu, s_{z}} t_{\mu s_{z}}^{\alpha \beta} C_{i+\mu, \alpha, s_{z}}^{\dagger} C_{i, \beta, s_{z}} \\
=-\sum_{\mathbf{k}} C_{\mathbf{k}}^{\dagger}\left[\left(t_{s s}-t_{p p}\right) \sum_{\mu}\left(\cos \mathbf{k} \cdot \mathbf{a}_{\mu}\right) I \otimes I\right.
\end{gathered}
$$

$$
\begin{gathered}
+\left(t_{s s}+t_{p p}\right) \sum_{\mu}\left(\cos \mathbf{k} \cdot \mathbf{a}_{\mu}\right) \sigma_{z} \otimes I \\
+\left(2 t_{s p} \sin \mathbf{k} \cdot \mathbf{a}_{\mathbf{1}}\right) \sigma_{y} \otimes I \\
\left.+\left(2 t_{s p} \sin \mathbf{k} \cdot \mathbf{a}_{2}\right) \sigma_{x} \otimes s_{z}\right] C_{\mathbf{k}}
\end{gathered}
$$

Therefore, by defining the following Dirac $\Gamma$ matrices

$$
\begin{gathered}
\Gamma^{1}=\sigma_{x} \otimes s_{x}=\left(\begin{array}{cc}
0 & s_{x} \\
s_{x} & 0
\end{array}\right), \\
\Gamma^{2}=\sigma_{x} \otimes s_{y}=\left(\begin{array}{cc}
0 & s_{y} \\
s_{y} & 0
\end{array}\right), \\
\Gamma^{3}=\sigma_{x} \otimes s_{z}=\left(\begin{array}{cc}
0 & s_{z} \\
s_{z} & 0
\end{array}\right), \\
\Gamma^{4}=\sigma_{y} \otimes I=\left(\begin{array}{cc}
0 & -i I \\
i I & 0
\end{array}\right), \\
\Gamma^{5}=\sigma_{z} \otimes I=\left(\begin{array}{cc}
I & 0 \\
0 & -I
\end{array}\right),
\end{gathered}
$$

the total Hamiltonian Eq. (87) is written in the form

$$
H(\mathbf{k})=d_{0}(\mathbf{k}) I+\sum_{a=1}^{5} d_{a}(\mathbf{k}) \Gamma^{a}
$$

with the coefficients

$$
\begin{aligned}
& d_{0}(\mathbf{k})=\left(\epsilon_{s}+\epsilon_{p}\right) / 2 \\
&-\left(t_{s s}-t_{s p}\right)\left(\cos \mathbf{k} \cdot \mathbf{a}_{1}+\cos \mathbf{k} \cdot \mathbf{a}_{2}\right) \\
& d_{1}(\mathbf{k})= 0 \\
& d_{2}(\mathbf{k})=0 \\
& d_{3}(\mathbf{k})=2 t_{s p} \sin \mathbf{k} \cdot \mathbf{a}_{2} \\
& d_{4}(\mathbf{k})=2 t_{s p} \sin \mathbf{k} \cdot \mathbf{a}_{1} \\
& d_{5}(\mathbf{k})=\left(\epsilon_{s}-\epsilon_{p}\right) / 2 \\
&-\left(t_{s s}+t_{s p}\right)\left(\cos \mathbf{k} \cdot \mathbf{a}_{1}+\cos \mathbf{k} \cdot \mathbf{a}_{2}\right)
\end{aligned}
$$

The energy eigenvalues are given by

$$
E(\mathbf{k})=d_{0}(\mathbf{k}) \pm \sqrt{\sum_{a} d_{a}(\mathbf{k})^{2}} .
$$

Note that, since $s$-orbital is parity even and $p$-orbital is parity odd, the inversion operator in this band basis is constructed as

$$
\Pi=\sigma_{z} \otimes I=\Gamma^{5} .
$$

One may notice that the $\Gamma$ matrices defined above have the following symmetry:

$$
\begin{aligned}
& \Theta \Gamma^{a} \Theta^{-1}= \begin{cases}-\Gamma^{a} & (a=1,2,3,4) \\
+\Gamma^{a} & (a=5)\end{cases} \\
& \Pi \Gamma^{a} \Pi^{-1}=\left\{\begin{array}{ll}
-\Gamma^{a} & (a=1,2,3,4) \\
+\Gamma^{a} & (a=5)
\end{array} .\right.
\end{aligned}
$$


Since only $\Gamma^{5}$ is even under TR and inversion, at a TRIM $\Lambda_{i}$ where the system preserves both TRS and IS, the Hamiltonian must have the form

$$
H\left(\mathbf{k}=\Lambda_{i}\right)=d_{0}\left(\Lambda_{i}\right) I+d_{5}\left(\Lambda_{i}\right) \Gamma^{5} .
$$

Also, Kramers theorem dictates that $|s, \uparrow\rangle$ and $|s, \downarrow\rangle$ are degenerate at $\Lambda_{i}$, and so are $\left|p_{x}+i p_{y}, \uparrow\right\rangle$ and $\left|p_{x}-i p_{y}, \downarrow\right\rangle$. Remembering that the $s$ - and $p$-orbital bands have even (+) and odd $(-)$ parities, respectively, we denote those degenerate states at $\Lambda_{i}$ as $|+\rangle$ and $|-\rangle$. One can easily see that the eigenvalues of $H\left(\Lambda_{i}\right)$ given by Eq. (107) for those states are

$$
\begin{aligned}
\left\langle+\left|H\left(\Lambda_{i}\right)\right|+\right\rangle & =d_{0}\left(\Lambda_{i}\right)+d_{5}\left(\Lambda_{i}\right) \equiv E_{+} \\
\left\langle-\left|H\left(\Lambda_{i}\right)\right|-\right\rangle & =d_{0}\left(\Lambda_{i}\right)-d_{5}\left(\Lambda_{i}\right) \equiv E_{-},
\end{aligned}
$$

because $\left\langle+\left|\Gamma^{5}\right|+\right\rangle=1$ and $\left\langle-\left|\Gamma^{5}\right|-\right\rangle=-1$ due to Eq. (104).

When $d_{5}\left(\Lambda_{i}\right)>0$ at a TRIM $\Lambda_{i}, E_{+}>E_{-}$and the $|-\rangle$state is occupied at that $\Lambda_{i}$ if the system is half-filled; in this case, the parity of the occupied state at $\Lambda_{i}, \delta\left(\Lambda_{i}\right)$ in Eq. (85), is -1 . On the other hand, when $d_{5}\left(\Lambda_{i}\right)<0, E_{+}$becomes smaller than $E_{\text {- }}$ and hence the $|+\rangle$ state is occupied, making $\delta\left(\Lambda_{i}\right)=+1$. Therefore, in the present 4-band model one may conclude

$$
\delta\left(\Lambda_{i}\right)=-\operatorname{sgn}\left[d_{5}\left(\Lambda_{i}\right)\right] .
$$

If we set $\left|\mathbf{a}_{1}\right|=\left|\mathbf{a}_{2}\right|=\pi$, the four TRIMs are $(0,0),(0,1)$, $(1,0)$, and (1, 1). Putting Eq. (102) into Eq. (109), one obtains

$$
\delta\left(\Lambda\left(n_{1}, n_{2}\right)\right)=-\operatorname{sgn}\left[\frac{\epsilon_{s}-\epsilon_{p}}{2}-\left(t_{s s}+t_{s p}\right)\left\{(-1)^{n_{1}}+(-1)^{n_{2}}\right\}\right] .
$$

This leads to the following conclusion regarding the topology of this 4-band system:

- If $\epsilon_{s}-\epsilon_{p}>4\left(t_{s s}+t_{s p}\right), \delta<0$ for all $\Lambda_{i}$, which leads to the $Z_{2}$ invariant $v$ given by Eq. (86) to become 0 . This means that the system is a trivial insulator.

- If $\epsilon_{s}-\epsilon_{p}<4\left(t_{s s}+t_{s p}\right), \delta>0$ at $\mathbf{k}=0$ but $\delta<0$ for other three TRIMs, which leads to $v=1$. This means that the system is a TI.

It is instructive to note that in the trivial state the band order is $E_{-}<E_{+}$, which means $p$-orbital bands lie below the $s$-orbital bands, which is normal for a band insulator. When the system becomes a TI, the band order flips only at the $\Gamma$ point in the $2 \mathrm{D} \mathrm{BZ}$, and such a band inversion occurring at an odd number of TRIMs in the BZ is the key to realizing the nontrivial $Z_{2}$ topology. In the case of $\mathrm{HgTe}$, this band inversion occurs due to strong SOC. Therefore, this example tells us that a viable strategy for discovering a TI is to look for materials having strong SOC that causes band inversion at an odd number of TRIMs in the BZ.

\section{TI Materials Discovered to Date}

Table I gives a summary of the TI materials discussed in this section. This table lists only those materials that have been experimentally addressed as of May 2013, since there are too many materials that have been predicted to be TIs but not tested experimentally.

\subsection{Two-dimensional TIs}

The first material that was experimentally identified as a TR-invariant $\mathrm{TI}$ was $\mathrm{CdTe} / \mathrm{HgTe} / \mathrm{CdTe}$ quantum well, ${ }^{31}$ ) namely, a thin layer of $\mathrm{HgTe}$ sandwiched by CdTe. This is a 2D system where the degree of freedom for the perpendicular direction is quenched due to the quantum confinement of the electronic states in the $\mathrm{HgTe}$ unit and the resulting subband formation. Both $\mathrm{HgTe}$ and CdTe crystallize in zinc-blende structure, and their superlattices have been actively studied because of their application to infrared detectors. Therefore, the necessary technology to synthesize the required quantum well was already developed before the prediction of its TI nature, although it involves a very specialized molecular-beam epitaxy (MBE) technique to deal with mercury. ${ }^{31)}$

As we have seen in the analysis of the BHZ model in the previous section, the inversion between $p$ - and $s$-orbital bands is essential for the system to obtain the TI nature. Bulk HgTe realizes such a band inversion, while CdTe does not. Therefore, $\mathrm{HgTe}$ is a good starting material for conceiving a TI phase; however, there is a problem in the band structure of bulk $\mathrm{HgTe}$, that is, a crystal-symmetry-protected degeneracy at the $\Gamma$ point makes the system to be intrinsically a zero-gap semiconductor, ${ }^{30)}$ which means that there is no band gap between the $p$ - and $s$-orbital bands and the system is not qualified as an insulator. However, by sandwiching $\mathrm{HgTe}$ by CdTe, which has slightly larger lattice constant, the epitaxial strain exerted on HgTe breaks the cubic lattice symmetry and leads to a gap opening, and hence the system can become a genuine insulator. It was predicted by $\mathrm{BHZ}$ that above a certain critical thickness the strained HgTe unit retains the band inversion and should be a TI. ${ }^{30}$ ) This prediction was confirmed by a group at the University of Würzburg led by Molenkamp via transport experiment of micro-fabricated samples. ${ }^{31)}$ They found that, when the thickness of the HgTe unit is above the critical thickness $d_{c}$ of $6.3 \mathrm{~nm}$, their samples show a "negative energy gap" (i.e. band is inverted) and quantization of the conductance to $2 e^{2} / h$ in zero magnetic field was observed when the chemical potential is tuned into the gap (Fig. 2). In contrast, when the thickness is below $d_{c}$, the band inversion is lost and they observed diverging resistivity.

The Würzburg group later reported the observation of nonlocal transport, ${ }^{69)}$ which gave further support to the existence of an edge state. They also showed that the 1D edge state responsible for the quantized transport in the 2D TI phase is likely to be helically spin polarized, by fabricating an elaborate device structure which relies on the conventional spin Hall effect that occurs in metallic (doped) $\mathrm{HgTe}^{70)}$

The $\mathrm{CdTe} / \mathrm{HgTe} / \mathrm{CdTe}$ quantum well can be made very clean with the carrier mobility of up to $\sim 10^{5} \mathrm{~cm}^{2} / \mathrm{Vs}$, which makes it possible to study quantum transport properties. On the other hand, the drawback of this system is that the bulk band gap that opens due to the epitaxial strain is very small (up to $\sim 10 \mathrm{meV}$, depending on the thickness ${ }^{71)}$ ), which makes the detection of the TI phase to be possible only at very low 
Table I. Summary of topological insulator materials that have bee experimentally addressed. The definition of (1;111) etc. is introduced in Sec. 3.7. (In this table, S.S., P.T., and SM stand for surface state, phase transition, and semimetal, respectively.)

\begin{tabular}{|c|c|c|c|c|c|}
\hline Type & Material & Band gap & Bulk transport & Remark & Reference \\
\hline $2 \mathrm{D}, v=1$ & $\mathrm{CdTe} / \mathrm{HgTe} / \mathrm{CdTe}$ & $<10 \mathrm{meV}$ & insulating & high mobility & 31) \\
\hline $2 \mathrm{D}, v=1$ & $\mathrm{AlSb} / \mathrm{InAs} / \mathrm{GaSb} / \mathrm{AlSb}$ & $\sim 4 \mathrm{meV}$ & weakly insulating & gap is too small & 73) \\
\hline 3D $(1 ; 111)$ & $\mathrm{Bi}_{1-x} \mathrm{Sb}_{x}$ & $<30 \mathrm{meV}$ & weakly insulating & complex S.S. & $36,40)$ \\
\hline 3D $(1 ; 111)$ & $\mathrm{Sb}$ & semimetal & metallic & complex S.S. & 39) \\
\hline $3 \mathrm{D}(1 ; 000)$ & $\mathrm{Bi}_{2} \mathrm{Se}_{3}$ & $0.3 \mathrm{eV}$ & metallic & simple S.S. & 94) \\
\hline $3 \mathrm{D}(1 ; 000)$ & $\mathrm{Bi}_{2} \mathrm{Te}_{3}$ & $0.17 \mathrm{eV}$ & metallic & distorted S.S. & $95,96)$ \\
\hline 3D $(1 ; 000)$ & $\mathrm{Sb}_{2} \mathrm{Te}_{3}$ & $0.3 \mathrm{eV}$ & metallic & heavily $p$-type & 97) \\
\hline $3 \mathrm{D}(1 ; 000)$ & $\mathrm{Bi}_{2} \mathrm{Te}_{2} \mathrm{Se}$ & $\sim 0.2 \mathrm{eV}$ & reasonably insulating & $\rho_{x x}$ up to $6 \Omega \mathrm{cm}$ & $102,103,105)$ \\
\hline $3 \mathrm{D}(1 ; 000)$ & $(\mathrm{Bi}, \mathrm{Sb})_{2} \mathrm{Te}_{3}$ & $<0.2 \mathrm{eV}$ & moderately insulating & mostly thin films & 193) \\
\hline $3 \mathrm{D}(1 ; 000)$ & $\mathrm{Bi}_{2-x} \mathrm{Sb}_{x} \mathrm{Te}_{3-y} \mathrm{Se}_{y}$ & $<0.3 \mathrm{eV}$ & reasonably insulating & Dirac-cone engineering & $107,108,212)$ \\
\hline $3 \mathrm{D}(1 ; 000)$ & $\mathrm{Bi}_{2} \mathrm{Te}_{1.6} \mathrm{~S}_{1.4}$ & $0.2 \mathrm{eV}$ & metallic & $n$-type & 210) \\
\hline $3 \mathrm{D}(1 ; 000)$ & $\mathrm{Bi}_{1.1} \mathrm{Sb}_{0.9} \mathrm{Te}_{2} \mathrm{~S}$ & $0.2 \mathrm{eV}$ & moderately insulating & $\rho_{x x}$ up to $0.1 \Omega \mathrm{cm}$ & 210) \\
\hline $3 \mathrm{D}(1 ; 000)$ & $\mathrm{Sb}_{2} \mathrm{Te}_{2} \mathrm{Se}$ & $?$ & metallic & heavily $p$-type & 102) \\
\hline $3 \mathrm{D}(1 ; 000)$ & $\mathrm{Bi}_{2}(\mathrm{Te}, \mathrm{Se})_{2}(\mathrm{Se}, \mathrm{S})$ & $0.3 \mathrm{eV}$ & semi-metallic & natural Kawazulite & 211) \\
\hline $3 \mathrm{D}(1 ; 000)$ & $\mathrm{TlBiSe}_{2}$ & $\sim 0.35 \mathrm{eV}$ & metallic & simple S.S., large gap & $110-112)$ \\
\hline $3 \mathrm{D}(1 ; 000)$ & $\mathrm{TlBiTe}_{2}$ & $\sim 0.2 \mathrm{eV}$ & metallic & distorted S.S. & 112) \\
\hline 3D $(1 ; 000)$ & $\mathrm{TlBi}(\mathrm{S}, \mathrm{Se})_{2}$ & $<0.35 \mathrm{eV}$ & metallic & topological P.T. & $116,117)$ \\
\hline $3 \mathrm{D}(1 ; 000)$ & $\mathrm{PbBi}_{2} \mathrm{Te}_{4}$ & $\sim 0.2 \mathrm{eV}$ & metallic & S.S. nearly parabolic & $121,124)$ \\
\hline $3 \mathrm{D}(1 ; 000)$ & $\mathrm{PbSb}_{2} \mathrm{Te}_{4}$ & $?$ & metallic & $p$-type & 121) \\
\hline $3 \mathrm{D}(1 ; 000)$ & $\mathrm{GeBi}_{2} \mathrm{Te}_{4}$ & $0.18 \mathrm{eV}$ & metallic & $n$-type & $102,119,120)$ \\
\hline $3 \mathrm{D}(1 ; 000)$ & $\mathrm{PbBi}_{4} \mathrm{Te}_{7}$ & $0.2 \mathrm{eV}$ & metallic & heavily $n$-type & 125) \\
\hline $3 \mathrm{D}(1 ; 000)$ & $\mathrm{GeBi}_{4-x} \mathrm{Sb}_{x} \mathrm{Te}_{7}$ & $0.1-0.2 \mathrm{eV}$ & metallic & $n(p)$ type at $x=0(1)$ & 126) \\
\hline $3 \mathrm{D}(1 ; 000)$ & $(\mathrm{PbSe})_{5}\left(\mathrm{Bi}_{2} \mathrm{Se}_{3}\right)_{6}$ & $0.5 \mathrm{eV}$ & metallic & natural heterostructure & 130) \\
\hline $3 \mathrm{D}(1 ; 000)$ & $\left(\mathrm{Bi}_{2}\right)\left(\mathrm{Bi}_{2} \mathrm{Se}_{2.6} \mathrm{~S}_{0.4}\right)$ & semimetal & metallic & $\left(\mathrm{Bi}_{2}\right)_{n}\left(\mathrm{Bi}_{2} \mathrm{Se}_{3}\right)_{m}$ series & 127) \\
\hline $3 \mathrm{D}(1 ; 000)$ & $\left(\mathrm{Bi}_{2}\right)\left(\mathrm{Bi}_{2} \mathrm{Te}_{3}\right)_{2}$ & $?$ & $?$ & no data published yet & 128) \\
\hline 3D TCI & SnTe & $0.3 \mathrm{eV}(4.2 \mathrm{~K})$ & metallic & Mirror TCI, $n_{\mathcal{M}}=-2$ & 62) \\
\hline 3D TCI & $\mathrm{Pb}_{1-x} \mathrm{Sn}_{x} \mathrm{Te}$ & $<0.3 \mathrm{eV}$ & metallic & Mirror TCI, $n_{\mathcal{M}}=-2$ & 164) \\
\hline 3D TCI & $\mathrm{Pb}_{0.77} \mathrm{Sn}_{0.23} \mathrm{Se}$ & invert with $T$ & metallic & Mirror TCI, $n_{\mathcal{M}}=-2$ & 162) \\
\hline $2 \mathrm{D}, v=1 ?$ & Bi bilayer & $\sim 0.1 \mathrm{eV}$ & $?$ & not stable by itself & $82,83)$ \\
\hline 3D $(1 ; 000) ?$ & $\mathrm{Ag}_{2} \mathrm{Te}$ & $?$ & metallic & famous for linear MR & $134,135)$ \\
\hline 3D $(1 ; 111) ?$ & $\mathrm{SmB}_{6}$ & $20 \mathrm{meV}$ & insulating & possible Kondo TI & $140-143)$ \\
\hline $3 \mathrm{D}(0 ; 001) ?$ & $\mathrm{Bi}_{14} \mathrm{Rh}_{3} \mathrm{I}_{9}$ & $0.27 \mathrm{eV}$ & metallic & possible weak 3D TI & 145) \\
\hline 3D $(1 ; 000) ?$ & $R \operatorname{BiPt}(R=\mathrm{Lu}, \mathrm{Dy}, \mathrm{Gd})$ & zero gap & metallic & evidence negative & 152) \\
\hline Weyl SM? & $\mathrm{Nd}_{2}\left(\mathrm{Ir}_{1-x} \mathrm{Rh}_{x}\right)_{2} \mathrm{O}_{7}$ & zero gap & metallic & too preliminary & 158) \\
\hline
\end{tabular}

temperature. Also, since the synthesis of $\mathrm{CdTe} / \mathrm{HgTe} / \mathrm{CdTe}$ quantum wells requires dedicated MBE machines, the sources of samples for basic physics experiments are currently very limited, which has made the progress of experimental studies of 2D TIs (QSH insulators) relatively slow.

Recently, another 2D TI system, AlSb/InAs/GaSb/AlSb quantum well, was theoretically predicted ${ }^{72)}$ and experimentally confirmed. ${ }^{73-75)}$ The essential workings of this system are the following: The valence-band top of GaSb lies above the conduction-band bottom of InAs. Hence, when InAs and $\mathrm{GaSb}$ are in direct contact and they are both quantum confined (by the outer units of AlSb which has a large band gap and works as a barrier), the resulting hole subband in GaSb may lie above the electron subband in InAs, and therefore the band order of this quantum well is inverted. The band gap in this quantum well arises from anti-crossing of the two subbands at finite momentum and hence is very small $(\sim 4 \mathrm{meV})$, which makes clean observation of the helical edge state very difficult. ${ }^{73,76)}$ Reasonably convincing evidence for the TI phase was obtained via observation of the $2 e^{2} / h$ quantization of the zero-bias Andreev reflection conductance through $\mathrm{Nb}$ point contacts, ${ }^{74)}$ but more recently, direct observation of the conductance quantization to $2 e^{2} / h$ has been achieved by introducing disorder to the InAs/ $\mathrm{GaSb}$ interface by $\mathrm{Si}$ doping to localize the unwanted bulk carriers. ${ }^{75)}$

Other possible candidates of $2 \mathrm{D}$ TIs include $\mathrm{Bi}$ bilayer, ${ }^{77)} \mathrm{Na}_{2} \mathrm{IrO}_{3}{ }^{78)}$ and graphene with artificially enhanced SOC. $^{79,80)}$ In particular, for the Bi bilayer there have been experimental efforts to address its topological nature, ${ }^{81-83)}$ and suggestive evidence for the existence of edge states has been 
reported. ${ }^{82,83)}$ It is worth mentioning that silicene, an ana$\log$ of graphene consisting of silicon atoms instead of carbon atoms, ${ }^{84)}$ has also been predicted to be a 2D TI with a high tunability. ${ }^{85)}$ Since the experimental studies of 2D TIs have been hindered by the scarcity of samples, new discoveries of 2D TI materials are strongly call for.

\subsection{Three-dimensional TIs}

As already mentioned in Sec. 2, the first 3D TI material that was experimentally identified was $\mathrm{Bi}_{1-x} \mathrm{Sb}_{x},{ }^{36)}$ following the very specific prediction by $\mathrm{Fu}$ and Kane. ${ }^{35)}$ This material is an alloy of $\mathrm{Bi}$ and $\mathrm{Sb}$ and it naturally possesses the two essential features, (i) band inversion at an odd number of TRIMs and (ii) opening of a bulk band gap, in the Sb concentration range of 0.09 to 0.23 . $^{86)}$ The $3 \mathrm{D} Z_{2}$ invariant has been identified as $(1 ; 111)$.

Unfortunately, it turned out that this system is not very suitable for detailed studies of the topological surface state due to the complicated surface-state structure, ${ }^{36,40)}$ as can be seen in Fig. 3. This is because its parent material, Bi, already harbors prominent spin-non-degenerate surface states due to the strong Rashba effect on the surface of this material. ${ }^{87)}$ In $\mathrm{Bi}_{1-x} \mathrm{Sb}_{x}$, such non-topological, Rashba-split surface states are responsible for 2 or 4 Fermi-level crossings of the surface states (depending on the chemical potential), ${ }^{36,40)}$ and the topological one contributes just one additional Fermi-level crossing. First-principle calculations of the surface states of $\mathrm{Bi}_{1-x} \mathrm{Sb}_{x}$ have been reported, ${ }^{88,89)}$ but the predicted surfacestate structure does not really agree with the experimental results. Such an incomplete understanding of the nature of the surface state is partly responsible for confusions occasionally seen in interpretations of experimental data. For example, in the STS work which addressed the protection from backscattering in the surface state of $\mathrm{Bi}_{1-x} \mathrm{Sb}_{x},{ }^{38)}$ the analysis considered the spin polarizations of only those surface states that are also present in topologically trivial $\mathrm{Bi}^{87}$ ) and yet, it was concluded that the result gives evidence for topological protection.

Nevertheless, $\mathrm{Bi}_{1-x} \mathrm{Sb}_{x}$ is unique among known 3D TI materials in that it has an intrinsically high 2D carrier mobility of $\sim 10^{4} \mathrm{~cm}^{2} / \mathrm{Vs}$ (despite the fact that it is an alloy), which makes it easy to study novel 2D quantum transport. ${ }^{37,90)}$ Also, it is relatively easy for this system to reduce the bulk carrier density to $\sim 10^{16} \mathrm{~cm}^{-3}$ in high-quality single crystals, making it possible, for example, to perform Landau level spectroscopy of the surface states via magneto-optics. ${ }^{91)}$ The bulk band gap of $\mathrm{Bi}_{1-x} \mathrm{Sb}_{x}$ is not very large (up to $\sim 30 \mathrm{meV}$ depending on $x),{ }^{86)}$ but it is large enough to detect the $2 \mathrm{D}$ transport properties at $4 \mathrm{~K}$.

Along with $\mathrm{Bi}_{1-x} \mathrm{Sb}_{x}$, Fu and Kane predicted ${ }^{35)}$ that $\mathrm{HgTe}$, $\alpha$-phase of $\mathrm{Sn}$ (called "gray tin"), and $\mathrm{Pb}_{1-x} \mathrm{Sn}_{x} \mathrm{Te}$ would become 3D TIs under uniaxial strain to break the cubic lattice symmetry. They also suggested that $\mathrm{Bi}_{2} \mathrm{Te}_{3}$ would be a candidate, but they did not perform band calculations to elucidate

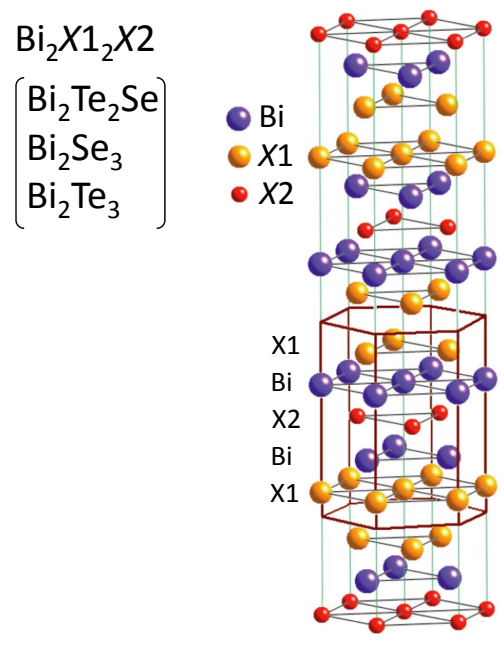

Fig. 7. (Color online) Crystal structure of tetradymite chalcogenides, $\mathrm{Bi}_{2} \mathrm{Se}_{3}(X 1=X 2=\mathrm{Se}), \mathrm{Bi}_{2} \mathrm{Te}_{3}(X 1=X 2=\mathrm{Te})$, and $\mathrm{Bi}_{2} \mathrm{Te}_{2} \mathrm{Se}(X 1=\mathrm{Te}$; $X 2=\mathrm{Se})$. The quintuple layer $(\mathrm{QL})$ enclosed by brown cage is the building block of this type of materials. The stack of the QLs are in the -A-B-CA-B-C- manner, and hence the unit cell consists of a stack of 3 QLs. The $\mathrm{Bi}_{2-x} \mathrm{Sb}_{x} \mathrm{Te}_{3-y} \mathrm{Se}_{y}(y \geq 1)$ compound also takes this structure, in which $X 2=$ $\mathrm{Se}, X 1=\mathrm{Se} / \mathrm{Te}$, and the original Bi site becomes a mixture of Bi/Sb. Taken from Ref. 103; copyright American Physical Society (2010).

the parity eigenvalues. Such calculations were done by Zhang et al., ${ }^{92)}$ who came up with a concrete prediction that $\mathrm{Bi}_{2} \mathrm{Se}_{3}$, $\mathrm{Bi}_{2} \mathrm{Te}_{3}$, and $\mathrm{Sb}_{2} \mathrm{Te}_{3}$ should be $3 \mathrm{D}$ TIs but $\mathrm{Sb}_{2} \mathrm{Se}_{3}$ is not; furthermore, Zhang et al. proposed a low-energy effective model to describe the bulk band structure of this class of materials. This model, with some corrections made later, ${ }^{93)}$ has become a popular model for theoretically discussing the properties of a 3D TI. Experimentally, existence of a single Dirac-cone surface state was reported in 2009 for $\mathrm{Bi}_{2} \mathrm{Se}_{3}$ by Xia et al. ${ }^{94)}$ and for $\mathrm{Bi}_{2} \mathrm{Te}_{3}$ by Chen et al. ${ }^{95)}$ and also by Hsieh et al.; ${ }^{96)} \mathrm{Sb}_{2} \mathrm{Te}_{3}$ was measured by Hsieh et al. ${ }^{96)}$ along with $\mathrm{Bi}_{2} \mathrm{Te}_{3}$, but the existence of the topological surface state was left unconfirmed due to the heavily $p$-type nature of the measured samples. The topological nature of $\mathrm{Sb}_{2} \mathrm{Te}_{3}$ was confirmed only recently in thin-film samples using STS. ${ }^{97)}$

$\mathrm{Bi}_{2} \mathrm{Se}_{3}, \mathrm{Bi}_{2} \mathrm{Te}_{3}$, and $\mathrm{Sb}_{2} \mathrm{Te}_{3}$ all crystallize in tetradymite structure, which consists of covalently bonded quintuple layers (e.g. Se-Bi-Se-Bi-Se) that are stacked in -A-B-C-A-B-Cmanner and are weakly interacting with van der Waals force (Fig. 7); therefore, those materials cleave easily between quintuple layers (QLs). Since each QL is about $1 \mathrm{~nm}$ thick, the lattice constant along the $c$-axis is about $3 \mathrm{~nm}$.

The $3 \mathrm{D} Z_{2}$ invariant of these tetradymite systems is $(1 ; 000)$, which means that topological Dirac-cone surface state is centered at the $\bar{\Gamma}$ point of the surface BZ (Fig. 8). This simplicity of the topological surface state and the absence of nontopological surface states make those materials well suited for experimentally addressing the properties of the topological surface state. Also, single-crystal growth of those materials is 
(a)
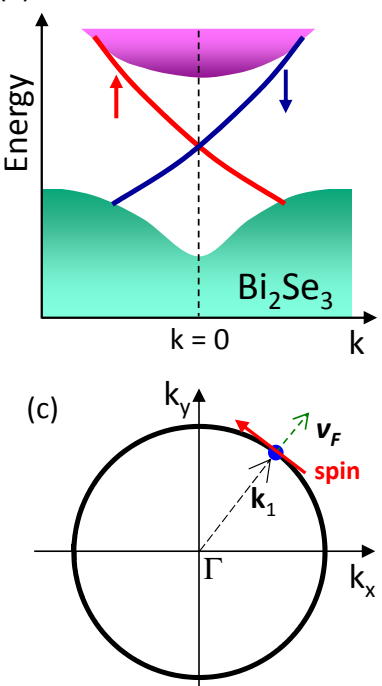

(b)

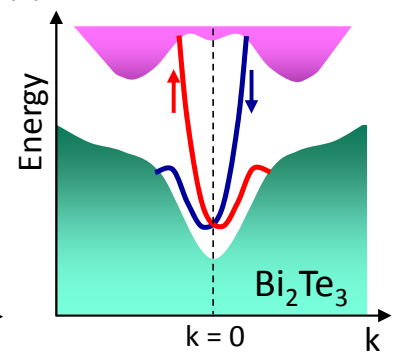

(d)

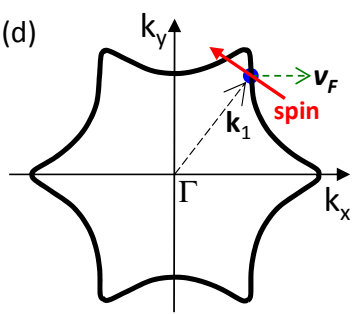

Fig. 8. (Color online) Schematic bulk and surface band structures of (a) $\mathrm{Bi}_{2} \mathrm{Se}_{3}$ and (b) $\mathrm{Bi}_{2} \mathrm{Te}_{3}$. Note that the surface states are spin non-degenerate and are helically spin polarized. Representative constant-energy contours of the Dirac cones for (c) $\mathrm{Bi}_{2} \mathrm{Se}_{3}$ and (d) $\mathrm{Bi}_{2} \mathrm{Te}_{3}$ are also schematically shown. Note that the spin vector is always perpendicular to the wave vector $\mathbf{k}_{1}$ in both $\mathrm{Bi}_{2} \mathrm{Se}_{3}$ and $\mathrm{Bi}_{2} \mathrm{Te}_{3}$, but the Fermi velocity vector $\mathbf{v}_{F}$ can be non-orthogonal to the spin vector in $\mathrm{Bi}_{2} \mathrm{Te}_{3}$ due to the hexagonal warping, which leads to strong quasiparticle interference.

relatively simple, which made it easy for many experimentalists to start working on them. Furthermore, the bulk band gap of $\mathrm{Bi}_{2} \mathrm{Se}_{3}$ is relatively large, $0.3 \mathrm{eV}$, and thus one can see technological relevance that topological properties of this material may potentially be exploited at room temperature. All those factors helped initiate a surge of research activities on 3D TIs.

The surface-state structure of $\mathrm{Bi}_{2} \mathrm{Se}_{3}$ is relatively simple and presents an almost idealized Dirac cone with only slight curvature, as shown schematically in Fig. 8(a). In contrast, the surface state of $\mathrm{Bi}_{2} \mathrm{Te}_{3}$ is a bit more complicated [see Fig. 8(b)] and the Dirac point is located beneath the top of the valence band, which makes it difficult to probe the surface transport properties near the Dirac point without being disturbed by bulk carriers in $\mathrm{Bi}_{2} \mathrm{Te}_{3}$.

Another difference between the two materials is that the constant-energy contour of the Dirac cone is almost spherical in $\mathrm{Bi}_{2} \mathrm{Se}_{3}$ [Fig. 8(c)], while it presents significant hexagonal warping in $\mathrm{Bi}_{2} \mathrm{Te}_{3}$ [Fig. 8(d)]. This warping is caused by a $k^{3}$ term which stems from cubic Dresselhaus spin-orbit coupling at the surface of rhombohedral crystal systems. ${ }^{98)}$ Intriguingly, this hexagonal warping gives rise to peculiar physics such as strong quasiparticle interference ${ }^{99,100)}$ and appearance of a finite out-of-plane spin polarization. ${ }^{101)}$

While $\mathrm{Bi}_{2} \mathrm{Se}_{3}$ and $\mathrm{Bi}_{2} \mathrm{Te}_{3}$ are conceptually simple TI materials, their chemistry is not so simple and they are always degenerately doped due to naturally occurring crystalline defects, which cause their transport properties to be dominated

by bulk carriers. In this respect, a promising TI material having the tetradymite structure is $\mathrm{Bi}_{2} \mathrm{Te}_{2} \mathrm{Se}$, which has a chalcogen-ordered structure shown in Fig. 7. The topological surface state of this material was first reported by $\mathrm{Xu}$ et $a l$. in a preprint that has not been published, ${ }^{102)}$ and they reported metallic $n$-type nature for the stoichiometric composition of this material. However, Ren et al. discovered ${ }^{103)}$ that by growing crystals from a slightly Se-rich starting composition of $\mathrm{Bi}_{2} \mathrm{Te}_{1.95} \mathrm{Se}_{1.05}$, one can obtain crystals showing a large bulk resistivity exceeding $1 \Omega \mathrm{cm}$. Furthermore, they demonstrated that in such crystals, the chemical potential is located within the bulk band gap and one can observe clear $\mathrm{SdH}$ oscillations coming from the topological surface state, which contributed $\sim 6 \%$ of the total conductance in a $260-\mu \mathrm{m}$-thick bulk crystal. ${ }^{103)}$ This value is to be contrasted with the preceding surface transport study by Qu et al. on $\mathrm{Bi}_{2} \mathrm{Te}_{3}$, which found the surface contribution of only $\sim 0.3 \%$ in a $100-\mu \mathrm{m}$ thick sample. ${ }^{104)} \mathrm{In}$ fact, $\mathrm{Bi}_{2} \mathrm{Te}_{2} \mathrm{Se}$ was the first $3 \mathrm{D}$ TI material to present a reasonably bulk-insulating behavior, which opened the door for detailed transport studies of the topological surface state. This discovery by Ren $e t$ al. was followed by an independent report by Xiong et al., ${ }^{105)}$ who reported an even larger resistivity of $6 \Omega \mathrm{cm}$. Detailed defect chemistry in $\mathrm{Bi}_{2} \mathrm{Te}_{2} \mathrm{Se}$ was discussed by Jia et al. ${ }^{106)}$ In passing, a tetradymite compound having a similar chalcogen-ordered structure, $\mathrm{Sb}_{2} \mathrm{Te}_{2} \mathrm{Se}$, has also been confirmed to be topologi$\mathrm{cal},{ }^{102)}$ but it is heavily $p$-type doped.

Ren et al. tried to further improve the bulk-insulating property of $\mathrm{Bi}_{2} \mathrm{Te}_{2} \mathrm{Se}$, and they reported two possible routes. One is to expand the phase space of the compositions into $\mathrm{Bi}_{2-x} \mathrm{Sb}_{x} \mathrm{Te}_{3-y} \mathrm{Se}_{y},{ }^{107,108)}$ and they found a series of particular combinations of $(x, y)$ where the samples show maximally bulk-insulating behavior. ${ }^{107)}$ (This material is discussed in detail in Sec. 7.) The second route to improve the insulating property of $\mathrm{Bi}_{2} \mathrm{Te}_{2} \mathrm{Se}$ is to employ $\mathrm{Sn}$ doping to the $\mathrm{Bi}$ site. ${ }^{109)}$ Using both routes, it has been shown to be possible to achieve surface-dominated transport in bulk single crystals. ${ }^{108,109)}$

The discovery of tetradymite TI materials demonstrated that elucidating the parity eigenvalues based on $a b$ initio band calculations is a practical and powerful strategy for making predictions for new TI materials. In fact, the next TI material discovered after $\mathrm{Bi}_{2} \mathrm{Se}_{3}$ and $\mathrm{Bi}_{2} \mathrm{Te}_{3}$ was $\mathrm{TlBiSe}_{2}$, and its discovery, ${ }^{110-112)}$ done in 2010 , was also led by theoretical predictions. ${ }^{113,114)}$ By 2010, the competition in the TI research was already very heated and two theoretical groups independently made predictions that thallium-based III-V-VI 2 ternary chalcogenides $\mathrm{Tl}^{\prime} X_{2}\left[M^{\prime}=\mathrm{Bi}\right.$ and $\mathrm{Sb} ; X=\mathrm{S}$, Se, and Te; see Fig. 9(a)] should be TIs; experimentally, the TI nature of $\mathrm{TlBiSe}_{2}$ was reported first by Sato et al., ${ }^{110)}$ which was soon followed by independent reports by Kuroda et al. ${ }^{111)}$ and by Chen et al. ${ }^{112)}$ The latter also confirmed $\mathrm{TlBiTe}_{2}$ to be a TI. ${ }^{112)}$ The surface state structure of $\mathrm{TlBiSe}_{2}$ is similar to that in $\mathrm{Bi}_{2} \mathrm{Se}_{3}$ [see Fig. 9(b)], and its simplicity makes it suitable for studying fundamental properties of the Dirac cone with- 


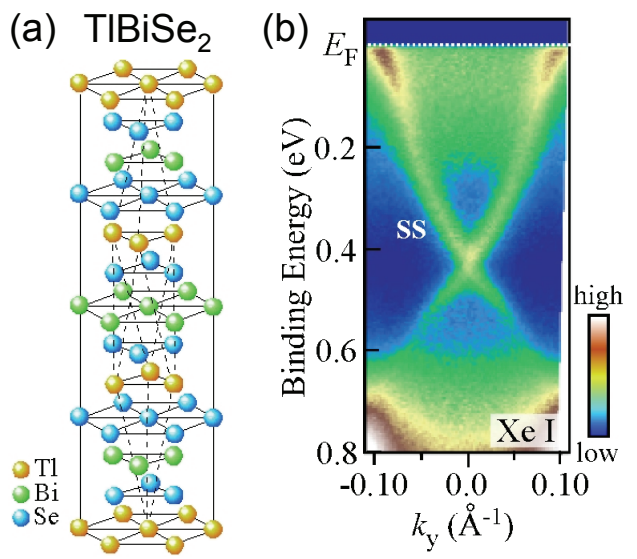

Fig. 9. (Color online) (a) Crystal structure of TlBiSe 2 . (b) Topological surface state observed in $\mathrm{TlBiSe}_{2}$ by ARPES experiment using Xe I line ( $h v$ $=8.437 \mathrm{eV}$ ). Taken from Ref. 110; copyright American Physical Society (2010).

out being bothered by additional complications. Also, Sato et al. elucidated that the bulk band gap of $\mathrm{TlBiSe}_{2}$ is $0.35 \mathrm{eV},{ }^{110}$ ) which is among the largest so far reported for 3D TI materials.

Intriguingly, $\mathrm{TlBiS}_{2}$, which was initially predicted to be a $\mathrm{TI},{ }^{113,114)}$ turned out to be non-topological. This indicates that $a b$ initio calculations are not always accurate in every details, and one should interpret the results with due care. ${ }^{115)}$ The non-topological nature of $\mathrm{TlBiS}_{2}$ naturally points to a topological phase transition, accompanied by a closing of the bulk band gap, occurring in the solid solution $\mathrm{TlBi}\left(\mathrm{S}_{1-x} \mathrm{Se}_{x}\right)_{2}$. Such a transition was studied independently by $\mathrm{Xu}$ et $a{ }^{116)}$ and by Sato et al. ${ }^{117)}$ and was found to be located at $x=0.5$. Intriguingly, Sato et al. found an unexpected gap opening at the Dirac point when the composition is on the topological side and is close to the transition. ${ }^{117)}$ This is surprising, because such a gap opening points to a lifting of the Kramers degeneracy, but in this system TRS is not explicitly broken. While the origin of this "Dirac gap" is not clear and its existence has been a matter of debate, a recent follow-up experiment substantiated its unique properties. ${ }^{118)}$

After the discovery of $\mathrm{TlBiSe}_{2}$, various ternary compounds have been identified to be $3 \mathrm{D}$ TIs. Among them, $\mathrm{GeBi}_{2} \mathrm{Te}_{4}$ was suggested to be an intrinsic insulator. ${ }^{102,119)}$ However, a follow-up work by Okamoto et al. showed that $\mathrm{GeBi}_{2} \mathrm{Te}_{4}$ is naturally electron doped. ${ }^{120}$ ) $\mathrm{The}_{\mathrm{GeBi}} \mathrm{Te}_{4}$ crystals grown in the present author's laboratory are also highly conducting with $n$-type carriers.

Another relatively new TI compound is $\mathrm{Pb}\left(\mathrm{Bi}_{1-x} \mathrm{Sb}_{x}\right)_{2} \mathrm{Te}_{4}$, for which the observation of a topological surface state, as well as a switching between $p$ - and $n$-type surface carriers with $x$, has been reported by Souma et al. ${ }^{121)}$ This work was also motivated by theoretical predictions. ${ }^{102,122,123)}$ Kuroda et al. independently reported the topological surface state for one of its end members, $\mathrm{PbBi}_{2} \mathrm{Te}_{4} \cdot{ }^{124)}$ Interestingly, $\mathrm{Pb}$-based materials form various homologous series of compounds, such as $n \mathrm{PbTe}-m \mathrm{Bi}_{2} \mathrm{Te}_{3}$. In fact, $\mathrm{PbBi}_{2} \mathrm{Te}_{4}$ can be viewed as a member of this homologous series with $n=m=1$. Observation of a topological surface state was also reported for its $n=1, m=2$ member, $\mathrm{PbBi}_{4} \mathrm{Te}_{7}{ }^{125)}$ In passing, Ge-based materials form similar homologous series as $\mathrm{Pb}$-based ones. $\mathrm{GeBi}_{2} \mathrm{Te}_{4}{ }^{102,119)}$ and $\mathrm{GeBi}_{4-x} \mathrm{Sb}_{x} \mathrm{Te}_{7}{ }^{126)}$ are examples with $n$ $=m=1$ and $n=1, m=2$, respectively.

It is known that $\mathrm{Bi}$ bilayer and $\mathrm{Bi}_{2} \mathrm{Se}_{3}$ (or $\mathrm{Bi}_{2} \mathrm{Te}_{3}$ ) also form a homologous series of compounds, $\left(\mathrm{Bi}_{2}\right)_{n}\left(\mathrm{Bi}_{2} X_{3}\right)_{m}(X$ $=\mathrm{Se}, \mathrm{Te}) .{ }^{127)}$ Topological surface state has been reported for a partially-S-substituted variant, $\left(\mathrm{Bi}_{2}\right)\left(\mathrm{Bi}_{2} \mathrm{Se}_{2.6} \mathrm{~S}_{0.4}\right)$ (= $\left.\mathrm{Bi}_{4} \mathrm{Se}_{2.6} \mathrm{~S}_{0.4}\right)$ which is a semimetal. ${ }^{127)}$ In this type of homologous series, $\left(\mathrm{Bi}_{2}\right)\left(\mathrm{Bi}_{2} \mathrm{Te}_{3}\right)_{2}(=\mathrm{BiTe})$ has also been synthesized and was found to be topological ${ }^{128)}$

As mentioned in conjunction with $\mathrm{Bi}_{1-x} \mathrm{Sb}_{x}$, one of the initial predictions of $\mathrm{Fu}$ and Kane was that strained HgTe would be a $3 \mathrm{D}$ TI with the $3 \mathrm{D} Z_{2}$ invariant $(1 ; 000) .{ }^{35)}$ This was experimentally verified in 2011 using MBE-grown films by Brüne et al. ${ }^{129)}$ who reported that a 70 -nm-thick HgTe film grown on a CdTe substrate is uniformly strained $(0.3 \%)$ and obtains a band gap of $\sim 22 \mathrm{meV}$; a strange pattern of quantum Hall effect was observed with Hall plateaus appearing at filling factors $v=2,3,4,5,7,9$. This was interpreted ${ }^{129)}$ to be a result of a combination of two quantum Hall effects occurring in parallel at the top and bottom surfaces that contain slightly different carrier densities. So far, strained $\mathrm{HgTe}$ is unique in that the mobility of the surface carriers can be high enough to present quantum Hall effect.

An interesting material which was experimentally found to be topological without any theoretical prediction is the $(\mathrm{PbSe})_{5}\left(\mathrm{Bi}_{2} \mathrm{Se}_{3}\right)_{3 m}$ compound $(m=1,2) .{ }^{130)}$ This material forms a natural multilayer heterostructure consisting of topological insulator $\left(\mathrm{Bi}_{2} \mathrm{Se}_{3}\right)$ and an ordinary insulator $(\mathrm{PbSe})$ units (Fig. 10). The precise atomic positions within the unit cell has not been determined yet for this material, and hence no $a b$ initio band calculation has been performed. Nakayama et al. found that in this material the $\mathrm{PbSe}$ unit provides a barrier for the electronic states in the $\mathrm{Bi}_{2} \mathrm{Se}_{3}$ unit to be quantum confined, which, for $m=2$, leads to hybridization of the topological interface states at the top and bottom of each $\mathrm{Bi}_{2} \mathrm{Se}_{3}$ unit, resulting in a gapped Dirac-cone structure. Also, the bulk band gap of this $m=2$ compound was found to be as large as $0.5 \mathrm{eV}$, which is probably due to the quantum confinement effect on the bulk band. For $m=1$, the quantum confinement apparently lifts the non-trivial $Z_{2}$ topology from the $\mathrm{Bi}_{2} \mathrm{Se}_{3}$ unit and gives rise to a degenerate parabolic band (Fig. 10).

\subsection{Candidate 3D TIs}

There are other candidate materials of 3D TIs for which the topological surface states have not been observed. There have been many predictions for 3D TIs based on $a b$ initio band calculations, ${ }^{131)}$ but in the following I will discuss only those materials that have been experimentally addressed. 

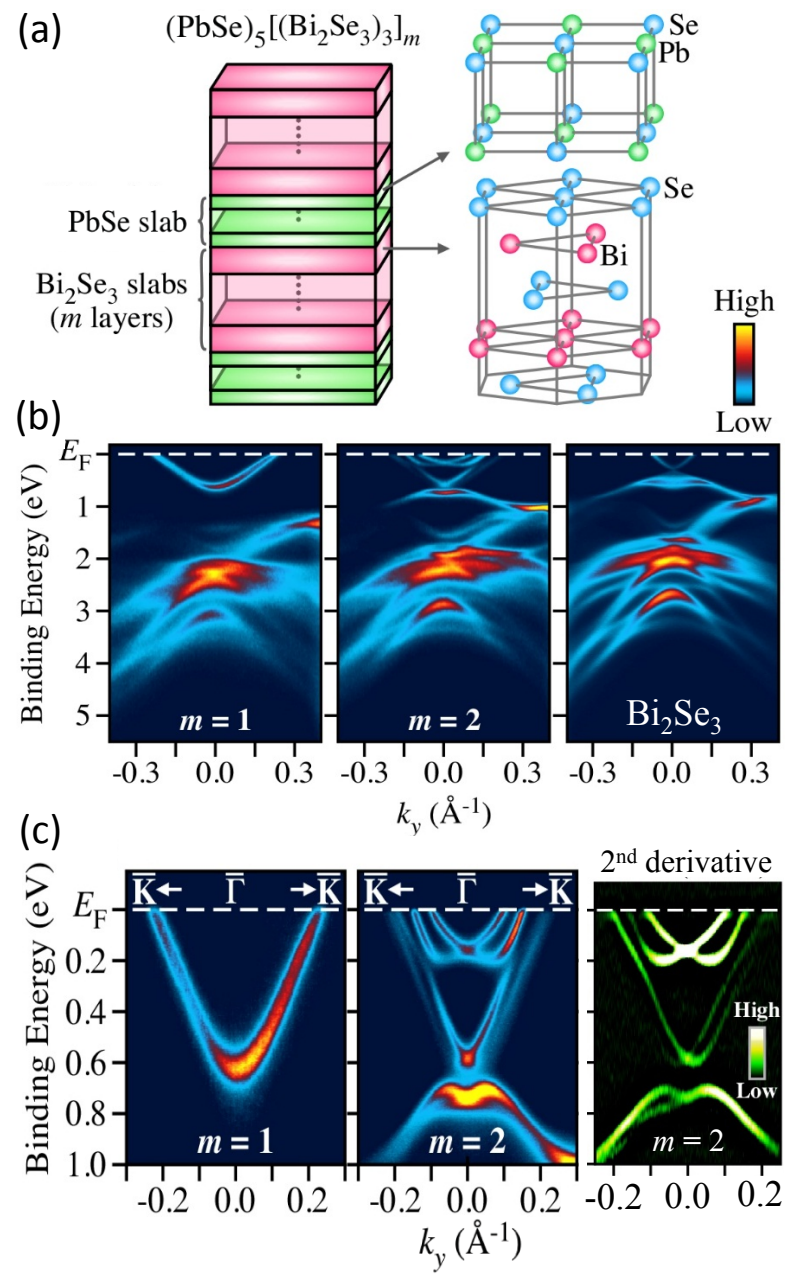

Fig. 10. (Color online) (a) Schematic picture of the crystal structure of $(\mathrm{PbSe})_{5}\left(\mathrm{Bi}_{2} \mathrm{Se}_{3}\right)_{3 m}$, which forms a natural multilayer heterostructure consisting of topological insulator $\left(\mathrm{Bi}_{2} \mathrm{Se}_{3}\right)$ and an ordinary insulator $(\mathrm{PbSe})$ units. (b) ARPES intensities of $(\mathrm{PbSe})_{5}\left(\mathrm{Bi}_{2} \mathrm{Se}_{3}\right)_{3 m}$ for $m=1$ (left) and 2 (center), together with that of $\mathrm{Bi}_{2} \mathrm{Se}_{3}$ (right), plotted as a function of the binding energy and wave vector measured along the $k_{y}$ axis $(\bar{\Gamma}-\bar{K}$ cut $)$ at $T=30 \mathrm{~K}$ with $h v=60 \mathrm{eV}$. (c) Magnified view of the same data for $m=1$ (left) and 2 (center) at low energy; the right panel shows the second derivative of the $m$ $=2$ data, in which the gap opening at the Dirac point is evident. Taken from Ref. 130; copyright American Physical Society (2012).

$\mathrm{Ag}_{2} \mathrm{Te}$ has been known to be a material presenting large, linear-in- $B$ magnetoresistance over an exceedingly wide magnetic-field range, ${ }^{132)}$ and it has been proposed that this unusual property may be associated with its 3D-TI nature. ${ }^{133)}$ ARPES measurements of this material have not been reported, but Aharonov-Bohm (AB) oscillations have been observed in nanowires of $\mathrm{Ag}_{2} \mathrm{Te} .{ }^{134,135)}$ Such an observation suggests the existence of some surface conduction layer, but its topological nature is still left to be confirmed, because similar $\mathrm{AB}$ oscillations are known to originate from trivial surface states in InN nanowires. ${ }^{136)}$

A very interesting candidate is $\mathrm{SmB}_{6}$, which may be a topo- logical Kondo insulator, ${ }^{137-139)}$ because the band bap of this material stems from Kondo effect. If this material is indeed a $\mathrm{TI}$, it will be the first material where electron correlations and nontrivial band topology both play important roles. So far, strong evidence for surface-dominated transport at low temperature has been reported, ${ }^{140-142)}$ but existing ARPES data do not resolve topological surface state within the small gap $(\sim 20 \mathrm{meV}) .{ }^{143)}$ A puzzling feature in the reported surfacetransport data ${ }^{142)}$ is that the observed surface carrier density of $1.1 \times 10^{15} \mathrm{~cm}^{-2}$ is about 1000 times larger than that found in other TI materials and is obviously too large for the expected Dirac carrier density. ${ }^{139)}$ One should keep in mind that the surface-dominated transport can be observed in insulators in ambient condition due to the formation of a trivial accumulation (or inversion) layer, as reported for pure Te by von Klitzing and Landwehr, ${ }^{144)}$ and hence such an observation alone does not give evidence for a TI phase.

Another interesting candidate is $\mathrm{Bi}_{14} \mathrm{Rh}_{3} \mathrm{I}_{9}$, which may be a weak 3D TI with the $Z_{2}$ index $(0 ; 001) .{ }^{145)}$ The crystal structure of this material can be viewed as a stack of intermetallic $\left[\left(\mathrm{RhBi}_{4}\right)_{3} \mathrm{I}\right]^{2+}$ layers and intervening $\left[\mathrm{Bi}_{2} \mathrm{I}_{8}\right]^{2-}$ zigzag-chain layers; the former consist of graphene-like honeycomb network of edge-sharing $\mathrm{RhBi}_{8}$ cubes, giving rise to an electronic structure which is similar to that in graphene but is enriched by strong SOC due to $\mathrm{Bi}$. The $a b$ initio band calculations predict that this is a quasi-2D system which essentially consists of a stacked 2D TI layers; as a result, the system is predicted to be a weak 3D TI with the topological surface state expected only on the sides of the stacks. The bulk band structure seen by ARPES is in reasonable agreement with the calculations, but no surface state was detected because the ARPES was done on the cleaved top surface where the surface state is not expected. Obviously, some transport measurements to detect the topological surface state on the side surface is necessary to elucidate the TI nature of this material.

\subsection{Topological semimetal}

The term "topological semimetal" can be used for three different classes of materials. The first is an ordinary semimetal possessing separate electron and hole pockets compensating each other, but the parity eigenvalues of the valence band gives rise to a nontrivial $Z_{2}$ topology; an example is pure $\mathrm{Sb}$, whose topology is characterized by the $3 \mathrm{D} Z_{2}$ invariant $(1 ; 111) .{ }^{35,39)}$ The second is a zero-gap semiconductor which possesses band inversion, such as unstrained HgTe; this class of materials can be made genuine TIs by lowering the crystal symmetry to open a gap. The third is called Weyl semimetal, ${ }^{146)}$ which is probably the most fundamentally interesting. In the following, I will discuss the latter two classes.

Interesting examples of the candidate topological zero-gap semimetals are Heusler or half-Heusler compounds. ${ }^{131)}$ They are ternary intermetallic compounds with $X_{2} Y Z$ (Heusler) or $X Y Z$ (half-Heusler) compositions. Their crystal structures consist of basic $Y Z$ sublattice (which takes the zinc-blende 
structure similar to $\mathrm{HgTe}$ ) stuffed with $\mathrm{X}$ in the void space. Many Heusler or half-Heusler compounds were predicted to have band inversion and be topological based on ab initio band calculations; ${ }^{147-151)}$ under uniaxial pressure which lowers the crystal symmetry, they obtain a gap and are expected to become TIs. The ARPES experiments done on $R \mathrm{PtBi}(R=\mathrm{Lu}$, Dy, Gd) found metallic surface states distinct from the bulk bands, but the surface-band structure does not appear to support a topological origin. ${ }^{152)}$ Interestingly, superconductivity has been found in $\mathrm{LaPtBi}^{153)}$ and $\mathrm{YPtBi}^{154)}$ if those materials are indeed topological, the fate of the surface states in the superconducting state is an intriguing issue.

The idea of Weyl semimetal was theoretically proposed by Wan et al. in 2011.146) Weyl fermions have definite chirality, which protects them from gapping, and they are described by a massless two-component Dirac equation. In solids, when TRS is broken in an inversion symmetric system and nondegenerate valence and conduction bands touch at an accidental degeneracy point in a 3D BZ, the low-energy physics is approximated by massless two-component Dirac equation and electrons obey the Weyl Hamiltonian $H= \pm \hbar v_{F} \boldsymbol{\sigma} \cdot \mathbf{k}=$ $\pm \hbar v_{F}\left(k_{x} \sigma_{x}+k_{y} \sigma_{y}+k_{z} \sigma_{z}\right)$. By symmetry, such a degeneracy point (Weyl point) must come in pairs, and Wan et al. showed that a pair of Weyl points give rise to an arc of zero-energy excitation (Fermi arc) to connect them in the projected surface BZ. ${ }^{146)}$ The appearance of this gapless state on the surface of Weyl semimetal can be viewed as a result of the bulkboundary correspondence to signify the non-trivial topology. ${ }^{155,156)}$ It was proposed based on ab initio band calculations that pyrochlore iridates such as $\mathrm{Y}_{2} \mathrm{Ir}_{2} \mathrm{O}_{7}$ in the antiferromagnetic phase may realize such a Weyl semimetal. ${ }^{146,157)}$ In this context, a recent study of $\mathrm{Nd}_{2}\left(\mathrm{Ir}_{1-x} \mathrm{Rh}_{x}\right)_{2} \mathrm{O}_{7}$ found a transition from a correlated metal to a Mott insulator with decreasing $x$ that is accompanied by a gradual reduction of the spectral weight, and it is possible that the Weyl semimetal phase exists at the critical point. ${ }^{158)}$

The Weyl semimetal phase is also conceivable in a TR symmetric system with broken inversion symmetry, and it was proposed that such a phase may be achieved by breaking the inversion symmetry through elaborate multilayer structures using $\mathrm{HgTe} / \mathrm{CdTe}^{159)}$ or the $\mathrm{TlBi}\left(\mathrm{S}_{1-x} \mathrm{Se}_{x}\right)_{2}$ system at the topological phase transition point. ${ }^{160)}$

\subsection{Topological crystalline insulator}

We have already seen that band insulators can be topologically classified by evaluating the $Z_{2}$ invariant from valenceband Bloch wave functions. This classification is based on TRS of the system. It turned out that this is not the only possible topological classification of band insulators, but it is also possible to classify band insulators based on topologies protected by point-group symmetries of the crystal lattice. ${ }^{161)}$ Those insulators that have nontrivial topology protected by point-group symmetries are called topological crystalline insulators (TCIs). ${ }^{161)}$ So far, concrete topological invariants are elucidated for systems possessing four-fold $\left(C_{4}\right)$ or six-fold $\left(C_{6}\right)$ rotation symmetry ${ }^{161)}$ and also for systems with mirror symmetry. ${ }^{61)}$ In particular, the latter case gained significant attention after the prediction of a concrete example, SnTe, was made by Hsieh et al. ${ }^{61)}$

In TCIs with mirror symmetry, the topology is specified by the topological invariant $n_{\mathcal{M}}$ called mirror Chern number, which evaluates the Chern number in only one of the two
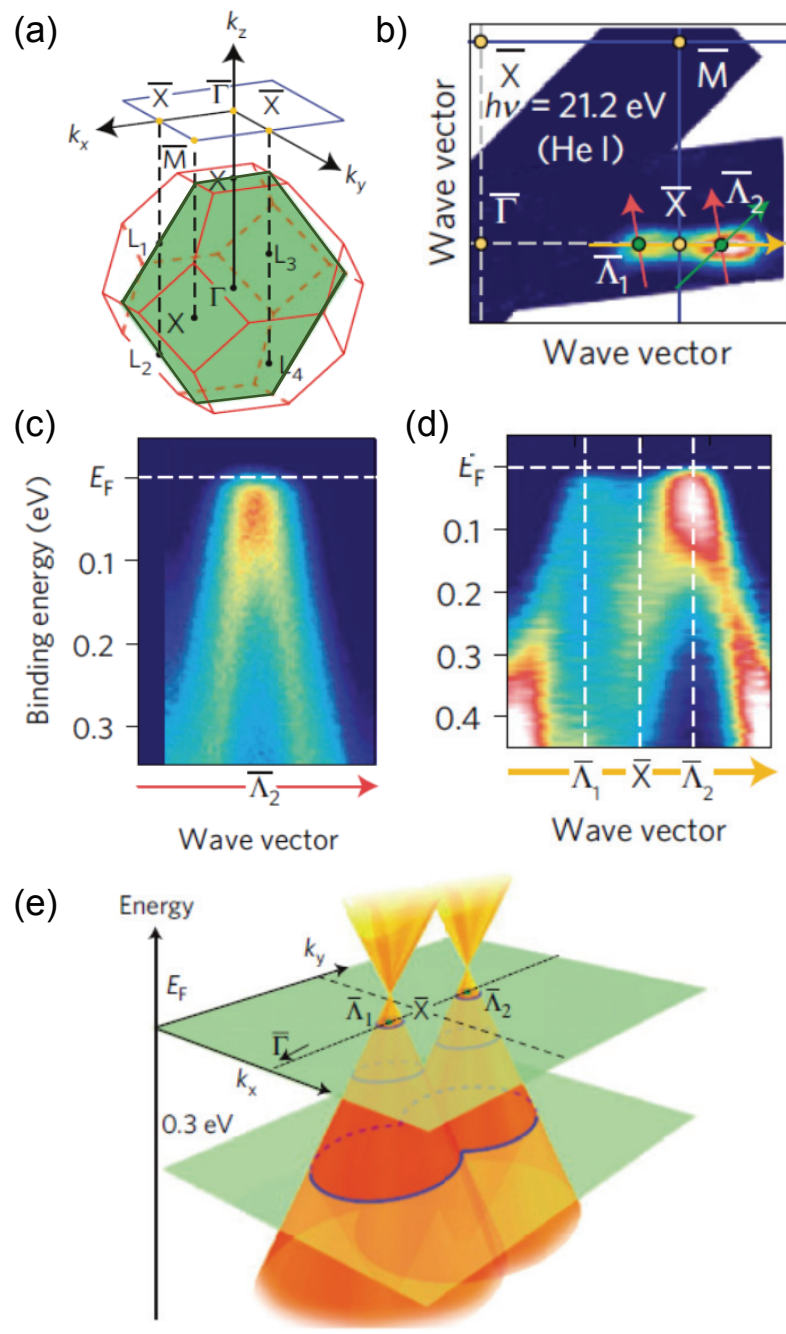

Fig. 11. (Color online) ARPES data on cleaved (001) surface of SnTe. (a) The bulk BZ and the corresponding (001) surface BZ of SnTe; $\Gamma, L, X$ are the symmetry points in the bulk $3 \mathrm{D} \mathrm{BZ}$, whereas $\bar{\Gamma}, \bar{X}, \bar{M}$ are in the surface 2D BZ. The (110) mirror plane is indicated by the shaded area. Note that two $L$ points [e.g. $L_{1}$ and $L_{2}$ in panel (a)] are projected to the same $\bar{X}$ point. (b) ARPES intensity mapping in the surface BZ at $E_{F}$ measured with $h v=21.2$ $\mathrm{eV}$ at $30 \mathrm{~K}$. (c) Near- $E_{F}$ ARPES intensity as a function of the wave vector and the binding energy measured along the cut which is nearly parallel to the $\bar{X}-\bar{M}$ direction and is crossing the $\bar{\Lambda}_{2}$ point [one of the red arrows in panel (b)]. (d) Near- $E_{F}$ ARPES intensity along the $\bar{\Gamma}-\bar{X}$ cut [yellow arrow in panel (b)]. (e) Schematic 2D band dispersions near $E_{F}$ concluded from the data in panels (c) and (d) depicting the characteristic double Dirac-cone structure. Taken from Ref. 62. 
Hilbert subspaces divided according to the mirror eigenvalues. ${ }^{88)}$ Based on ab initio calculations, Hsieh et al. showed that SnTe has band inversions at four TRIMs (four equivalent $L$ points) in the $3 \mathrm{D} \mathrm{BZ},{ }^{61)}$ which gives rise to a nontrivial mirror Chern number $n_{\mathcal{M}}=-2$, while its $Z_{2}$ invariant is trivial, $(0 ; 000)$. They further predicted that the surface state on the $\{001\}$ surface should present a peculiar double-Dirac-cone structure near the $\bar{X}$ points, because two $L$ points are projected onto the same $\bar{X}$ point [Fig. 11(a)]. ${ }^{61)}$

It is interesting that the experimental verification of this TCI prediction showcases how severe the competitions in the TI research are. The prediction ${ }^{61)}$ was posted to the preprint server in February 2012. In June, three preprints reporting experimental discovery of a TCI were posted to the preprint server in about two weeks, and two of them were published on the same day in September 2012 in different journals (Nature Physics $^{62)}$ and Nature Materials ${ }^{162)}$ ), while the other one ${ }^{163)}$ reported by a group at Princeton University was not published; the Princeton group performed new measurements, and a paper containing new data were submitted in August and published in November. ${ }^{164)}$

Among the first two published papers, the one by Tanaka et $a l{ }^{62)}$ reported straightforward confirmation of the prediction in SnTe, and the observed surface state with the double Diraccone structure [Fig. 11] was in good qualitative agreement with the theory; they also showed that the cousin material $\mathrm{PbTe}$ does not present any surface state. The other one by Dziawa et al. ${ }^{162)}$ reported the TCI phase in $\mathrm{Pb}_{0.77} \mathrm{Sn}_{0.23} \mathrm{Se}$, which shows a transition to a trivial phase upon increasing the temperature. The work by $\mathrm{Xu}$ et al. published later ${ }^{164)}$ reported a TCI phase in $\mathrm{Pb}_{0.6} \mathrm{Sn}_{0.4} \mathrm{Te}$ and a trivial phase in $\mathrm{Pb}_{0.8} \mathrm{Sn}_{0.2} \mathrm{Te}$, together with spin-resolved ARPES data showing helical polarization on each of the double Dirac cones. A more recent paper by Tanaka et al. ${ }^{165)}$ nailed down that the topological phase transition in $\mathrm{Pb}_{1-x} \mathrm{Sn}_{x}$ Te occurs at $x_{c} \simeq 0.25$; furthermore, they found that the separation between the two Dirac cones near the $\bar{X}$ points systematically narrows when $x$ is reduced toward $x_{c}$, but they never merge before the transition eliminates them.

In passing, the mirror Chern number $n_{\mathcal{M}}$ can also be used for TR-invariant 3D TIs to further classify them. ${ }^{88)}$ For example, $\mathrm{Bi}_{1-x} \mathrm{Sb}_{x}$ is a TI with $Z_{2}$ invariant $(1 ; 111)$, and it can have $n_{\mathcal{M}}= \pm 1$. The sign of $n_{\mathcal{M}}$ is called mirror chirality, which is related to the sign of the $g$ factor. The first experimental work that addressed this additional topological property in a TI was the spin-resolved ARPES done by Nishide et al., ${ }^{40)}$ who elucidated that the mirror chirality is -1 in $\mathrm{Bi}_{1-x} \mathrm{Sb}_{x}$.

The discovery of TCIs significantly widened the scope of topological materials. Already, detailed topological classification schemes for all point-group symmetries have been proposed, ${ }^{166)}$ and also the mirror topology has been expanded to superconductors. ${ }^{167-169)}$ Experimentally, elucidating the interplay between $Z_{2}$ topology and mirror topology in materials like SnTe under uniaxial strain would be an interesting issue.

\section{How to Confirm TI Materials}

In this section, I briefly summarize the possible experimental procedures to confirm whether a material is a TI or not. In the case of 2D TIs, one needs to probe the existence of helical $1 \mathrm{D}$ edge state, which is possible only through quantum transport experiments using nano-fabricated device structures. The existence of the edge state can be seen through conductance quantization in the insulating regime. ${ }^{31)}$ Also, the helical spin polarization of the edge state may be detected by transport experiments using spin Hall effect. ${ }^{70)}$

For 3D TIs, the simplest and the most convincing is to observe the Dirac cone by ARPES experiments. To firm up the identification of a TI, one should employ spin-resolved ARPES to confirm that the Dirac cone is non-degenerate and is helically spin polarized. ${ }^{39,40)}$

Unfortunately, not all materials are suitable for ARPES, which requires clean and flat surface that is usually obtained by cleaving single crystals. When single crystals are not available or the material does not cleave well, APRES becomes difficult. In such a case, one may rely on transport experiments. Ideally, if the bulk is sufficiently insulating and the surface carriers have high enough mobility, one would be able to confirm that the transport is occurring through the surface and that the surface carriers are Dirac fermions. The former can be done by looking at the sample-size dependence of the conductance, ${ }^{108,140-142)}$ and the latter may be accomplished by elucidating the $\pi$ Berry phase in the quantum oscillations from the surface state. ${ }^{103,104,108,170-173)}$ (Detailed discussions on the identification of the $\pi$ Berry phase will be given in Sec. 8.3.) It should be emphasized that confirming the Diracfermion nature of the surface carriers is important, because trivial accumulation layer or inversion layer that may form on the surface of an insulator may also give rise to surfacedominated transport. ${ }^{144)}$

The Dirac-fermion nature may also be confirmed by STS experiments in magnetic fields, because massless Dirac fermions present peculiar Landau quantization in which the level spacing changes as $\sqrt{N}$ and the zero-energy Landau level is pinned to the Dirac point (see detailed discussions in Sec. 8.2); by looking at the bias-voltage dependence of the Landau quantization peaks, one can identify Dirac fermions. ${ }^{97,174,175)}$ Similarly, magneto-optics experiments to detect the Landau level transitions can be used for detecting the peculiar quantization scheme to identify Dirac fermions on the surface. ${ }^{91)}$

One should note that in reality, it is often very difficult to obtain sufficiently bulk-insulating samples of a candidate material. In that case, transport measurements are not very useful. If it is possible to detect the equilibrium spin current (which is carried only by the helical Dirac fermions and hence is not bothered by bulk carriers) by some electromagnetic means, ${ }^{176)}$ it would become a very useful tool for identifying a TI. However, feasibility of such an experiment is not clear at the moment. 


\section{Syntheses of TI Materials}

\subsection{Bulk single crystals}

Except for $\mathrm{Bi}_{1-x} \mathrm{Sb}_{x}$, all the confirmed 3D TI materials are chalcogenides (i.e. compounds containing chalcogen atoms $\mathrm{S}$, $\mathrm{Se}$, and $\mathrm{Te}$ ). Since chalcogen atoms are volatile, the syntheses of chalcogenides are done in sealed evacuated quartz-glass tubes, which can sustain temperatures up to $1000^{\circ} \mathrm{C}$. Such a necessity of containment limits the range of applicable growth techniques, and one usually uses the Bridgman method. In this method, the temperature of the melt is gradually reduced while keeping a temperature gradient in the tube, so that the crystallization starts at the cold end and the crystal grows as the solidification proceeds from this end. Most of the popular TI materials including $\mathrm{Bi}_{2} \mathrm{Se}_{3}, \mathrm{Bi}_{2} \mathrm{Te}_{3}$, and $\mathrm{Bi}_{2} \mathrm{Te}_{2} \mathrm{Se}$ are grown by the Bridgman method.

Another possible crystal growth technique is the vapor transport. In this technique, one puts a chunk of polycrystalline material on one end of a sealed quartz-glass tube. The tube is kept for a long time in a furnace with a certain temperature gradient, in which the polycrystalline material is on the hotter end. The temperature gradient is chosen so that the material sublimates at the hotter end and crystalizes at somewhere in a colder part. When some reagent such as $I_{2}$ is used as a transporter, the technique is called chemical vapor transport; if the material easily sublimates, one does not need a transporter and the technique is called physical vapor transport. Single crystals of TCI materials, SnTe, $(\mathrm{Pb}, \mathrm{Sn}) \mathrm{Se}$, and $(\mathrm{Pb}, \mathrm{Sn}) \mathrm{Te}$ are usually grown with a vapor transport technique.

Since the tetradymite compounds cleave easily, they can be made into a very thin (down to only a few nm thick) flakes by employing an exfoliation technique similar to that used for making graphene samples using Scotch tapes. Such thin flakes are particularly useful for experiments involving gating to electrostatically control the surface chemical potential. ${ }^{177,178)}$

\subsection{Thin films}

For the growth of high-quality epitaxial thin films of TI materials, molecular beam epitaxy (MBE) technique is usually employed. ${ }^{179)}$ So far, reports of MBE growths have been made for $\mathrm{Bi}_{1-x} \mathrm{Sb}_{x}{ }^{180)}$ or relatively simple tetradymite compounds $\mathrm{Bi}_{2} \mathrm{Se}_{3},{ }^{173,174,181-191)} \mathrm{Bi}_{2} \mathrm{Te}_{3},{ }^{192)}$ and $\mathrm{Sb}_{2} \mathrm{Te}_{3},{ }^{97)}$ as well as their solid-solutions such as $(\mathrm{Bi}, \mathrm{Sb})_{2} \mathrm{Te}_{3} .{ }^{193)}$ Those materials can be grown by co-evaporating the constituent elements with suitable flux ratios. Chemical vapor deposition (CVD) technique has also been applied to the growth of $\mathrm{Bi}_{2} \mathrm{Se}_{3},{ }^{194}$ ) but the reported film quality has not been as good as the best MBE-grown films.

For epitaxial growths of thin films, the lattice matching between the substrate and the grown material is usually very important. However, in the case of tetradymite TI materials, thanks to the existence of the van der Waals gap between the QLs, the lattice matching with the substrate is not crucial and the epitaxial growth proceeds in the so-called van der Waals epitaxy mode, ${ }^{195)}$ in which the substrate and the films are only weakly bonded with the van der Waals force and hence the lattice matching condition is greatly relaxed. In particular, epitaxial growths of $\mathrm{Bi}_{2} \mathrm{Se}_{3}$ have been reported for various substrates including $\mathrm{Si}(111){ }^{181-184)}$ graphene-terminated $6 \mathrm{H}-\mathrm{SiC}(0001),{ }^{174,185)} \mathrm{SrTiO}_{3}(111),{ }^{186)} \mathrm{GaAs}(111),{ }^{187)}$ sapphire(0001), ${ }^{173,188,189)} \mathrm{CdS}(0001),{ }^{190)}$ and $\operatorname{InP}(111) .{ }^{191)}$

It turned out that the control of the substrate temperature is the most crucial ingredient for obtaining high-quality films of $\mathrm{Bi}_{2} \mathrm{Se}_{3}$ with a large area of atomically flat terraces. ${ }^{189)}$ In particular, a two-step method in which the depositions of the first layer and subsequent layers are done at different temperatures, to promote initial adhesion and crystallization separately, has been reported to yield best quality films. ${ }^{173,182,183,188,189)}$

For the TCI material SnTe, a technique called hot-wall epitaxy has been used in the past, ${ }^{196)}$ yielding good quality samples with a reasonably high mobility $\left(\sim 2700 \mathrm{~cm}^{2} / \mathrm{Vs}\right)$. For $\mathrm{SnTe}$ which is a cubic material with rock-salt structure, good lattice matching is crucial for epitaxial growth. $\mathrm{BaF}_{2}$ has been traditionally used as a substrate, ${ }^{196)}$ but recently, using $\mathrm{Bi}_{2} \mathrm{Te}_{3}$ as a buffer layer between SnTe and sapphire was reported to yield high quality films that present surface $\mathrm{SdH}$ oscillations. ${ }^{197)}$

\subsection{Nanoribbons and nanoplates}

Mesoscopic transport experiments of $\mathrm{Bi}_{2} \mathrm{Se}_{3},{ }^{198)}$ $\mathrm{Bi}_{2} \mathrm{Te}_{3},{ }^{199,200)}$ and $\mathrm{Bi}_{2} \mathrm{Te}_{2} \mathrm{Se}^{201)}$ have been performed using nanoribbons and nanoplates. $\mathrm{Bi}_{2} \mathrm{Se}_{3}$ nanoribbons are usually synthesized by gold-catalyzed vapor liquid solid (VLS) technique. ${ }^{198)}$ Typically, $\mathrm{Bi}_{2} \mathrm{Se}_{3}$ powder is placed in the center of a tube furnace through which Ar gas flows and transports evaporated $\mathrm{Bi}_{2} \mathrm{Se}_{3}$; a silicon substrate decorated with $\mathrm{Au}$ nanoparticles are placed downstream in the tube furnace, and the growth of nanoribbons proceeds as $\mathrm{Au}$ nanoparticles absorb $\mathrm{Bi}_{2} \mathrm{Se}_{3}$ vapor and leave crystallized $\mathrm{Bi}_{2} \mathrm{Se}_{3}$ nanoribbons beneath them. Naturally, the diameter of grown nanoribbons is roughly determined by the size of the $\mathrm{Au}$ nanoparticles (typically $\sim 20-\mathrm{nm}$ diameter). When there are no Au nanoparticles to work as catalyst, nanoplates (typically a few nm thick and a few $\mu \mathrm{m}$ wide) are obtained instead of nanoribbons. ${ }^{199,201)}$

\section{Defect Chemistry and Engineering}

An important theme in the research of TI materials is to reduce unintentionally-doped bulk carriers that hinder observations of surface transport properties. Only in the case of HgTe thin films, which can be grown in very high purity using an MBE technique, unwanted bulk carriers are not an issue. In most other materials, one needs to find suitable ways to reduce bulk carriers. In this section, I will use the KrögerVink notation to describe defects in crystals; for example, $V_{\mathrm{Se}}^{\bullet \bullet}$ means a selenium vacancy with double positive charge, and $\mathrm{Bi}_{\mathrm{Te}}^{\prime}$ means a bismuth ion sitting on the tellurium lattice site with a single negative charge.

Naturally-grown $\mathrm{Bi}_{2} \mathrm{Se}_{3}$ crystals are always electron doped, 
with the typical bulk carrier density $n_{3 \mathrm{D}}$ of $10^{19} \mathrm{~cm}^{-3},{ }^{202-204)}$ because of the thermodynamically inevitable $\mathrm{Se}$ vacancies $\left(V_{\mathrm{Se}}^{\bullet \bullet}\right)$ or $\mathrm{Se}$ anti-site defects $\left(\mathrm{Se}_{\mathrm{Bi}}^{\bullet}\right)$ that have low formation energies. ${ }^{205,206)}$ Doping $\mathrm{Ca}^{2+}$ to the $\mathrm{Bi}^{3+}$ site has been reported to be useful for reducing $n$-type carriers and eventually achieving $n$-to- $p$ type transition. ${ }^{207)}$ However, Ca doping obviously introduces strong scattering centers and the electron mobility becomes low in Ca-doped $\mathrm{Bi}_{2} \mathrm{Se}_{3} .{ }^{207}$ ) Optimization of the growth condition ${ }^{202)}$ and isovalent $\mathrm{Sb}$ doping to the Bi site ${ }^{170,203)}$ are both reported to be useful for reducing $n_{3 \mathrm{D}}$ down to $10^{16} \mathrm{~cm}^{-3}$ while keeping a high electron mobility that allowed observation of surface $\mathrm{SdH}$ oscillations, ${ }^{170)}$ although $n$-to- $p$ type transition was not achieved with these routes. Recently, Ren et al. ${ }^{208)}$ succeeded in growing high-mobility $p$ type crystals of $\mathrm{Bi}_{2} \mathrm{Se}_{3}$ by combining Cd doping and a Se-rich growth condition; furthermore, subsequent annealing of such $p$-type crystals to compensate for the $p$-type carriers by the electrons coming from Se vacancies made it possible to obtain both $n$ - and $p$-type samples with low bulk carrier density, in which surface $\mathrm{SdH}$ oscillations were observable. ${ }^{208)}$

In contrast to $\mathrm{Bi}_{2} \mathrm{Se}_{3}, \mathrm{Bi}_{2} \mathrm{Te}_{3}$ can be grown in both $n$ - and p-types. ${ }^{104)}$ This is because Te anti-site defects $\left(\mathrm{Te}_{\mathrm{Bi}}^{\bullet}\right)$ or $\mathrm{Bi}$ anti-site defects $\left(\mathrm{Bi}_{\mathrm{Te}}^{\prime}\right)$ are formed in Te-rich or Bi-rich conditions, respectively, and $\mathrm{Te}_{\mathrm{Bi}}^{\bullet}$ is a donor while $\mathrm{Bi}_{\mathrm{Te}}^{\prime}$ is an acceptor. ${ }^{205,206)}$ Nevertheless, it is difficult to obtain $\mathrm{Bi}_{2} \mathrm{Te}_{3}$ samples with low $n_{3 \mathrm{D}}$. By growing a boule of crystal with a compositional gradient, $\mathrm{Qu}$ et al. were able to pick up samples with $n_{3 \mathrm{D}} \approx 10^{16} \mathrm{~cm}^{-3}$ in which they observed surface SdH oscillations. $^{104)}$

The $\mathrm{Bi}_{2} \mathrm{Te}_{2} \mathrm{Se}$ compound can be much more insulating than $\mathrm{Bi}_{2} \mathrm{Se}_{3}$ or $\mathrm{Bi}_{2} \mathrm{Te}_{3}$ if grown in a slightly Se-rich condition. ${ }^{103}$ ) This is because $\mathrm{Bi}_{2} \mathrm{Te}_{2} \mathrm{Se}$ naturally crystallize in a chalcogenordered structure with Te-Bi-Se-Bi-Te QL units (Fig. 7). ${ }^{209)}$ This peculiar structure solves the problems of Se-vacancies in $\mathrm{Bi}_{2} \mathrm{Se}_{3}$ and $\mathrm{Bi} / \mathrm{Te}$ antisite defects in $\mathrm{Bi}_{2} \mathrm{Te}_{3}$ at the same time: First, because $\mathrm{Se}$ is concealed in the middle of the QL, Se vacancy is much more difficult to occur than in $\mathrm{Bi}_{2} \mathrm{Se}_{3}$. Second, since the electronegativity of $\mathrm{Se}$ is stronger than that of $\mathrm{Te}, \mathrm{Bi}$ is more strongly bonded to $\mathrm{Se}$ than to $\mathrm{Te}$, which discourages the occurrence of $\mathrm{Bi} / \mathrm{Te}$ antisite defects. As a result, $\mathrm{Bi}_{2} \mathrm{Te}_{2} \mathrm{Se}$ can be reasonably bulk-insulating. In addition, its ordered structure ensures a high mobility, and consequently the topological surface state can be easily studied through transport properties of this material. ${ }^{103,105)}$ In passing, materials with similar chalcogen-ordered structure, $\mathrm{Bi}_{2} \mathrm{Te}_{1.6} \mathrm{~S}_{1.4},{ }^{210}$ ) $\mathrm{Bi}_{1.1} \mathrm{Sb}_{0.9} \mathrm{Te}_{2} \mathrm{~S},{ }^{210}$ ) and $\mathrm{Bi}_{2}(\mathrm{Te}, \mathrm{Se})_{2}(\mathrm{Se}, \mathrm{S})$ (natural Kawazulite mineral $)^{211)}$ have been recently reported, and the latter two are found to show moderately bulk-insulating behavior.

Importantly, even higher bulk-insulating properties than in $\mathrm{Bi}_{2} \mathrm{Te}_{2} \mathrm{Se}$ have been achieved by utilizing a solid solution $\mathrm{Bi}_{2-x} \mathrm{Sb}_{x} \mathrm{Te}_{3-y} \mathrm{Se}_{y}(y \geq 1) .{ }^{107,108)}$ In this material, the middle of the QL is preferentially occupied by $\mathrm{Se}$, hence retaining the essential virtue of $\mathrm{Bi}_{2} \mathrm{Te}_{2} \mathrm{Se}$. In addition, the $\mathrm{Bi} / \mathrm{Sb}$ ratio in the cation layers and the Te/Se ratio in the outer layers af-
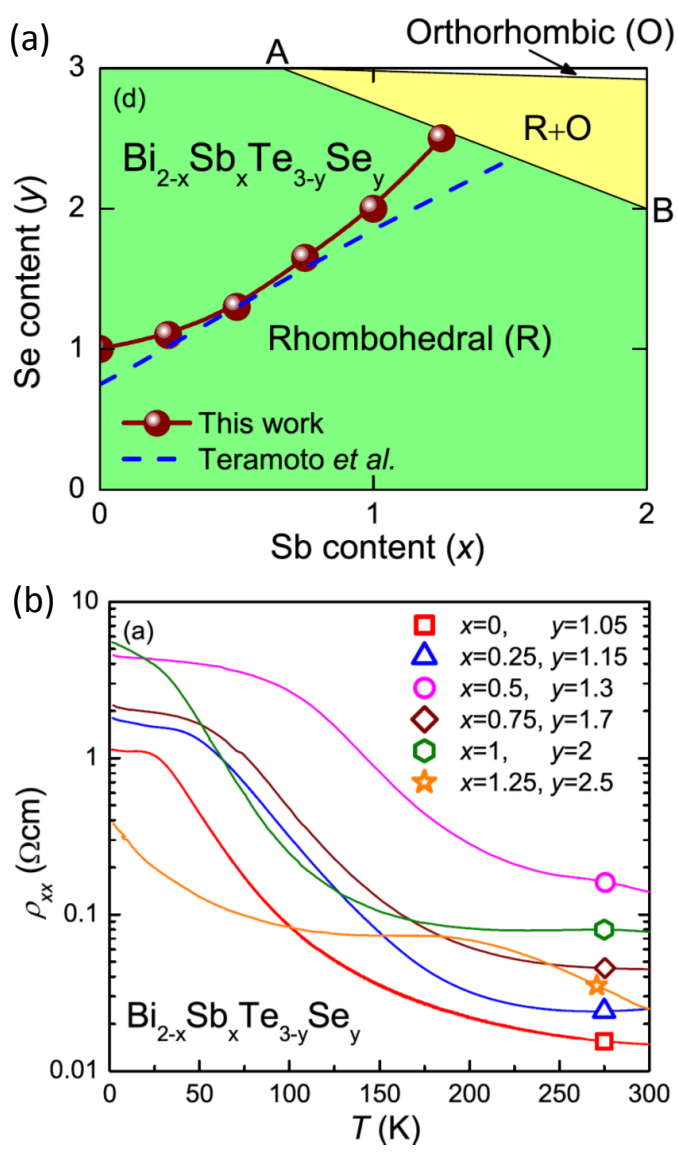

Fig. 12. (Color online) (a) Composition-structural phase diagram of the $\mathrm{Bi}_{2-x} \mathrm{Sb}_{x} \mathrm{Te}_{3-y} \mathrm{Se}_{y}$ system. The circles denote the compositions where the compensation between $n$ - and $p$-type carriers are maximally achieved. The dashed line denotes the insulating composition previously suggested by Teramoto and Takayanagi [J. Phys. Chem. Solids 19 (1961) 124]. (b) Temperature dependencies of $\rho_{x x}$ for the series of BSTS samples at optimized compositions. Note that the vertical axis is in a logarithmic scale. Taken from Ref. 107; copyright American Physical Society (2011).

fect the levels of acceptors and donors, respectively, making it possible to achieve a maximally compensated situation. Indeed, it was found that a reasonably bulk-insulating behavior is observed for a series of compositions $(x, y)$ which forms a curved line in the $x$ vs $y$ phase diagram (Fig. 12). Furthermore, the surface Dirac-cone structure of $\mathrm{Bi}_{2-x} \mathrm{Sb}_{x} \mathrm{Te}_{3-y} \mathrm{Se}_{y}$ was found to present a systematic change along the insulating composition line (Fig. 13), which makes it possible to tune the properties of the surface Dirac fermions. ${ }^{212)}$ Such a tuning of the Dirac-carrier properties has been dubbed "Dirac-cone engineering". In particular, one can obtain both $p$ - and $n$-type Dirac carriers by tuning $(x, y)$ along the insulating line, which is useful for designing a $p-n$ junction of the topological surface state. In this respect, similar band engineering has also been achieved in $(\mathrm{Bi}, \mathrm{Sb})_{2} \mathrm{Te}_{3} .{ }^{193)}$

In fact, another useful approach to reducing bulk carriers is to make a solid solution of $\mathrm{Bi}_{2} \mathrm{Te}_{3}$ and $\mathrm{Sb}_{2} \mathrm{Te}_{3}$. Growths 

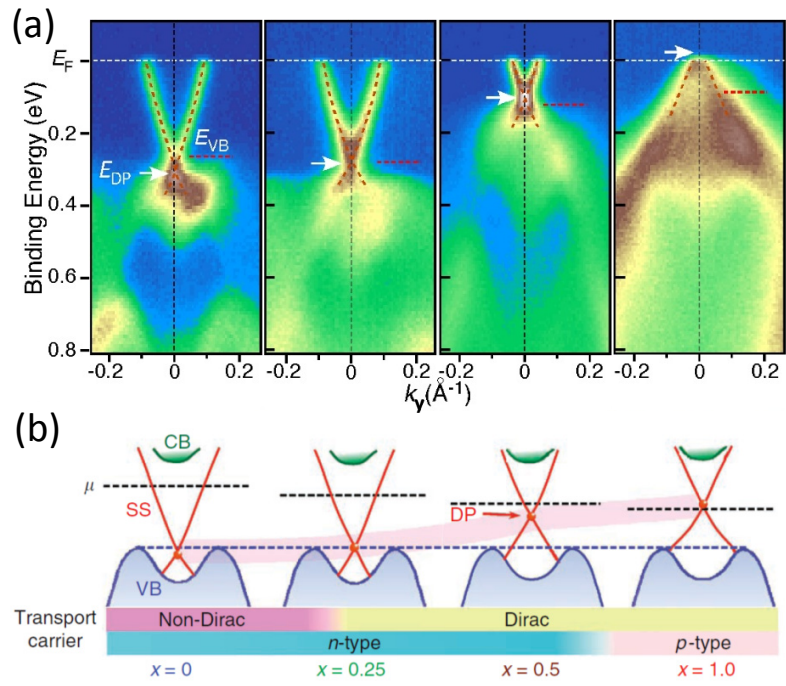

Fig. 13. (Color online) (a) ARPES intensities of $\mathrm{Bi}_{2-x} \mathrm{Sb}_{x} \mathrm{Te}_{3-y} \mathrm{Se}_{y}$ for, from left to right, $(x, y)=(0,1),(0.25,1.15),(0.5,1.3)$, and $(1,2)$, measured with $h v=58 \mathrm{eV}$ along the $k_{y}$ axis $(\bar{\Gamma}-\bar{K}$ cut $)$ at $T=30 \mathrm{~K}$. White arrows and thick (red) dashed lines indicate the energy positions of the Dirac point $\left(E_{\mathrm{DP}}\right)$ and the valence band top $\left(E_{\mathrm{VB}}\right)$, respectively. (b) Schematic band diagrams for $\mathrm{Bi}_{2-x} \mathrm{Sb}_{x} \mathrm{Te}_{3-y} \mathrm{Se}_{y}$ at the insulating compositions, derived from the data shown in (a). Taken from Ref. 212.

of thin films ${ }^{193)}$ and nanoplates ${ }^{213)}$ of $(\mathrm{Bi}, \mathrm{Sb})_{2} \mathrm{Te}_{3}$ as well as nanoribbons ${ }^{214)}$ of $(\mathrm{Bi}, \mathrm{Sb})_{2} \mathrm{Se}_{3}$ have been reported, and in those samples the chemical potential was successfully tuned to be close to the Dirac point in the middle of the band gap.

\section{Properties of 3D TI Materials}

\subsection{Surface state and helical spin polarization}

The most prominent property of a TI is the existence of a gapless surface state. The gapless nature is protected by TRS in $Z_{2}$ topological insulators. What makes this surface state distinct from ordinary surface states (including accumulation and inversion layers) is its helical spin polarization (Fig. 1), which is also called spin-momentum locking; namely, the surface state is spin non-degenerate and the direction of the spin is perpendicular to the momentum vector and is primarily confined in the surface plane. In fact, if a band has such a peculiar spin polarization and the system preserves TRS, there must be a Kramers partner for each eigenstate and Kramers theorem dictates that the two eigenstates cross each other at TRIMs, which guarantees the gapless nature of the surface state [see Fig. 6(b)].

The helical spin polarization of the surface state means that a dissipationless spin current exists on the surface in equilibrium, because there is no net charge flow but the spin angular momentum flows in the direction perpendicular to the spin direction. The spin helicity of the surface state (i.e. whether the up spin is associated with $+k$ or $-k$ ) determines the spin current direction. In all the cases tested so far, the spin helicity has been found to be left-handed [i.e. the spin points to $-y$ direction for $\mathbf{k}=(+k, 0)$ ] when $E_{F}$ is above the Dirac point, and it becomes right-handed for $E_{F}$ below the Dirac point [see Fig. 1(d)].

The spin-momentum locking naturally gives rise to various interesting spin-related physics. For example, charge fluctuations are naturally accompanied by spin fluctuations, leading to novel spin-plasmon excitations. ${ }^{215}$ ) The characteristic energy scale of such excitations is predicted to be a few meV, and those excitations may have been observed in ultra-highresolution ARPES experiments. ${ }^{216)}$ Also, shining a circularlypolarized light to the surface state selectively excites electrons with a particular spin polarization, ${ }^{217)}$ which means that electrons with a particular momentum direction is photo-excited in the TI surface state, and as a result, a photocurrent flows in a direction dictated by the light polarization. Such a peculiar photocurrent has actually been experimentally observed. ${ }^{218)}$

However, detection of a spin-polarized current on the surface of a TI turns out to be extremely difficult. This is because the spin-momentum locking makes the charge and spin scattering times to be the same, and therefore the spin diffusion length is equal to the electron mean free path in the TI surface state. ${ }^{219)}$ In such a situation, the spin polarization is significantly diminished in a diffusive transport (to the order of $\Delta k / k_{F}$, where $\Delta k$ is the shift of the Fermi surface induced by the applied electric field), ${ }^{220)}$ and the experiment must be done in a ballistic transport regime to detect a spin-polarized current. ${ }^{221)}$ No such experiment has been reported for 3D TIs, but in the $\mathrm{CdTe} / \mathrm{HgTe} / \mathrm{CdTe}$ quantum well in the $2 \mathrm{D} \mathrm{TI}$ regime, spin polarization of the edge current has been confirmed, ${ }^{70)}$ thanks to the long electron mean free path achievable in $\mathrm{HgTe}$ quantum wells.

\subsection{Dirac fermion physics}

When two spin-non-degenerate eigenstates forming a Kramers pair cross each other at a TRIM and the energy dispersion near this crossing point can be approximated by a linear dispersion, the low energy physics is described by massless Dirac equation. This means that a Kramers pair of surface states are actually forming a single 2D Dirac cone on which the spin degeneracy is lifted [Fig. 1(d)].

The Dirac equation for a free particle with mass $m$ is written as

$$
\begin{gathered}
E \psi(\mathbf{r})=c\left(\begin{array}{cc}
0 & \boldsymbol{\sigma} \\
\sigma & 0
\end{array}\right) \cdot \hat{\mathbf{p}} \psi(\mathbf{r})+m c^{2}\left(\begin{array}{cc}
I & 0 \\
0 & -I
\end{array}\right) \psi(\mathbf{r}) \\
=c\left(\begin{array}{cccc}
m c & 0 & \hat{p}_{z} & \hat{p}_{x}-i \hat{p}_{y} \\
0 & m c & \hat{p}_{x}+i \hat{p}_{y} & -\hat{p}_{z} \\
\hat{p}_{z} & \hat{p}_{x}-i \hat{p}_{y} & -m c & 0 \\
\hat{p}_{x}+i \hat{p}_{y} & -\hat{p}_{z} & 0 & -m c
\end{array}\right) \psi(\mathbf{r}),(111)
\end{gathered}
$$

where $\sigma$ is the vector of Pauli matrices and $\hat{\mathbf{p}}$ is the momentum operator. The energy eigenvalue of this equation is

$$
E= \pm c \sqrt{\mathbf{p}^{2}+m^{2} c^{2}},
$$


and hence the Dirac equation always has positive and negative energy states. One can easily see that this energy eigenvalue has a gap for a finite mass $m$, but it becomes gapless when $m=0$. This is the reason why a gapless system obeying the Dirac equation is called "massless"; it does not mean that the effective mass of electrons becomes zero. In fact, when the dispersion is linear, its second derivative is zero and the effective mass $m^{*}=\hbar^{2}\left(\partial^{2} E / \partial k^{2}\right)^{-1}$ diverges.

Graphene has emerged as a prototypical material to host 2D Dirac fermions. ${ }^{28)}$ Although both graphene and TIs are Dirac systems, there is an important difference. Namely, the former has both spin and valley ( $K$ and $K^{\prime}$ points in the BZ) degeneracies, while the latter is non-degenerate, and hence the Dirac fermion physics is simpler in TIs. This difference in degeneracy also means that the fermion degrees of freedom is $1 / 4$ in TIs compared to that in graphene.

A prominent property of Dirac fermions is that they carry the Berry phase of $\pi$, as was initially noted by Ando et al. ${ }^{55)}$ To see this, let us consider 2D massless Dirac fermions with the Fermi velocity $v_{F}$, for which the $4 \times 4$ matrix equation reduces to $2 \times 2$ and the Dirac equation is written as

$$
E \psi(\mathbf{r})=\hbar v_{F} \boldsymbol{\sigma} \cdot \hat{\mathbf{k}} \psi(\mathbf{r})=-i \hbar v_{F} \boldsymbol{\sigma} \cdot \nabla \psi(\mathbf{r}) .
$$

The eigenfunctions of this equation are

$$
\psi_{ \pm}(\mathbf{r})=\frac{1}{\sqrt{2}}\left(\begin{array}{c}
e^{-i \theta(\mathbf{k}) / 2} \\
\pm e^{i \theta(\mathbf{k}) / 2}
\end{array}\right) e^{i \mathbf{k} \cdot \mathbf{r}} \equiv u_{ \pm}(\mathbf{k}) e^{i \mathbf{k} \cdot \mathbf{r}}
$$

where $\theta(\mathbf{k})=\arctan \left(k_{y} / k_{x}\right)$, and the energy eigenvalues are

$$
E_{ \pm}= \pm \hbar v_{F} k
$$

When the wave vector $\mathbf{k}$ is adiabatically rotated anticlockwise along a closed path $C$ to encircle the origin, the Berry phase $\gamma$ acquired during this adiabatic cycle is

$$
\gamma=\oint_{C} d \mathbf{k} \cdot i\left\langle u_{ \pm}(\mathbf{k})\left|\nabla_{k}\right| u_{ \pm}(\mathbf{k})\right\rangle=\pi
$$

Because of this $\pi$ Berry phase, the time-reversed scattering paths, which in ordinary metals interfere constructively to cause the weak localization effect, ${ }^{222)}$ now destructively interfere each other, leading to the weak anti-localization effect. ${ }^{55)}$

Another prominent property of Dirac fermions is their peculiar Landau quantization of the energy states in magnetic fields. It has been shown ${ }^{52)}$ that the quantization occurs in the manner

$$
E_{ \pm}(N)= \pm \sqrt{\left(2 e \hbar v_{F}^{2} B / c\right) N}
$$

where $N=0,1,2, \ldots$ This means that in Dirac systems when the Fermi level is varied, the Landau-level (LL) spacing is not a constant but changes as $\sqrt{N}$, as opposed to ordinary metals in which the LL spacing is simply $\hbar \omega_{c}\left(=e \hbar B / m_{c} c\right.$ where $m_{c}$ is the cyclotron mass) and is independent of the Fermi level. Also, Eq. (117) indicates that there is the zeroth LL with $N=0$, which is pinned to the charge neutrality point (Dirac point). Therefore, the Landau quantization of massless

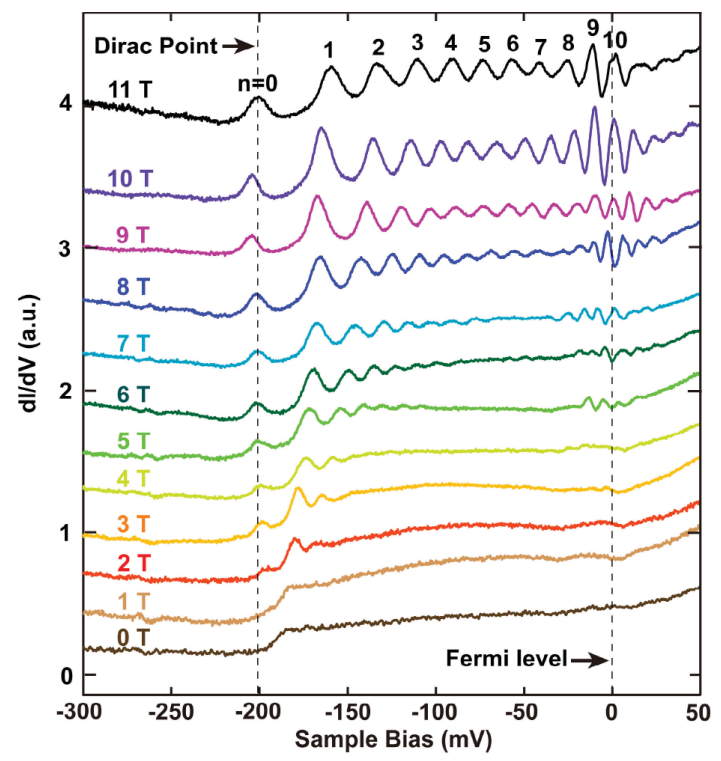

Fig. 14. (Color online) Landau quantization of the topological surface state observed in STS experiment on a 50-nm-thick $\mathrm{Bi}_{2} \mathrm{Se}_{3}$ film. The differential tunneling spectra were acquired for various magnetic fields from 0 to $11 \mathrm{~T}$ applied perpendicular to the surface. Taken from Ref. 174; copyright American Physical Society (2010).

Dirac fermions is characterized by the occurrence of the zeroenergy state and the symmetrical appearance of $\sqrt{N}$ states on both the positive and negative energy sides of the Dirac point. Such a peculiar Landau quantization of the surface state has indeed been observed in TIs by STS experiments in magnetic fields (Fig. 14). ${ }^{97,174,175)}$

When the Landau quantization of the form Eq. (117) takes place, the associated quantum Hall effect becomes unusual, and the Hall plateau between the $N$ th and $(N+1)$ th LLs is quantized to

$$
\sigma_{x y}=-\frac{e^{2}}{h}\left(N+\frac{1}{2}\right),
$$

which is called half-integer quantization [see Fig. 15]. ${ }^{28)}$ This unusual quantization can be understood to be a result of the existence of the $N=0 \mathrm{LL}$ at the Dirac point, which dictates that the first Hall plateaus on the positive and negative energy sides must appear antisymmetrically, because $\sigma_{x y}$ is an odd function of energy. The half-integer quantization can also be understood to be a result of the $\pi$ Berry phase, ${ }^{223,224)}$ which is most easy to see in the Laughlin's gauge argument ${ }^{8)}$ for the quantization of $\sigma_{x y}$.

\subsection{Quantum oscillations}

When the Landau quantization of the energy states occurs in crystalline solids in magnetic fields, the density of states (DOS) becomes periodically modulated as a function of magnetic field, which leads to various sorts of oscillation phenom- 

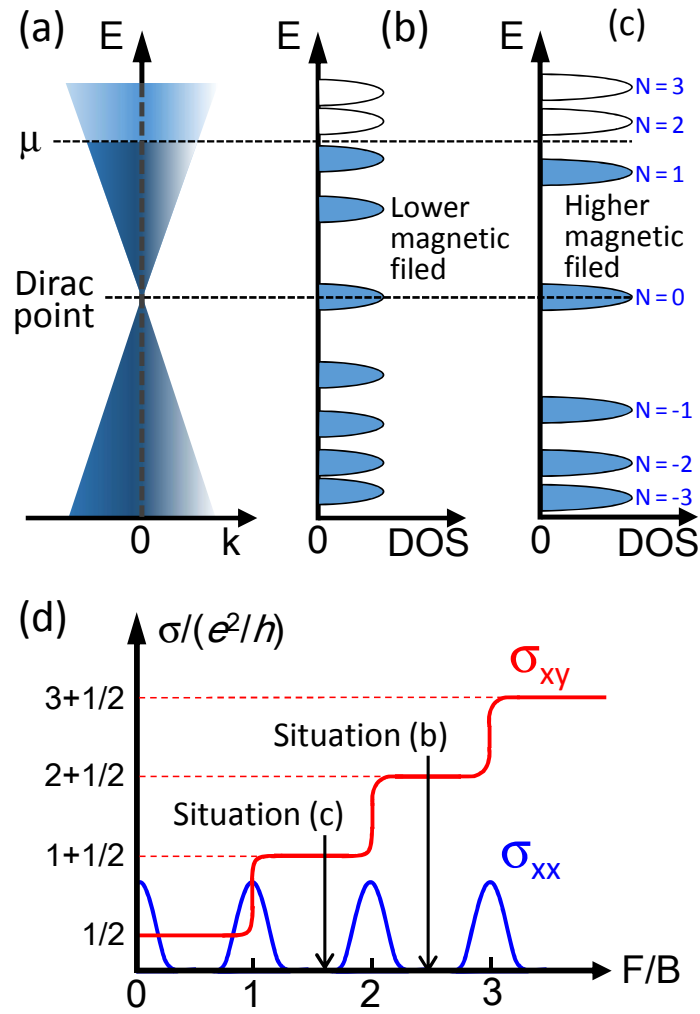

Fig. 15. (Color online) (a) Partially-filled 2D Dirac cone; $\mu$ is the Fermi level. (b) Landau quantization of the Dirac cone; LLs below $\mu$ are filled with electrons. Note that the spacing between LLs change as $\sqrt{N}$ and the $N=0$ LL is pinned to the Dirac point. (c) In a higher magnetic field, the spacing between LLs increases as $\sqrt{B}$, and fewer LLs are filled. (d) Schematic behavior of $\sigma_{x x}$ and $\sigma_{x y}$ in the quantum Hall regime of 2D Dirac fermions; the situations depicted in panels (b) and (c) are marked by arrows. Notice that the minima in $\sigma_{x x}$ marks a complete filling of up to $N$ th LLs.

ena that are generally called quantum oscillations. ${ }^{48)}$ In particular, the oscillations occurring in conductivity are called Shubnikov-de Haas $(\mathrm{SdH})$ oscillations, while the oscillations occurring in magnetic susceptibility are called de Haas-van Alphen (dHvA) oscillations.

$\mathrm{SdH}$ oscillations play particularly important roles in the studies of 3D TIs for two reasons. First, they provide a means to selectively and quantitatively characterize the $2 \mathrm{D}$ surface states that coexist with 3D bulk states. Second, the phase factor of the oscillations directly reflects the Berry phase of the system, which allows us to elucidate whether the electrons showing the $\mathrm{SdH}$ oscillations are Dirac fermions or not.

In $\mathrm{SdH}$ oscillations, conductivity oscillates periodically as a function of $1 / B$ and the oscillatory part of the longitudinal conductivity $\sigma_{x x}$ follows

$$
\Delta \sigma_{x x} \sim \cos \left[2 \pi\left(\frac{F}{B}-\frac{1}{2}+\beta\right)\right],
$$

where $F$ is the oscillation frequency and $\beta$ accounts for a phase shift $(0 \leq \beta<1)$. In fact, the same phase factor ap- pears in the Onsager's semiclassical quantization condition ${ }^{48)}$

$$
A_{N}=\frac{2 \pi e}{\hbar c} B\left(N+\frac{1}{2}-\beta\right)
$$

which is satisfied when the $N$ th LL is crossing the Fermi energy $E_{F}$. Here, $A_{N}$ is the area enclosed by electrons in the $k$-space with their cyclotron orbits on the Fermi surface. The parameter $\beta$ is simply the Berry phase $\gamma$ divided by $2 \pi$. For spinless fermions, it is known ${ }^{48,225)}$ that the Berry phase is zero for a parabolic energy dispersion $(\beta=0)$ and, as already noted, $\pi$ for Dirac fermions possessing a linear energy dispersion $\left(\beta=\frac{1}{2}\right)$. In real TI materials, the Dirac dispersion is not strictly linear but contain a parabolic component. Nevertheless, theoretically it has been elucidated that $\beta$ is robustly $\frac{1}{2}$ at least at large $N .^{226-228)}$

When $E_{F}$ lies at the center of a LL (which is usually broadened due to thermal fluctuations and disorder), the DOS takes a maximum; on the contrary, the DOS takes a minimum when $E_{F}$ lies in between two neighboring LLs [Fig. 15(b,c)]. In the latter situation, a certain number of LLs are completely filled and the next LL is empty. Therefore, a minimum in $\sigma_{x x}$, which occurs when DOS takes a minimum, signifies a complete filling of some $N$ LLs, and one can assign an integer index $N$ to that minimum. In ordinary metals, this LL index $N$ corresponds to the filling factor $v$. This can be easily understood by remembering the situation in the ordinary quantum Hall effect, in which $\sigma_{x y}$ is quantized to $v e^{2} / h$ and $\sigma_{x x}$ becomes zero when the chemical potential lies between the $v$ th and $(v+1)$ th LLs. (In the quantum Hall effect, the vanishing $\sigma_{x x}$ is a reflection of the bulk gap opening at the Fermi level.) On the other hand, in the case of Dirac fermions, the filling factor $v$ is not $N$ but is $N+\frac{1}{2}$ because of the half-integer quantization which essentially stems from the existence of the zeroth LL [Fig. 15(d)].

The phase factor $\beta$ in the $\mathrm{SdH}$ oscillations can be experimentally determined from an analysis of the so-called LL fan diagram, in which the sequence of the values of $1 / B_{N}$ corresponding to the $N$ th minimum in $\sigma_{x x}$ are plotted versus $N$. From Eq. (119), one can see that the $N$ th minimum occurs when the argument of the cosine equals $(2 N-1) \pi$, i.e.

$$
2 \pi\left(\frac{F}{B_{N}}-\frac{1}{2}+\beta\right)=(2 N-1) \pi .
$$

Therefore, the plot of $1 / B_{N}$ vs $N$ makes a straight line with a slope $F$ corresponding to the oscillation frequency. Note that $F / B_{1}=1-\beta$ holds for the 1 st minimum. Also, it follows from Eq. (121) that, when a linear fit to the LL fan diagram is extrapolated to $1 / B_{N} \rightarrow 0$, the intercept on the $N$-index axis gives the phase factor $\beta$. When the $\beta$ value thus obtained is $\frac{1}{2}$, one may conclude that the $\mathrm{SdH}$ oscillations come from Dirac fermions.

In the past, there have been confusions about the proper way to construct the LL fan diagram; namely, whether to assign an integer index to a minimum in $\rho_{x x}$ or to a minimum 
in $\sigma_{x x} \cdot{ }^{103,104,108,170,171,184,229-231)}$ Since it is important to clarify this confusion, let us discuss this issue in some detail. In solids, the resistivity tensor is an inverse of the conductivity tensor, and in the isotropic case their relation is

$$
\begin{aligned}
\left(\begin{array}{ll}
\rho_{x x} & \rho_{x y} \\
\rho_{y x} & \rho_{x x}
\end{array}\right) & =\left(\begin{array}{cc}
\sigma_{x x} & \sigma_{x y} \\
-\sigma_{x y} & \sigma_{x x}
\end{array}\right)^{-1} \\
& =\frac{1}{\sigma_{x x}^{2}+\sigma_{x y}^{2}}\left(\begin{array}{cc}
\sigma_{x x} & -\sigma_{x y} \\
\sigma_{x y} & \sigma_{x x}
\end{array}\right) .
\end{aligned}
$$

Therefore, when the condition $\sigma_{x x} \ll \sigma_{x y}$ is satisfied (which is usually the case with low-carrier-density semiconductors), $\rho_{x x} \simeq \sigma_{x x} / \sigma_{x y}^{2}$ and the minima in $\rho_{x x}$ coincide with those in $\sigma_{x x}$. This is the reason why the LL fan diagram constructed from the $\rho_{x x}$ data can give the correct phase factor in graphene. ${ }^{223,224)}$ However, in the case of TIs, due to the presence of the bulk transport channel, often the condition $\sigma_{x x} \ll \sigma_{x y}$ does not strictly hold. In the extreme case, when $\sigma_{x x} \gg \sigma_{x y}$ (which is usually the case with metals), $\rho_{x x} \simeq \sigma_{x x}^{-1}$ and the minima in $\rho_{x x}$ now coincide with the maxima in $\sigma_{x x}$. Therefore, unless $\sigma_{x x} \ll \sigma_{x y}$ is satisfied, performing the LL fan diagram analysis using the resistivity data is dangerous. Ideally, one should measure both $\rho_{x x}(B)$ and $\rho_{y x}(B)$ at the same time and convert them into $\sigma_{x x}(B)$ and $\sigma_{x y}(B)$ to perform reliable LL fan diagram analysis to elucidate the correct Berry phase.

In this regard, in the early stage of the TI research, the LL fan diagram analyses of the $\mathrm{SdH}$ oscillations observed in TIs were influenced by the case with graphene and used the minima in $\rho_{x x}$ for indexing integer $N .^{103,104,108,170,171,184,229-231 \text { ) }}$ Therefore, the conclusions regarding the Berry phase in those early publications should be taken with care. It was Xiong et al. who first switched to correctly using the minima in $\sigma_{x x}$ for the LL fan diagram analysis, ${ }^{105)}$ and some of the recent works performed reliable analyses and unambiguously demonstrated the Dirac nature of the surface state through determination of the Berry phase. ${ }^{105,109,172,173)}$ In addition, to determine the Berry phase from the LL fan diagram in a most reliable way, one should fix the slope of the linear fitting by using the frequency $F$ obtained from the Fourier analysis of the data; this way, the intercept on the $N$-index axis is obtained with little ambiguity. ${ }^{109,173)}$ An example of the LL fan diagram obtained for an MBE-grown $\mathrm{Bi}_{2} \mathrm{Se}_{3}$ film is shown in Fig. 16.

For the phase analysis of the $\mathrm{SdH}$ oscillations, an interesting lesson can be learned from the data for $\mathrm{Bi}_{2} \mathrm{Te}_{2} \mathrm{Se}$ published in 2010 by Ren et al. ${ }^{103)}$ In their original paper, they assigned integer index $N$ to the minima in $\rho_{x x}$ and obtained an intermediate phase factor $\beta$ of $0.22 \pm 0.12$ [see Fig. 17(b)]. ${ }^{103)}$ However, if the data are re-analyzed by calculating $\sigma_{x x}$ from $\sigma_{x x}=\rho_{x x} /\left(\rho_{x x}^{2}+\rho_{y x}^{2}\right)$ and identifying its minima to signify integer $N$, the same data set now gives $\beta=0.5$ [Fig. 17(c)], which reaffirms that Ren et al. were indeed observing $\mathrm{SdH}$ oscillations from Dirac fermions.

Besides the Berry phase, the $\mathrm{SdH}$ oscillations contain var- ious useful information. The Onsager's relation ${ }^{48)}$ gives $F$ in terms of the Fermi wave vector $k_{F}$ as

$$
F=(\hbar c / 2 \pi e) \pi k_{F}^{2}
$$

for a circular extremal cross section of FS, and therefore one can calculate the averaged $k_{F}$, and hence the carrier density, from the frequency of the $\mathrm{SdH}$ oscillations. To be explicit, for the topological 2D surface state the surface carrier density $n_{s}=\frac{1}{(2 \pi)^{2}} \pi k_{F}^{2}$ is directly obtained from $F$. If the surface state is of non-topological origin (such as accumulation or inversion layer), $n_{s}$ should be multiplied by 2 to account for the spin degeneracy. Also, if the $\mathrm{SdH}$ oscillations come from a 3D bulk state and the bulk Fermi surface is an ellipsoid, measurements of the $\mathrm{SdH}$ frequencies in magnetic fields along the three principal axes give the lengths of the three semiaxes, $k_{F}^{a}$,
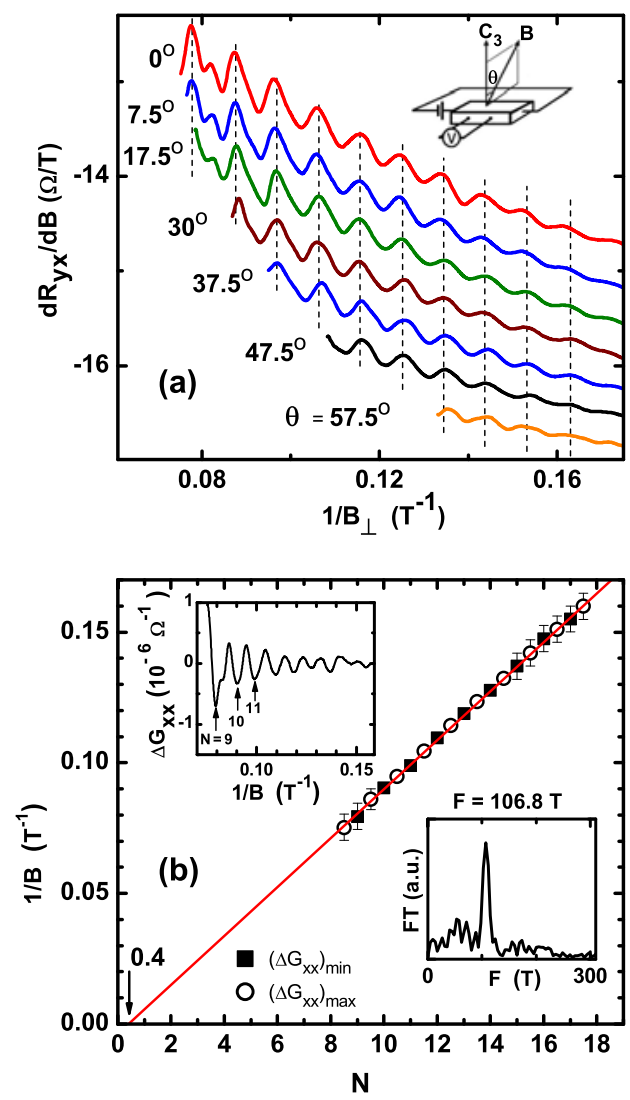

Fig. 16. (Color online) (a) $\mathrm{SdH}$ oscillations observed in a 10-nm-thick MBE-grown $\mathrm{Bi}_{2} \mathrm{Se}_{3}$ film; to enhance the visibility of the oscillations, $d R_{y x} / d B$ is plotted vs $1 / B_{\perp}(=1 / B \cos \theta)$ here. Notice that the positions of maxima and minima do not change with $1 / B_{\perp}$, which gives evidence for the $2 \mathrm{D}$ origin of the oscillations. (b) LL fan diagram constructed from the analysis of the $\sigma_{x x}$ data measured at $1.6 \mathrm{~K}$ and $\theta=0^{\circ}$; here, integer index $N$ are assigned to the minima (upper inset). Upon making a linear fitting to the data, the slope is fixed at the frequency $F$ obtained from the Fourier analysis of the data shown in the lower inset; the straight-line fitting extrapolates to $0.40 \pm$ 0.04 on the $N$-index axis, which is close to the value 1/2 expected for Dirac fermions. Based on the data from Ref. 173. 
$k_{F}^{b}$, and $k_{F}^{c}$; the bulk carrier density $n_{3 \mathrm{D}}$ for this Fermi surface is obtained as $n_{3 \mathrm{D}}=\left[2 /(2 \pi)^{3}\right](4 \pi / 3) k_{F}^{a} k_{F}^{b} k_{F}^{c}$, where the spin degeneracy is taken into account. ${ }^{204)}$

The 2D nature of the $\mathrm{SdH}$ oscillations can be tested by tak-
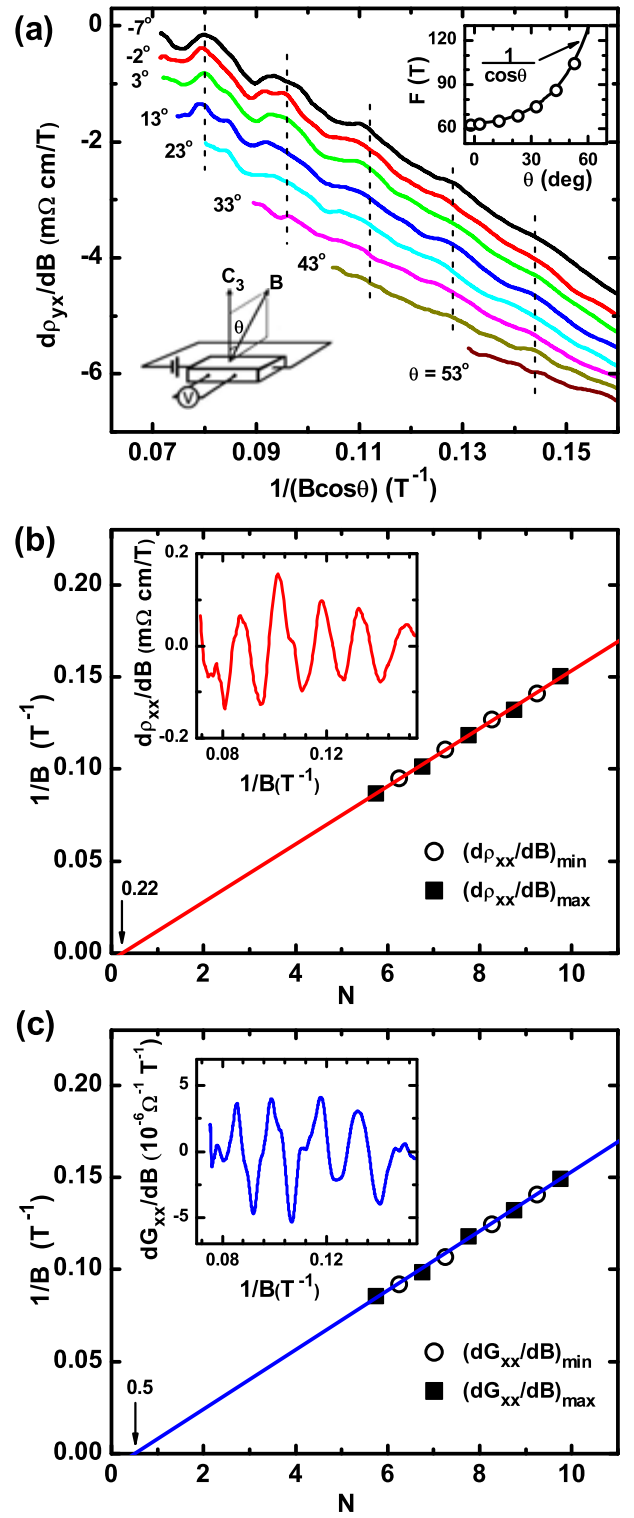

Fig. 17. (Color online) (a) $\mathrm{SdH}$ oscillations in $d \rho_{y x} / d B$ observed in a 260$\mu$ m-thick $\mathrm{Bi}_{2} \mathrm{Te}_{2} \mathrm{Se}$ single crystal; inset shows the magnetic-field angle dependence of the oscillation frequency $F$. (b) LL fan diagram based on the $d \rho_{x x} / d B$ data at $1.6 \mathrm{~K}$ and $\theta=0^{\circ}$ shown in the inset. Assuming that integer indices $N$ are to be assigned to the minima in $\rho_{x x}$, the minima and maxima in $d \rho_{x x} / d B$ correspond to $N+\frac{1}{4}$ and $N+\frac{3}{4}$, respectively. Linear fitting to the data extrapolates to $0.22 \pm 0.12$. (c) LL fan diagram based on the $d \sigma_{x x} / d B$ data shown in the inset; here, assuming that integer indices $N$ are to be assigned to the minima in $\sigma_{x x}, N+\frac{1}{4}$ and $N+\frac{3}{4}$ are assigned to the minima and maxima in $d \sigma_{x x} / d B$, respectively. Linear fitting to the data (in which the slope is constrained from the Fourier analysis) extrapolates to 0.5. Based on the data from Ref. 103. ing the dependence of $F$ on the angle $\theta$ between the magnetic field direction and the surface plane normal [Figs. 16(a) and 17(a)]; if the measured $F$ changes as $\sim 1 / \cos \theta$, it is a strong indication that the $\mathrm{SdH}$ oscillations come from a $2 \mathrm{D}$ system..$^{90,103,104,108,170,173)}$ One should note, however, that a reasonably wide range of $\theta$ (e.g., up to $50^{\circ}$ ) had better be measured, because $\mathrm{SdH}$ oscillations from an elongated 3D Fermi surface can present an approximate $1 / \cos \theta$ scaling for a limited range of $\theta .{ }^{104)}$ Also, disappearance of the SdH oscillations for $\theta=90^{\circ}$ gives additional support to the 2D nature.

The cyclotron mass $m_{c}$ of the carriers can be determined from the analysis of the temperature dependence of the amplitude of the SdH oscillations. This is because the quantum oscillations are expressed in the Lifshitz-Kosevich theory ${ }^{48)}$ as

$$
\Delta \sigma_{x x}=A_{0} R_{T} R_{D} R_{S} \cos \left[2 \pi\left(\frac{F}{B}-\frac{1}{2}+\beta\right)\right],
$$

where $A_{0}$ is a constant and the three coefficients, $R_{T}=2 \pi^{2}\left(k_{B} T / \hbar \omega_{c}\right) / \sinh \left[2 \pi^{2}\left(k_{B} T / \hbar \omega_{c}\right)\right], \quad R_{D}=$ $\exp \left[-2 \pi^{2}\left(k_{B} T_{D} / \hbar \omega_{c}\right)\right]$, and $R_{S}=\cos \left(\frac{1}{2} \pi g m_{e} / m_{c}\right)$ are called temperature, Dingle, and spin damping factors, respectively, ${ }^{48}$ ) with $T_{D}$ the Dingle temperature ( $g$ is the electron $g$-factor and $m_{e}$ is the free electron mass). In a fixed magnetic field, $R_{D}$ does not change and the temperature dependence shows up only through $R_{T}$; therefore, a fitting of the temperature dependence of the oscillation amplitude allows one to determine $\omega_{c}$, which in turn gives $m_{c}=e B /\left(c \omega_{c}\right)$. Remember, $m_{c}$ is defined as

$$
m_{c}=\frac{\hbar^{2}}{2 \pi}\left(\frac{\partial A(E)}{\partial E}\right)_{E=E_{F}},
$$

where $A(E)$ is the area enclosed by the cyclotron orbit in the $k$-space. Since the cyclotron orbit is confined on the Fermi surface in the $k$-space, the enclosed area changes with $E_{F}$. In the case of 2D Dirac fermions with the energy dispersion $E(k)=\hbar v_{F} k$, one obtains $A\left(E_{F}\right)=\pi k_{F}^{2}=\pi E_{F}^{2} /\left(\hbar v_{F}\right)^{2}$ and hence $m_{c}=E_{F} / v_{F}^{2}=\hbar k_{F} / v_{F}$. Thus, once $m_{c}$ is determined from the temperature dependence of the $\mathrm{SdH}$ amplitude, one can calculate $v_{F}=\hbar k_{F} / m_{c}$ and compare it with the slope of the Dirac dispersion known from the ARPES measurement to judge if the obtained $\mathrm{SdH}$ result is consistent with the known dispersion.

After $m_{c}$ is determined, one can further determine the Dingle temperature $T_{D}\left(=\hbar / 2 \pi k_{B} \tau\right)$ from the magnetic-field dependence of the amplitude of the $\mathrm{SdH}$ oscillations at fixed temperature and obtain the quantum scattering time $\tau$. This is usually done by making the Dingle analysis, in which one plots $\ln [A B \sinh (\alpha T / B)]$ against $1 / B(A$ is the observed magnetic-field-dependent amplitude of the oscillations and $\left.\alpha=14.7\left(m_{c} / m_{e}\right)[\mathrm{T} / \mathrm{K}]\right)$. Such a plot makes a straight line and its slope gives $T_{D}$ because of the relation $A=A_{0} R_{T} R_{D} R_{S} \sim$ $\left[\left(2 \pi^{2} k_{B} T / \hbar \omega_{c}\right) / \sinh \left(2 \pi^{2} k_{B} T / \hbar \omega_{c}\right)\right] \exp \left(-2 \pi^{2} k_{B} T_{D} / \hbar \omega_{c}\right)$.

The obtained $\tau$ is used for calculating the mean free path 
$\ell^{\mathrm{SdH}}=v_{F} \tau$, which in turn gives an estimate of the surface carrier mobility $\mu_{s}^{\mathrm{SdH}}=e \tau / m_{c}=e \ell^{\mathrm{SdH}} / \hbar k_{F}$.

Historically, the first observation of quantum oscillations coming from the $2 \mathrm{D}$ state of a 3D TI was made by Taskin and Ando in 2009 for $\mathrm{Bi}_{1-x} \mathrm{Sb}_{x}$, in which both $\mathrm{dHvA}$ and $\mathrm{SdH}$ oscillations were clearly observed. ${ }^{37)}$ In their experiment, up to five frequencies were resolved in the Fourier transform of the oscillations, and detailed angular dependence measurements of those frequencies in all three basal planes elucidated the existence of one circular 2D Fermi surface on the $C_{1}$ plane and three ellipsoidal electron pockets located at the $L$ points in the 3D BZ. Furthermore, it was possible to determine $m_{c}=0.0057 m_{e}$ and $T_{D}=6.7 \mathrm{~K}$ for the $2 \mathrm{D}$ channel from the analyses of the $2 \mathrm{D}$ component in the data. The $2 \mathrm{D}$ carrier density obtained from the oscillation frequency was only $n_{s}$ $=1.4 \times 10^{10} \mathrm{~cm}^{-2}$. The extremely small $m_{c}$ at very low $n_{s}$ is a characteristic feature of massless Dirac fermions, in which $m_{c}=E_{F} / v_{F}^{2}$; furthermore, the phase of the 2D component of the oscillations indicated the Berry phase of $\pi,{ }^{232}$ ) and hence the observed 2D carriers were most likely of topological origin. Nevertheless, it turned out that the $2 \mathrm{D}$ component of the oscillations is too strong if one assumes that the 2D carriers reside only on the outer surface of the sample, because the total number of bulk electrons was $\sim 10^{5}$ times larger than the total number of 2D carriers, and yet, the dHvA oscillations from the $2 \mathrm{D}$ carriers were as strong as those from bulk carriers. Although the actual situation in $\mathrm{Bi}_{1-x} \mathrm{Sb}_{x}$ is still not clear, what is probably happening is that $\mathrm{Bi}_{1-x} \mathrm{Sb}_{x}$ crystals contain a high density of planar defects along the crystallographic $C_{1}$ plane, and topological 2D states reside on such internal "surfaces".

The first observations of $\mathrm{SdH}$ oscillations coming really from the outer surface of $3 \mathrm{D}$ TIs were made for $\mathrm{Bi}_{2} \mathrm{Te}_{3}$ and $\mathrm{Bi}_{2} \mathrm{Se}_{3}$ by $\mathrm{Qu}$ et al. ${ }^{104)}$ and by Analytis et al. ${ }^{170)}$ respectively, at nearly the same time in 2010 . The $2 \mathrm{D}$ nature of the $\mathrm{SdH}$ oscillations were confirmed by taking the angular dependence of the oscillation frequency, as was the case with $\mathrm{Bi}_{1-x} \mathrm{Sb}_{x} .{ }^{37,90)}$ Since the surface band structures were already known from ARPES for those materials, ${ }^{94-96)}$ the agreement of $v_{F}$ estimated from the $\mathrm{SdH}$ data with that known from ARPES gave confidence in the origin of the observed 2D SdH oscillations. Those observations were made possible by minimizing the naturally-doped bulk carriers in $\mathrm{Bi}_{2} \mathrm{Te}_{3}$ and $\mathrm{Bi}_{2} \mathrm{Se}_{3}$, but still, the surface transport accounted for only less than $0.3 \%$ of the total conductance.

\subsection{Two-band analysis}

In real TI materials, the bulk transport channel is usually not negligible and one should consider parallel conductions through surface and bulk. Such a situation can be treated by a two-band model, in which the composite Hall resistivity is described as ${ }^{103)}$

$$
\rho_{y x}=\frac{\left(R_{s} \rho_{b}^{2}+R_{b} \rho_{s}^{2}\right) B+R_{s} R_{b}\left(R_{s}+R_{b}\right) B^{3}}{\left(\rho_{s}+\rho_{b}\right)^{2}+\left(R_{s}+R_{b}\right)^{2} B^{2}},
$$

where $R_{b}$ and $\rho_{b}$ are the Hall coefficient and resistivity of the bulk channel, $R_{s}=t /\left(e n_{s}\right)$, and $\rho_{s}=\rho_{\square} t$, with $t$ the sample thickness and $\rho_{\square}$ the surface sheet resistance. By fitting the $\rho_{y x}(B)$ data with this two-band model, one can obtain $n_{3 \mathrm{D}}(=$ $\left.1 / e R_{b}\right), \rho_{b}, n_{s}$, and $\rho_{\square}$, from which the mobilities for the bulk and surface channels are also obtained.

One should note that this analysis involves as many as four parameters. Nevertheless, the parameters must be consistent with the $\rho_{x x}$ value in zero field, which gives one constraint. If $\mathrm{SdH}$ oscillations are observed in the surface transport, the $\mathrm{SdH}$ frequency $F$ fixes the value of $n_{s}$, which is an additional constraint. With those two constraints, the two-band analysis becomes reasonably reliable.

For example, in the transport study of a $260-\mu \mathrm{m}$-thick $\mathrm{Bi}_{2} \mathrm{Te}_{2} \mathrm{Se}$ crystal reported by Ren et al. ${ }^{103)}$ observation of surface $\mathrm{SdH}$ oscillations (Fig. 17) allowed them to fix $n_{s}$, and the two-band analysis of $\rho_{y x}(B)$ (Fig. 18) gave consistent estimates of all the transport parameters: surface mobility $\mu_{s}^{\mathrm{tr}}=$ $1450 \mathrm{~cm}^{2} / \mathrm{Vs}$, bulk mobility $\mu_{b}=11 \mathrm{~cm}^{2} / \mathrm{Vs}, n_{s}=1.5 \times 10^{12}$ $\mathrm{cm}^{-2}$, and $n_{3 \mathrm{D}}=2.4 \times 10^{17} \mathrm{~cm}^{-3}$. These values are used for calculating the fraction of the surface contribution in the total conductance, which, in this example, was $\sim 6 \%$.

However, if the $\mathrm{SdH}$ oscillations are not observed, one should take the result of the two-band analysis with a grain of salt. This is because the model does not consider any magnetic-field dependence of the scattering rates, even though the magnetoresistance $\rho_{x x}(B)$ is usually large and complicated in TIs. In fact, it is almost always the case that the combination of parameters obtained from the two-band analyses of $\rho_{y x}(B)$ does not correctly reproduce the $\rho_{x x}(B)$ behavior.

It is often observed in TIs that the surface mobility $\mu_{s}^{\text {tr }}$ estimated from the two-band analysis is higher than $\mu_{s}^{\mathrm{SdH}}$ estimated from $\mathrm{SdH}$ oscillations. This discrepancy is most likely intrinsic and is a manifestation of the peculiar helical spin po-

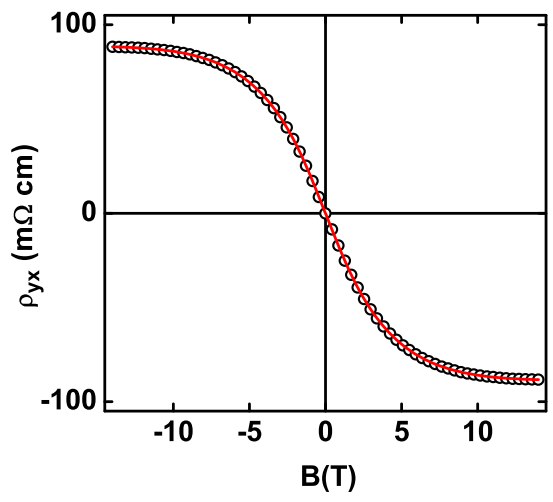

Fig. 18. (Color online) Two-band analysis of the non-linear $\rho_{y x}(B)$ data observed in $\mathrm{Bi}_{2} \mathrm{Te}_{2} \mathrm{Se}$ at $1.6 \mathrm{~K}$. Open circles are the data and the solid line is the fitting result. In this fitting, the surface carrier density $n_{s}$ is not a free parameter but is constrained from the $\mathrm{SdH}$ frequency. Based on the data from Ref. 103. 
larization. This is because $\tau$ obtained from $\mathrm{SdH}$ oscillations reflects scattering events in all directions equally, whereas in the transport coefficients such as $\rho_{y x}$ the backward scattering, which is discouraged in the TI surface states, plays the most important role. More specifically, $\tau^{\mathrm{tr}}$ to govern the resistivity acquires the additional factor $1 /(1-\cos \phi)$ upon spatial averaging ( $\phi$ is the scattering angle), while $\tau$ to govern the dephasing in the quantum oscillations is given by a simple spatial averaging without such a factor. ${ }^{233)}$ Hence, if the small-angle scattering becomes dominant (which is often the case at low temperature), $\tau^{\text {tr }}$ can be much larger than $\tau$.

\subsection{Weak anti-localization effect}

As briefly mentioned in conjunction with the Dirac fermion physics, the $\pi$ Berry phase associated with charge carriers leads to the weak anti-localization effect. ${ }^{55)}$ In the ordinary weak localization effect, ${ }^{222}$ electrons in metals are localized due to constructive interference of the electron wave functions between two time-reversed paths, which enhances the probability of those electrons to be found at a certain spatial location and reduces their ability to transport currents. This interference can be destroyed by breaking TRS with applied magnetic field, which shifts the phase factor of the wave functions of the two time-reversed paths differently. Therefore, when the weak localization is taking place, application of a magnetic field leads to a negative magnetoresistance, i.e., an enhancement of the conductivity. The anti-localization effect is just the opposite of this weak localization effect; ${ }^{55)}$ namely, because of the phase shift of $\pi$ which occurs when an electron travels along a closed path, the two time-reversed paths interfere destructively, reducing the probability of electrons to localize. Naturally, application of a magnetic field tends to lift this destructive interference, leading to a positive magnetoresistance.

It should be noted that this weak anti-localization effect also occurs in a system with strong SOC, ${ }^{234)}$ which causes a spin rotation whenever an electron is scattered off an impurity, and the resulting phase change of the electron wave function leads to the destructive interference similar to the case of the $\pi$ Berry phase. Therefore, the observation of the weak anti-localization effect alone does not give evidence for the existence of Dirac fermions.

The magnetic-field dependence of the conductivity for the weak anti-localization effect in 2D systems was calculated by Hikami, Larkin, and Nagaoka as ${ }^{234)}$

$$
\Delta \sigma_{x x}(B)=\alpha \frac{e^{2}}{\pi h}\left[\Psi\left(\frac{\hbar c}{4 e L_{\phi}^{2} B}+\frac{1}{2}\right)-\ln \left(\frac{\hbar c}{4 e L_{\phi}^{2} B}\right)\right],
$$

where $\Psi$ is the digamma function and $L_{\phi}$ is the phase coherence length. The prefactor $\alpha$ should be $-\frac{1}{2}$ for each transport channel that either carries the $\pi$ Berry phase or bears a strong SOC.

In $\mathrm{Bi}_{2} \mathrm{Se}_{3}$ thin films, this weak anti-localization effect is frequently observed and $\alpha \approx-\frac{1}{2}$ is usually found. This is
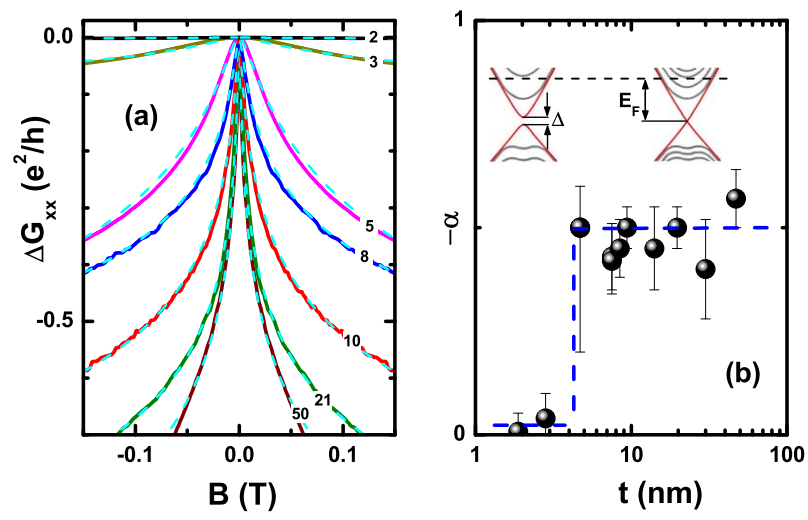

Fig. 19. (Color online) (a) Weak anti-localization behavior observed at 1.6 $\mathrm{K}$ in $\sigma_{x x}$ of a series of $\mathrm{MBE}$-grown $\mathrm{Bi}_{2} \mathrm{Se}_{3}$ thin films with various thickness shown in QL unit (1 QL is 0.95-nm thick); dashed lines are the fittings using the Hikami-Larkin-Nagaoka formula [Eq. (127)]. (b) Thickness dependence of the parameter $\alpha$ in Eq. (127). Inset shows schematic energy bands above and below the critical thickness at which hybridization of top and bottom surface states starts to develop. Taken from Ref. 173; copyright American Physical Society (2012).

because the top and bottom surfaces are connected though the bulk channel to form a single diffusive transport channel. ${ }^{173,186,235-237)}$ Remember, as long as the thickness is shorter than $L_{\phi}$ which is usually of the order of 100-1000 nm in TIs, electrons can diffusively travel from the bottom surface state to the top surface state though the bulk state using three different Fermi surfaces without losing the phase memory. Figure 19 shows an example of the weak anti-localization behavior observed in a series of MBE-grown $\mathrm{Bi}_{2} \mathrm{Se}_{3}$ thin films with varying thickness, in which $\alpha \approx-\frac{1}{2}$ was consistently observed unless the films are too thin.

Intriguingly, in films with low bulk-carrier density, by applying a gate voltage one can decouple one of the surface states from the rest through creation of an intervening gapped layer, and in such a situation $\alpha$ has been found to become -1 , signifying two independent diffusive transport channels. $^{235,236)}$

\subsection{Topological protection of the surface state}

An important property of the topological surface state of a TI is its topological protection. There are three aspects in the notion of topological protection. One is a consequence of the fundamental $Z_{2}$ topology which guarantees the existence of a gapless surface states as long as TRS is preserved. Another is a consequence of the helical spin polarization, which makes the spin eigenvalues of the states with $\mathbf{k}$ and $-\mathbf{k}$ to be exactly opposite; as a result, electrons with momentum $\mathbf{k}$ cannot be backscattered into the $-\mathbf{k}$ state because of the spin mismatch [Fig. 1(d)], and hence the surface state is protected from backscattering. (Note, however, that the scattering probability from $\mathbf{k}$ to $-\mathbf{k}+\delta$ is non-zero, and hence the surface state is not dissipationless.) The third is the $\pi$ Berry phase 
associated with massless Dirac fermions, ${ }^{55)}$ which protects them from weak localization through destructive interference of time-reversed paths. Those three effects collectively protect the topological surface state.

However, when TIs are thinned to the extent that the wave functions of the top and bottom surface states overlap, their hybridization leads to a gap opening at the Dirac point and results in a degenerate, massive Dirac dispersion. ${ }^{185,238-243)}$ For $\mathrm{Bi}_{2} \mathrm{Se}_{3}$, it was shown by ARPES experiments ${ }^{185,241)}$ that this hybridization gap opens in ultrathin films with the thickness of $6 \mathrm{~nm}$ or less.

Intriguingly, it was found by Taskin et al. in transport measurements of a series of high-quality $\mathrm{Bi}_{2} \mathrm{Se}_{3}$ thin films that the opening of the hybridization gap in the Dirac dispersion leads to a drastic diminishment of the metallic surface transport, which helps us understand the importance of topological protection in the transport properties of TIs. ${ }^{173)}$ Namely, in Fig. 19 one can see that the weak anti-localization behavior is quickly diminished in ultrathin films with thickness of less than $5 \mathrm{~nm}$, and this is because in such ultrathin films the opening of the hybridization gap causes the Berry phase $\gamma$ to be reduced from $\pi$. In the simplest case, $\gamma$ depends on the hybridization gap $\Delta$ as $^{244)}$

$$
\gamma=\pi\left(1-\frac{\Delta}{E_{F}}\right)
$$

This change in the Berry phase diminishes the weak antilocalization effect and the ordinary weak localization effect takes over. Indeed, it was demonstrated in Ref. 173 that $\rho_{x x}(T)$ of the 2- and 3-nm-thick films presented a localization behavior at low temperature, whereas all the films with thickness larger than $6 \mathrm{~nm}$ preserved a high surface mobility and presented pronounced SdH oscillations of Dirac nature. Therefore, the change in the transport properties in the hybridized regime signifies the consequences of the loss of topological protection. $^{173)}$

\subsection{Magnetic topological insulator}

Magnetic-ion doping to 3D TIs has been studied to see the effect of breaking TRS. Mn-doping to $\mathrm{Bi}_{2} \mathrm{Te}_{3}$ was reported to induce bulk ferromagnetism with Curie temperature of up to $12 \mathrm{~K}$ with $9 \% \mathrm{Mn}$ doping. ${ }^{245)}$ For $\mathrm{Bi}_{2} \mathrm{Se}_{3}$, it was reported that $\mathrm{Fe}$ and $\mathrm{Mn}$ dopings do not induce bulk ferromagnetism, but they were found to open a small gap at the Dirac point in the surface Dirac cone, ${ }^{246)}$ possibly because of a ferromagnetic order that develops only on the surface. Such a Dirac-fermion-mediated ferromagnetism was confirmed in Mn-doped $\mathrm{Bi}_{2}(\mathrm{Se}, \mathrm{Te})_{3}$ thin flakes, in which the chemical potential was successfully tuned into the bulk band gap so that the anomalous Hall effect coming only from the surface electrons can be measured to probe their ferromagnetic order. ${ }^{247)}$ Recently, in $\mathrm{Cr}$-doped ( $\mathrm{Bi}, \mathrm{Sb})_{2} \mathrm{Te}_{3}$ thin films, similar surface ferromagnetism was observed and, furthermore, when the chemical potential was tuned to be close to the Dirac point where a gap opens due to the ferromagnetic order, the quantum anomalous Hall effect with $\sigma_{x y}$ quantized to $e^{2} / h$ was observed at $30 \mathrm{mK}$, which signifies the appearance of a 1D chiral edge state as a consequence of the TRS breaking in the $2 \mathrm{D}$ topological surface state. ${ }^{248)}$

\section{Prospects}

There have been great improvements in the materials properties of TIs in the past few years to make them suitable for fundamental research. Now there are a couple of choices for bulk-insulating TI materials in which the surface transport dominates over residual bulk transport at low temperature in bulk single crystals. ${ }^{107,109)}$ High-quality thin film samples presenting pronounced $\mathrm{SdH}$ oscillations can also be grown, ${ }^{173,184)}$ which are useful for making devices to investigate various fundamental phenomena. In future, discoveries of new TI materials possessing bulk band gaps of more than $0.6 \mathrm{eV}$ would be desirable for room-temperature applications, but existing materials such as $\mathrm{Sn}$-doped $\mathrm{Bi}_{2} \mathrm{Te}_{2} \mathrm{Se}^{109)}$ are good enough for studying the topological surface transport at temperatures below $\sim 100 \mathrm{~K}$. It is expected that the focus of the TI research will gradually move to actually realizing novel topological phenomena using high-quality samples that have become available. In this regard, there are several major themes for future investigations, on which I elaborate below.

\subsection{Topological magnetoelectric effects}

The topological field theory for $Z_{2}$ TIs ${ }^{43)}$ leads to the appearance of an unusual $\mathbf{E} \cdot \mathbf{B}$ term in the Lagrangian

$$
\mathcal{L}=\frac{1}{8 \pi}\left(\epsilon \mathbf{E}^{2}-\frac{1}{\mu} \mathbf{B}^{2}\right)+\left(\frac{\alpha}{4 \pi^{2}}\right) \theta \mathbf{E} \cdot \mathbf{B},
$$

where $\epsilon$ is dielectric constant, $\mu$ is magnetic permeability, $\alpha=e^{2} / \hbar c$ is the fine structure constant, and $\theta=0$ or $\pi(\bmod$ $2 \pi$ ) is a topological invariant, which takes the value $\pi$ in $Z_{2}$ TIs. When $\theta$ is allowed to take any value, the second term (called $\theta$-term) describes the electrodynamics of an exotic fictitious particle axion. Due to the existence of the $\theta$-term, the constituent equations of a TI become

$$
\begin{aligned}
& \mathbf{D}=\mathbf{E}+4 \pi \mathbf{P}-\frac{\alpha \theta}{\pi} \mathbf{B} \\
& \mathbf{H}=\mathbf{B}-4 \pi \mathbf{M}+\frac{\alpha \theta}{\pi} \mathbf{E},
\end{aligned}
$$

where $\mathbf{D}$ is electric induction, $\mathbf{P}$ is electric polarization, and $\mathbf{M}$ is magnetization.

The most important consequence of this peculiar electromagnetism is that in TIs where $\theta=\pi$, electric field $\mathbf{E}$ induces magnetization $4 \pi \mathbf{M}=\alpha \mathbf{E}$ and the proportionality coefficient is a universal constant $\alpha$. Similarly, magnetic field $\mathbf{B}$ induces electric polarization $4 \pi \mathbf{P}=\alpha \mathbf{B}$. This is the topological magnetoelectric effect to characterize the nontrivial $Z_{2}$ topology of a TI in its electromagnetic properties. It should be noted, however, that the metallic surface states electrically short-circuit the whole surface, which makes it impossible to 
maintain electric field or polarization necessary for any magnetoelectric effects; therefore, to observe the topological magnetoelectric effect, one should gap out the surface states to avoid the short-circuiting effect. ${ }^{43,46)}$ This can, in principle, be done by depositing a ferromagnetic insulator whose magnetization is kept perpendicular to the surface. However, because of the practical difficulty to achieve such a gapping of the surface state, the topological magnetoelectric effect remains to be experimentally discovered. Even without opening a gap, magneto-optical properties of the surface state may show topological magnetoelectric effects in terms of a Faraday rotation quantized in integer multiples of $\alpha$ and a gigantic Kerr rotation of $\pi / 2 .{ }^{45}$ ) Another intriguing consequence of the $\theta$-term to be tested in future is the appearance of an image magnetic monopole; ${ }^{44)}$ namely, if the surface of a TI is gapped out by some means and a point charge is placed near the surface, the response of the TI looks as if there is a magnetic monopole in the TI.

\subsection{New types of topologies}

Widening the scope of topological materials is an important theme. Since superconductors have a superconducting gap at the Fermi level, they are in a way similar to insulators and one can conceive topological superconductors characterized by a topological invariant that is protected by the existence of a gap..$^{3,249)}$ So far, topological classifications of insulators and superconductors based on three discrete symmetries (TR, particle-hole, and chiral) have been established. ${ }^{4,250)} \mathrm{Re}$ cently, as mentioned in Sec. 4.5, new topological classifications based on point-group symmetry of the crystal lattice is attracting significant interest, ${ }^{166-169)}$ particularly after the new type of topological materials called topological crystalline insulators $^{61,161)}$ have been experimentally discovered. ${ }^{62,162,164)}$ Also, whereas it was thought to be necessary for topological materials to have a fully-gapped energy spectrum for a topological invariant to be well defined, ${ }^{4)}$ it is becoming possible to conceive a nontrivial topology for gapless systems. ${ }^{146,156,251-255)}$ It is an interesting time of topological expansion. Naturally, experimental discoveries of concrete materials that are nontrivial with respect to new topologies will continue to be crucially important.

\subsection{Majorana fermions}

Majorana fermions are exotic charge-neutral particles that are their own antiparticles. 5,256-258) While their existence in nature as elementary particles has not been confirmed since its prediction in 1937, recently its realizations in condensed matter as quasiparticles are attracting significant attention because of their fundamental novelty as well as their potential for being used as a qubit of fault-tolerant topological quantum computing. ${ }^{5)}$ To realize Majorana fermions in condensed matter, one must achieve particle-hole symmetry in a spin-non-degenerate system. ${ }^{256-258)}$ The former can be easily achieved in superconductors in which low-energy quasipar- ticles obey Bogoliubov-de Gennes equation characterized by inherent particle-hole symmetry; however, achieving a spinnon-degenerate superconductivity is a difficult task. ${ }^{259)}$

In this context, $\mathrm{Fu}$ and Kane realized that, since the topological surface state of TIs is spin non-degenerate, if superconductivity could be induced in the surface state by using proximity effect from an attached BCS superconductor, the resulting superconducting state harbors Majorana fermions. ${ }^{6}$ It is useful to note that such a proximity-induced superconducting state on the surface of a TI has singlet Cooper pairs, but nonetheless it is topologically nontrivial due to the $\pi$ Berry phase born by the surface Dirac electrons; therefore, such a surface can be considered a 2D topological superconductor. There have been a number of experimental reports to confirm the superconducting proximity effect in the topological surface states, ${ }^{171,231,260-268)}$ but the existence of Majorana fermions has not been elucidated.

Majorana fermions may also be found in a doped TI that becomes a superconductor. In this case, surface Dirac fermions may obtain superconductivity due to the proximity effect from the bulk and become a spin-non-degenerate topological superconductor. Another possibility is that the bulk superconductivity in a doped TI is itself topologically nontrivial (i.e. it is a bulk topological superconductor) due to strong SOC, ${ }^{269)}$ and its surface harbors dispersing, massless Majorana fermions. In this regard, the superconductor $\mathrm{Cu}_{x} \mathrm{Bi}_{2} \mathrm{Se}_{3},{ }^{270)}$ which is a doped 3D TI, has attracted a lot of attention. ${ }^{255,269,271-281)}$ In fact, $\mathrm{Cu}_{x} \mathrm{Bi}_{2} \mathrm{Se}_{3}$ was recently found to present signatures of unconventional superconductivity in its point-contact spectra, ${ }^{255)}$ and an unconventional superconductivity in this material is necessarily topological for symmetry reasons. ${ }^{255)}$

It should be mentioned that the experimental situation for $\mathrm{Cu}_{x} \mathrm{Bi}_{2} \mathrm{Se}_{3}$ is currently rather controversial. While some of the follow-up point-contact measurements ${ }^{276,277)}$ supported unconventional superconductivity originally reported by Sasaki et al. ${ }^{255)}$ a recent STS study ${ }^{278)}$ reported spectra that are consistent with conventional BCS superconductivity. This confusion essentially stems from the fact that available samples of superconducting $\mathrm{Cu}_{x} \mathrm{Bi}_{2} \mathrm{Se}_{3}$ are inhomogeneous ${ }^{279)}$ and the superconducting volume fraction never exceeds $70 \% ; 272,279$ ) furthermore, the author's group has found that one of the impurity phases is $\mathrm{CuSe}_{2}$, which is a conventional superconductor with the transition temperature of $2.4 \mathrm{~K}$. Obviously, improvements in the sample quality are desirable for local-probe measurements. Nevertheless, bulk superconducting properties like the temperature dependence of the upper critical field ${ }^{280}$ ) and the disorder dependence of the superfluid density ${ }^{281)}$ supported unconventional superconductivity in this material.

More recently, a superconducting doped TCI, $\mathrm{Sn}_{1-x} \mathrm{In}_{x} \mathrm{Te}$, was also found to present similar signatures of unconventional superconductivity in the point-contact spectroscopy, ${ }^{282)}$ and here again, an unconventional superconductivity is necessarily topological. ${ }^{282}$ ) Those bulk superconducting materials are intriguing candidates of topological superconductors to host 
massless Majorana fermions.

\subsection{Spintronics device applications}

The dissipationless spin current that exists in the topological surface state in equilibrium is expected to be useful for low energy consumption spintronic devices. Nevertheless, it is not clear how to exploit the dissipationless spin current for spintronic operations. This is obviously an important theme for both theory and experiment. When one breaks the equilibrium and push current through the surface state, the resulting current is spin polarized; however, as already discussed in Sec. 8.1, the expected spin polarization is extremely small in the diffusive regime and the experiment should be done in the ballistic transport regime, ${ }^{221}$ ) which has been difficult in practice. If successful, a useful feature of the current-induced spin polarization would be that the polarization direction can be easily switched by tuning the chemical potential across the Dirac point with gating.

\subsection{Hybrid structures based on TIs}

Once ideal samples of TIs become available, one can fabricate various types of hybrid structures involving TIs to exploit their novel properties. For example, unusual magneto-transport properties stemming from the peculiar spin-momentum locking on the surface of TIs have been theoretically predicted for TI-Ferromagnet hybrid structures. $^{283-285)}$ In this respect, recent report on the successful growth of the ferromagnetic insulator $\mathrm{EuS}$ on $\mathrm{Bi}_{2} \mathrm{Se}_{3}$ is an encouraging progress. ${ }^{286)}$ Also, combinations of a TI, a superconductor, and a ferromagnetic insulator would allow one to create and manipulate Majorana fermions. ${ }^{287-292)}$

\subsection{Electron interactions}

An important future direction is the merger of strong electron correlations and topology. There are already a number of theoretical works to elucidate the effect of electron correlations in TIs. ${ }^{293-298)}$ As the experiments on TIs become refined, the electron correlation physics will gradually be elucidated. Perhaps more importantly, if a material that becomes both insulating and topologically nontrivial because of strong electron correlations is discovered, such a materials would be called a topological Mott insulator ${ }^{299)}$ and will create an entirely new and rich field of research. There are theoretical discussions that oxides containing $5 d$ transition-metal elements might realize such a novel state of matter, ${ }^{300)}$ and discoveries along this line would be extremely interesting.

\section{Acknowledgments}

The author would like to thank T. Ando, L. Fu, H. Fukuyama, Y. Fuseya, A. Kapitulnik, D. Loss, N. Nagaosa, K. Nomura, S. Murakami, N. P. Ong, M. Sato, Y. Tanaka, and S.C. Zhang, for useful discussions and comments. Also, the author greatly acknowledges the contributions of his collaborators, K. Segawa, A. Taskin, Z. Ren, S. Sasaki, K. Eto, M. Kriener, T. Sato, S. Souma, T. Takahashi, I. Matsuda, F. Ko- mori, D. N. Basov, T. Kondo, S. Shin, T. Tsuda, and S. Kuwabata. The author acknowledges the support by JSPS (NEXT Program and KAKENHI 25220708), MEXT (Innovative Area "Topological Quantum Phenomena"), and AFOSR (AOARD 124038).

Yoichi Ando was born in Tokyo, Japan in 1964. He obtained B.Sc. (1987), M.Sc. (1989), and Ph.D. (1994) degrees from the University of Tokyo. He was a research scientist at the Central Research Institute of Electric Power Industry (CRIEPI, 1989-1991) and at the Superconductivity Research Laboratory, International Superconductivity Technology Center (SRL-ISTEC, 1991-1994), where he worked on his own toward his Ph.D. thesis. He did his postdoc at Bell Laboratories in the United States (1994-1996), and then returned to CRIEPI, where he led a research group as a senior research scientist (1996-2007) and also served as a department head (2004-2005). Since 2007, he has been a professor at the Institute of Scientific and Industrial Research, Osaka University. His research is aimed at discovery and understanding of novel quantum materials. To this end, he synthesizes new materials, grows high-quality single crystals, and performs top-notch measurements of various fundamental properties. He has made numerous contributions in the field of high- $T_{c}$ superconductivity by taking advantage of high-quality single crystals grown in his laboratory, but in addition, he has made a number of well-cited contributions to a thermoelectric material, a giant-magnetoresistance material, and a solid-oxide fuel cell material. Recently, he is most interested in revealing new physics in topological insulators and topological superconductors, and he has already contributed significantly to new materials discoveries of those materials. He has received the prestigious Japan Society for the Promotion of Science (JSPS) Prize in 2006, and also received the Superconductivity Science and Technology Award in 2003 and in 2013.

1) M. Z. Hasan and C. L. Kane: Rev. Mod. Phys. 82 (2010) 3045

2) J. E. Moore: Nature (London) 464 (2010) 194.

3) X.-L. Qi and S.-C. Zhang: Rev. Mod. Phys. 83 (2011) 1057.

4) A. P. Schnyder, S. Ryu, A. Furusaki, and A. W. W. Ludwig: Phys. Rev. B 78 (2008) 195125.

5) F. Wilczek: Nat. Phys. 5 (2009) 614.

6) L. Fu and C. L. Kane: Phys. Rev. Lett. 100 (2008) 096407.

7) K. v. Klitzing, G. Dorda, and M. Pepper: Phys. Rev. Lett. 45 (1980) 494.

8) R. B. Laughlin: Phys. Rev. B 23 (1981) 5632.

9) T. Ando and Y. Uemura: J. Phys. Soc. Jpn. 36 (1974) 959.

10) D. J. Thouless, M. Kohmoto, M. P. Nightingale, and M. den Nijs: Phys. Rev. Lett. 49 (1982) 405.

11) B. I. Halperin: Phys. Rev. B 25 (1982) 2185.

12) D. C. Tsui, H. L. Stormer, and A. C. Gossard: Phys. Rev. Lett. 48 (1982) 1559.

13) R. B. Laughlin: Phys. Rev. Lett. 50 (1983) 1395.

14) X. G. Wen and Q. Niu: Phys. Rev. B 41 (1990) 9377.

15) S. A. Wolf, D. D. Awschalom, R. A. Buhrman, J. M. Daughton, S. von Molnar, M. L. Roukes, A. Y. Chtchelkanova, and D. M. Treger: Science 294 (2001) 1488

16) M. I. D'yakonov and V. I. Perel': JETP Lett. 13 (1971) 467.

17) J. E. Hirsch: Phys. Rev. Lett. 83 (1999) 1834

18) S. Zhang: Phys. Rev. Lett. 85 (2000) 393.

19) S. Murakami, N. Nagaosa, and S. C. Zhang: Science 301 (2003) 1348.

20) J. Sinova, D. Culcer, Q. Niu, N. A. Sinitsyn, T. Jungwirth, and A. H. MacDonald: Phys. Rev. Lett. 92 (2004) 126603.

21) Y. K. Kato, R. C. Myers, A. C. Gossard, and D. D. Awschalom: Science 306 (2004) 1910.

22) N. Nagaosa, J. Sinova, S. Onoda, A. H. MacDonald, and N. P. Ong: Rev. Mod. Phys. 82 (2010) 1539.

23) S. Murakami, N. Nagaosa, and S.-C. Zhang: Phys. Rev. Lett. 93 (2004) 156804. 
24) M. Onoda and N. Nagaosa: Phys. Rev. Lett. 95 (2005) 106601.

25) C. L. Kane and E. J. Mele: Phys. Rev. Lett. 95 (2005) 226801.

26) C. L. Kane and E. J. Mele: Phys. Rev. Lett. 95 (2005) 146802.

27) B. A. Bernevig and S. C. Zhang: Phys. Rev. Lett. 96 (2006) 106802.

28) A. K. Geim and K. S. Novoselov: Nature Materials 6 (2007) 183.

29) A. H. Castro Neto, F. Guinea, N. M. R. Peres, K. S. Novoselov, and A. K. Geim: Rev. Mod. Phys. 81 (2009) 109.

30) B. A. Bernevig, T. L. Hughes, and S.-C. Zhang: Science 314 (2006) 1757.

31) M. König, S. Wiedmann, C. Brüne, A. Roth, H. Buhmann, L. W. Molenkamp, X.-L. Qi, and S.-C. Zhang: Science 318 (2007) 766.

32) J. E. Moore and L. Balents: Phys. Rev. B 75 (2007) 121306(R).

33) L. Fu, C. L. Kane, and E. J. Mele: Phys. Rev. Lett. 98 (2007) 106803

34) R. Roy: Phys. Rev. B 79 (2009) 195322

35) L. Fu and C. L. Kane: Phys. Rev. B 76 (2007) 045302.

36) D. Hsieh, D. Qian, L. Wray, Y. Xia, Y. S. Hor, R. J. Cava, and M. Z. Hasan: Nature 452 (2008) 970.

37) A. A. Taskin and Y. Ando: Phys. Rev. B 80 (2009) 085303.

38) P. Roushan, J. Seo, C. V. Parker, Y. S. Hor, D. Hsieh, D. Qian, A. Richardella, M. Z. Hasan, R. J. Cava, and A Yazdani: Nature 460 (2009) 1106.

39) D. Hsieh, Y. Xia, L. Wray, D. Qian, A. Pal, J. H. Dil, J. Osterwalder, F. Meier, G. Bihlmayer, C. L. Kane, Y. S. Hor, R. J. Cava, and M. Z. Hasan: Science 323 (2009) 919

40) A. Nishide, A. A. Taskin, Y. Takeichi, T. Okuda, A. Kakizaki, T. Hirahara, K. Nakatsuji, F. Komori, Y. Ando, and I. Matsuda: Phys. Rev. B. 81 (2010) 041309(R).

41) S. C. Zhang and J. P. Hu: Science 294 (2001) 823.

42) B. A. Bernevig, C. H. Chern, J. P. Hu, N. Toumbas, and S. C. Zhang: Ann. Phys. 300 (2002) 185.

43) X.-L. Qi, T. L. Hughes, and S.-C. Zhang: Phys. Rev. B 78 (2008) 195424.

44) X.-L. Qi, R. Li, J. Zang, and S.-C. Zhang: Science 323 (2009) 1184.

45) W.-K. Tse and A. H. MacDonald: Phys. Rev. B 82 (2010) 161104(R).

46) K. Nomura and N. Nagaosa: Phys. Rev. Lett. 106 (2011) 166802.

47) P. A. Wolff: J. Phys. Chem. Solids 25 (1964) 1057.

48) D. Shoenberg, Magnetic Oscillations in Metals (Cambridge University Press, Cambridge, 1984).

49) V. S. Edel'man: Sov. Phys. Usp. 20 (1977) 819

50) H. Fukuyama and R. Kubo: J. Phys. Soc. Jpn 28 (1970) 570.

51) M. H. Cohen and E. I. Blount: Phil. Mag. 5 (1960) 115.

52) J. W. McClure: Phys. Rev. 104 (1956) 666.

53) J. C. Slonczewski and P. R. Weiss: Phys. Rev. 109 (1958) 272.

54) G. W. Semenoff: Phys. Rev. Lett. 53 (1984) 2449.

55) T. Ando, T. Nakanishi, and R. Saito: J. Phys. Soc. Jpn. 67 (1998) 2857.

56) H. Fukuyama: J. Phys. Soc. Jpn. 76 (2007) 043711.

57) D. Kim, S. Cho, N. P. Butch, P. Syers, K. Kirshenbaum, S. Adam, J. Paglione and M. S. Fuhrer: Nature Physics 8 (2012) 459.

58) B. A. Volkov and O. A. Pankratov: Pis'ma Zh. Eksp. Teor. Fiz. 42 (1985) 145 [JETP Lett. 42 (1985) 178].

59) O. A. Pankratov, S. V. Pakhomov, and B. A. Volkov: Solid. State Commun. 61 (1987) 93.

60) E. Fradkin, E. Dagotto, and D. Boyanovsky: Phys. Rev. Lett. 57 (1986) 2967.

61) T. H. Hsieh, H. Lin, J. Liu, W. Duan, A. Bansil, and L. Fu: Nature Commun. 3 (2012) 982.

62) Y. Tanaka, Zhi Ren, T. Sato, K. Nakayama, S. Souma, T. Takahashi, K. Segawa, and Y. Ando: Nature Phys. 8 (2012) 800

63) O. Tchernyshyov: Phys. Rev. B 62 (2000) 16751.

64) M. V. Berry: Proc. R. Soc. London, Ser. A 392 (1984) 45.

65) This derivation follows the lecture note of K. Nomura (unpublished).

66) L. Fu and C. L. Kane: Phys. Rev. B 74 (2006) 195312.

67) R. Resta: J. Phys. Condens. Matter 22 (2010) 123201.

68) F. D. M. Haldane: Phys. Rev. Lett. 93 (2004) 206602.

69) A. Roth, C. Brüne, H. Buhmann, L. W. Molenkamp, J. Maciejko, X.-L.
Qi, and S.-C. Zhang: Science 325 (2009) 294.

70) C. Brüne, A. Roth, H. Buhmann, E. M. Hankiewicz, L. W. Molenkamp, J. Maciejko, X. L. Qi, and S. C. Zhang: Nature Phys. 8 (2012) 486.

71) M. König, M. Baenninger, A. G. F. Garcia, N. Harjee, B. L. Pruitt, C. Ames, P. Leubner, C. Brüne, H. Buhmann, L. W. Molenkamp, and D. Goldhaber-Gordon: Phys. Rev. X 3 (2013) 021003.

72) C. Liu, T. L. Hughes, X.-L. Qi, K. Wang, and S.-C. Zhang: Phys. Rev Lett. 100 (2008) 236601.

73) I. Knez, R. R. Du, and G. Sullivan: Phys. Rev. Lett. 107 (2011) 136603.

74) I. Knez, R.-R. Du, and G. Sullivan: Phys. Rev. Lett. 109 (2012) 186603.

75) L. Du, I. Knez, G. Sullivan, and R.-R. Du: arXiv:1306.1925.

76) I. Knez, R.-R. Du, and G. Sullivan: Phys. Rev. B 81 (2010) 201301(R)

77) S. Murakami: Phys. Rev. Lett. 97 (2006) 236805.

78) A. Shitade, H. Katsura, J. Kunes, X.-L. Qi, S.-C.Zhang, and N. Nagaosa: Phys. Rev. Lett. 102 (2009) 256403.

79) J. Balakrishnan, G. K. W. Koon, M. Jaiswal, A. H. Castro Neto, and B. Özyilmaz: Nature Phys. 9 (2013) 284.

80) J. Hu, J. Alicea, R. Wu, and M. Franz: Phys. Rev. Lett. 109 (2012) 266801.

81) T. Hirahara, G. Bihlmayer, Y. Sakamoto, M. Yamada, H. Miyazaki, S. Kimura, S. Blügel, and S. Hasegawa: Phys. Rev. Lett. 107 (2011) 166801.

82) F. Yang, L. Miao, Z. F. Wang, M.-Y. Yao, F. Zhu, Y. R. Song, M.-X Wang, J.-P. Xu, A. V. Fedorov, Z. Sun, G. B. Zhang, C. Liu, F. Liu, D Qian, C. L. Gao, and J.-F. Jia: Phys. Rev. Lett. 109 (2012) 016801.

83) C. Sabater, D. Gosalbez-Martinez, J. Fernandez-Rossier, J. G. Rodrigo, C. Untiedt, and J. J. Palacios: Phys. Rev. Lett. 110 (2013) 176802.

84) K. Takeda and K. Shiraishi: Phys. Rev. B 50 (1994) 14916.

85) M. Ezawa: Phys. Rev. Lett. 109 (2012) 055502.

86) B. Lenoir, M. Cassart, J.-P. Michenaud, H. Scherrer, and S. Scherrer: J. Phys. Chem. Solids 57 (1996) 89.

87) T. Hirahara, K. Miyamoto, I. Matsuda, T. Kadono, A. Kimura, T. Nagao, G. Bihlmayer, E. V. Chulkov, S. Qiao, K. Shimada, H. Namatame, M. Taniguchi, and S. Hasegawa: Phys. Rev. B 76 (2007) 153305

88) J. C. Y. Teo, L. Fu, and C. L. Kane: Phys. Rev. B. 78 (2008) 045426.

89) H.-J. Zhang, C.-X. Liu, X.-L. Qi, X.-Y. Deng, X. Dai, S.-C. Zhang, and Z. Fang: Phys. Rev. B 80 (2009) 085307.

90) A. A. Taskin, K. Segawa, and Y. Ando: Phys. Rev. B 82 (2010) 121302(R).

91) A. A. Schafgans, K. W. Post, A. A. Taskin, Y. Ando, X.-L. Qi, B. C Chapler, and D. N. Basov: Phys. Rev. B 85 (2012) 195440.

92) H. Zhang, C.-X. Liu, X.-L. Qi, X. Dai, Z. Fang, and S.-C. Zhang: Nat Phys. 5 (2009) 438.

93) C.-X. Liu, X.-L. Qi, H. Zhang, X. Dai, Z. Fang, and S.-C. Zhang: Phys. Rev. B 82 (2010) 045122.

94) Y. Xia, D. Qian, D. Hsieh, L. Wray, A. Pal, H. Lin, A. Bansil, D. Grauer, Y. S. Hor, R. J. Cava, and M. Z. Hasan: Nat. Phys. 5 (2009) 398.

95) Y. L. Chen, J. G. Analytis, J.-H. Chu, Z. K. Liu, S.-K. Mo, X. L. Qi, H. J. Zhang, D. H. Lu, X. Dai, Z. Fang, S. C. Zhang, I. R. Fisher, Z. Hussain, and Z.-X. Shen: Science 325 (2009) 178

96) D. Hsieh, Y. Xia, D. Qian, L. Wray, F. Meier, J. H. Dil, J. Osterwalder, L. Patthey, A. V. Fedorov, H. Lin, A. Bansil, D. Grauer, Y. S. Hor, R. J. Cava, and M. Z. Hasan: Phys. Rev. Lett. 103 (2009) 146401.

97) Y. Jiang, Y. Wang, M. Chen, Z. Li, C. Song, K. He, L. Wang, X. Chen X. Ma, and Q. K. Xue: Phys. Rev. Lett. 108 (2012) 016401.

98) L. Fu: Phys. Rev. Lett. 103 (2009) 266801.

99) T. Zhang, Peng Cheng, X. Chen, J.-F. Jia, X. Ma, K. He, L. Wang, H Zhang, X. Dai, Z. Fang, X. Xie, and Q.-K. Xue: Phys. Rev. Lett. 103 (2009) 266803.

100) Z. Alpichshev, J. G. Analytis, J.-H. Chu, I. R. Fisher, Y. L. Chen, Z. X Shen, A. Fang, and A. Kapitulnik: Phys. Rev. Lett. 104 (2010) 016401.

101) S. Souma, K. Kosaka, T. Sato, M. Komatsu, A. Takayama, T. Takahashi, M. Kriener, K. Segawa, and Y. Ando: Phys. Rev. Lett. 106 (2011) 216803.

102) S.-Y. Xu, L. A. Wray, Y. Xia, R. Shankar, A. Petersen, A. Fedorov, 
H. Lin, A. Bansil, Y. S. Hor, D. Grauer, R. J. Cava, and M. Z. Hasan: arXiv:1007.5111.

$103)$ Z. Ren, A. A. Taskin, S. Sasaki, K. Segawa, and Y. Ando: Phys. Rev. B 82 (2010) 241306(R).

104) D.X. Qu, Y. S. Hor, J. Xiong, R. J. Cava, and N. P. Ong: Science 329 (2010) 821.

105) J. Xiong, A. C. Petersen, D. Qu, Y. S. Hor, R. J. Cava, and N. P. Ong: Physica E 44 (2012) 917.

106) S. Jia, Huiwen Ji, E. Climent-Pascual, M. K. Fuccillo, M. E. Charles, J. Xiong, N. P. Ong, and R. J. Cava: Phys. Rev. B 84 (2011) 235206.

$107)$ Z. Ren, A.A. Taskin, S. Sasaki, K. Segawa, and Y. Ando, Phys. Rev. B 84 (2011) 165311

108) A. A. Taskin, Z. Ren, S. Sasaki, K. Segawa, and Y. Ando: Phys. Rev. Lett. 107 (2011) 016801

109) Z. Ren, A. A. Taskin, S. Sasaki, K. Segawa, and Y. Ando: Phys. Rev. B 85 (2012) 155301

110) T. Sato, K. Segawa, H. Guo, K. Sugawara, S. Souma, T. Takahashi, and Y. Ando: Phys. Rev. Lett. 105 (2010) 136802.

111) K. Kuroda, M. Ye, A. Kimura, S. V. Eremeev, E. E. Krasovskii, E. V. Chulkov, Y. Ueda, K. Miyamoto, T. Okuda, K. Shimada, H. Namatame, and M. Taniguchi: Phys. Rev. Lett. 105 (2010) 146801.

112) Y. L. Chen, Z. K. Liu, J. G. Analytis, J.-H. Chu, H. J. Zhang, B. H. Yan, S.-K. Mo, R. G. Moore, D. H. Lu, I. R. Fisher, S. C. Zhang, Z. Hussain, and Z.-X. Shen: Phys. Rev. Lett. 105 (2010) 266401.

113) B. Yan, C.-X. Liu, H.-J. Zhang, C.-Y. Yam, X.-L. Qi, T. Frauenheim, and S.-C. Zhang: EPL 90 (2010) 37002.

114) H. Lin, R. S. Markiewicz, L. A. Wray, L. Fu, M.Z. Hasan, and A. Bansil: Phys. Rev. Lett. 105 (2010) 036404

115) J. Vidal, X. Zhang, L. Yu, J.-W. Luo, and A. Zunger: Phys. Rev. B 84 (2011) 041109.

116) S.-Y. Xu, Y. Xia, L. A. Wray, S. Jia, F. Meier, J. H. Dil, J. Osterwalder, B. Slomski, A. Bansil, H. Lin, R. J. Cava, and M. Z. Hasan: Science 332 (2011) 560.

117) T. Sato, K. Segawa, K. Kosaka, S. Souma, K. Nakayama, K. Eto, T. Minami, Y. Ando, and T. Takahashi: Nat. Phys. 7 (2011) 840.

118) S. Souma, M. Komatsu, M. Nomura, T. Sato, A. Takayama, T. Takahashi, K. Eto, K. Segawa, and Y. Ando: Phys. Rev. Lett. 109 (2012) 186804.

119) M. Neupane, S.-Y. Xu, L. A. Wray, A. Petersen, R. Shankar, N. Alidoust, C. Liu, A. Fedorov, H. Ji, J. M. Allred, Y. S. Hor, T.-R. Chang, H.-T. Jeng, H. Lin, A. Bansil, R. J. Cava, and M. Z. Hasan: Phys. Rev. B 85 (2012) 235406.

120) K. Okamoto, K. Kuroda, H. Miyahara, K. Miyamoto, T. Okuda, Z. S. Aliev, M. B. Babanly, I. R. Amiraslanov, K. Shimada, H. Namatame, M. Taniguchi, D. A. Samorokov, T. V. Menshchikova, E. V. Chulkov, and A. Kimura: Phys. Rev. B 86 (2012) 195304.

121) S. Souma, K. Eto, M. Nomura, K. Nakayama, T. Sato, T. Takahashi, K. Segawa, and Y. Ando: Phys. Rev. Lett. 108 (2012) 116801.

122) H. Jin, J. H. Song, A. J. Freeman, and M. G. Kanatzidis: Phys. Rev. B 83 (2011) 041202(R)

123) T. V. Menshchikova, S. V. Eremeev, Yu. M. Koroteev, V. M. Kuznetsov, E. V. Chulkov: JETP Lett. 93 (2011) 15.

124) K. Kuroda, H. Miyahara, M. Ye, S. V. Eremeev, Yu. M. Koroteev, E. E. Krasovskii, E. V. Chulkov, S. Hiramoto, C. Moriyoshi, Y. Kuroiwa, K. Miyamoto, T. Okuda, M. Arita, K. Shimada, H. Namatame, M. Taniguchi, Y. Ueda, and A. Kimura: Phys. Rev. Lett. 108 (2012) 206803.

125) S. V. Eremeev, G. Landolt, T. V. Menshchikova, B. Slomski, Y. M. Koroteev, Z. S. Aliev, M. B. Babanly, J. Henk, A. Ernst, L. Patthey, A. Eich, A. A. Khajetoorians, J. Hagemeister, O. Pietzsch, J. Wiebe, R. Wiesendanger, P. M. Echenique, S. S. Tsirkin, I. R. Amiraslanov, J. H. Dil, and E. V. Chulkov: Nature Commun. 3 (2012) 635.

126) S. Muff, F. von Rohr, G. Landolt, B. Slomski, A. Schilling, R. J. Cava, J. Osterwalder, J. H. Dil: arXiv:1304.7648.

127) T. Valla, H. Ji, L. M. Schoop, A. P. Weber, Z.-H. Pan, J. T. Sadowski, E. Vescovo, A. V. Fedorov, A. N. Caruso, Q. D. Gibson, L. Müchler, C.
Felser, and R. J. Cava: Phys. Rev. B 86 (2012) 241101.

128) R. J. Cava, H. Ji, M. K. Fuccillo, Q. D. Gibson, and Y. S. Hor: J. Mater. Chem. C 1 (2013) 3176

129) C. Brüne, C. X. Liu, E. G. Novik, E. M. Hankiewicz, H. Buhmann, Y. L. Chen, X. L. Qi, Z. X. Shen, S. C. Zhang, and L. W. Molenkamp: Phys. Rev. Lett. 106 (2011) 126803.

130) K. Nakayama, K. Eto, Y. Tanaka, T. Sato, S. Souma, T. Takahashi, K. Segawa, and Y. Ando: Phys. Rev. Lett. 109 (2012) 236804.

131) B. Yan and S. C. Zhang: Rep. Prog. Phys. 75 (2012) 096501

132) A. Husmann, J. B. Betts, G. S. Boebinger, A. Migliori, T. F. Rosenbaum, and M. L. Saboungi: Nature 417 (2002) 421.

133) W. Zhang, R. Yu, W. Feng, Y. Yao, H. Weng, X. Dai, and Z. Fang: Phys Rev. Lett. 106 (2011) 156808

134) S. Lee, J. In, Y. Yoo, Y. Jo, Y. C. Park, H. J. Kim, H. C. Koo, J. Kim, B. Kim, and K. L. Wang: Nano Lett. 12 (2012) 4194.

135) A. Sulaev, P. Ren, B. Xia, Q. H. Lin, T. Yu, C. Qiu, S.-Y. Zhang, M.-Y Han, Z. P. Li, W. G. Zhu, Q. Wu, Y. P. Feng, L. Shen, S.-Q. Shen, L. Wang: AIP Advances 3 (2013) 032123.

136) T. Richter, C. Blömers, H. Lüth, R. Calarco, M. Indlekofer, M. Marso, and T. Schäpers: Nano Lett. 8 (2008) 2834.

137) M. Dzero, K. Sun, V. Galitski, and P. Coleman: Phys. Rev. Lett. 104 (2010) 106408

138) T. Takimoto: J. Phys. Soc. Jpn. 80 (2011) 123710.

139) Feng Lu, J. Zhao, H. Weng, Zhong Fang, and Xi Dai: Phys. Rev. Lett. 110 (2013) 096401.

140) X. Zhang, N. P. Butch, P. Syers, S. Ziemak, R. L. Greene, and J. Paglione: Phys. Rev. X 3 (2013) 011011.

141) S. Wolgast, C. Kurdak, Kai Sun, J. W. Allen, D.-J. Kim, Z. Fisk: arXiv: 1211.5104

142) J. Botimer, D.J. Kim, S. Thomas, T. Grant, Z. Fisk, Jing Xia: arXiv: 1211.6769

143) H. Miyazaki, T. Hajiri, T. Ito, S. Kunii, and S. Kimura: Phys. Rev. B 86 (2012) 075105

144) K. von Klitzing and G. Landwehr: Solid State Commun. 9 (1971) 2201

145) B. Rasche, A. Isaeva, M. Ruck, S. Borisenko, V. Zabolotnyy, B Büchner, K. Koepernik, C. Ortix, M. Richter, and J. van den Brink: Nature Mater. 12 (2013) 422

146) X. Wan, A. M. Turner, A. Vishwanath, and S. Y. Savrasov: Phys. Rev. B 83 (2011) 205101.

147) S. Chadov, X.-L. Qi, J. Kübler, G. H. Fecher, C. Felser, and S.-C. Zhang: Nat. Mater. 9 (2010) 541

148) H. Lin, L. A. Wray, Y. Xia, S. Xu, S. Jia, R. J. Cava, A. Bansil, and M. Z. Hasan: Nat. Mater. 9 (2010) 546

149) D. Xiao, Y. Yao, W. Feng, J. Wen, W. Zhu, X.-Q. Chen, G. M. Stocks, and Z. Zhang: Phys. Rev. Lett. 105 (2010) 096404.

150) W. Feng, D. Xiao, Y.Zhang, and Y. Yao: Phys. Rev. B 82 (2010) 235121

151) W. Al-Sawai, H. Lin, R. S. Markiewicz, L. A. Wray, Y. Xia, S.-Y. Xu, M. Z. Hasan, and A. Bansil: Phys. Rev. B 82 (2010) 125208.

152) C. Liu, Y. Lee, T. Kondo, E. D. Mun, M. Caudle, B. N. Harmon, S L. Bud'ko, P. C. Canfield, and A. Kaminski: Phys. Rev. B 83 (2011) 205133.

153) G. Goll, M. Marz, A. Hamann, T. Tomanic, K. Grube, T. Yoshino, and T. Takabatake: Physica B 403 (2008) 1065.

154) N. P. Butch, P. Syers, K. Kirshenbaum, A. P. Hope, and J. Paglione: Phys. Rev. B 84 (2011) 220504(R).

155) A. A. Burkov, M. D. Hook, and L. Balents: Phys. Rev. B 84 (2011) 235126.

156) A. M. Turner and A. Vishwanath: arXiv:1301.0330.

157) B.-J. Yang and Y. B. Kim: Phys. Rev. B 82 (2010) 085111

158) K. Ueda, J. Fujioka, Y. Takahashi, T. Suzuki, S. Ishiwata, Y. Taguchi, and Y. Tokura: Phys. Rev. Lett. 109 (2012) 136402.

159) G. B. Halasz and L. Balents: Phys. Rev. B 85 (2012) 035103.

160) B. Singh, A. Sharma, H. Lin, M. Z. Hasan, R. Prasad, and A. Bansil: Phys. Rev. B 86 (2012) 115208

161) L. Fu: Phys. Rev. Lett. 106 (2011) 106802. 
162) P. Dziawa, B. J. Kowalski, K. Dybko, R. Buczko, A. Szczerbakow, M. Szot, E. Lusakowska, T. Balasubramanian, B. M.Wojek, M. H. Berntsen, O. Tjernberg, and T. Story: Nat. Mater. 11 (2012) 1023.

163) S.-Y. Xu, C. Liu, N. Alidoust, D. Qian, M. Neupane, J. D. Denlinger, Y. J. Wang, L. A. Wray, R. J. Cava, H. Lin, A. Marcinkova, E. Morosan, A. Bansil, and M. Z. Hasan: arXiv:1206.2088.

164) S.-Y. Xu, C. Liu, N. Alidoust, M. Neupane, D. Qian, I. Belopolski, J.D. Denlinger, Y.J. Wang, H. Lin, L.A. Wray, G. Landolt, B. Slomski, J.H. Dil, A. Marcinkova, E. Morosan, Q. Gibson, R. Sankar, F.C. Chou, R.J. Cava, A. Bansil, and M.Z. Hasan: Nature Communications 3 (2012) 1192.

165) Y. Tanaka, T. Sato, K. Nakayama, S. Souma, T. Takahashi, Zhi Ren, M. Novak, K. Segawa, and Y. Ando: Phys. Rev. B 87 (2013) 155105

166) R.-J. Slager, A. Mesaros, V. Juricic, and J. Zaanen: Nat. Phys. 9 (2013) 98.

167) Y. Ueno, A. Yamakage, Y. Tanaka, and M. Sato: arXiv:1303.0202.

168) C.-K. Chiu, H. Yao, and S. Ryu: arXiv:1303.1843.

169) F. Zhang, C. L. Kane, and E. J. Mele: arXiv:1303.4144.

170) J. G. Analytis, R. D. McDonald, S. C. Riggs, J.-H. Chu, G. S. Boebinger, and I. R. Fisher: Nat. Phys. 6 (2010) 960.

171) B. Sacepe, J. B. Oostinga, J. L. Li, A. Ubaldini, N. J. G. Couto, E. Giannini, and A. F. Morpurgo: Nature Commun. 2 (2011) 575

172) J. Xiong, Y. Luo, Y. Khoo, S. Jia, R. J. Cava, and N. P. Ong: Phys. Rev. B 86 (2012) 045314.

173) A. A. Taskin, S. Sasaki, K. Segawa, and Y. Ando: Phys. Rev. Lett. 109 (2012) 066803.

174) P. Cheng, C. L. Song, T. Zhang, Y. Y. Zhang, Y. L. Wang, J. F. Jia, J. Wang, Y. Y. Wang, B. F. Zhu, X. Chen, X. Ma, K. He, L. L. Wang, X. Dai, Z. Fang, X. C. Xie, X. L. Qi, C. X. Liu, S. C. Zhang, and Q. K. Xue: Phys. Rev. Lett. 105 (2010) 076801.

175) T. Hanaguri, K. Igarashi, M. Kawamura, H. Takagi, and T. Sasagawa: Phys. Rev. B 82 (2010) 081305.

176) Q.-F. Sun, X. C. Xie, and J. Wang: Phys. Rev. B 77 (2008) 035327.

177) H. Steinberg, D. R. Gardner, Y. S. Lee, and P. Jarillo-Herrero: Nano Lett. 10 (2010) 5032.

178) J. G. Checkelsky, Y. S. Hor, R. J. Cava, and N. P. Ong: Phys. Rev. Lett. 106 (2011) 196801.

179) X. Chen, X.-C. Ma, K. He, J.-F. Jia, and Q.-K. Xue: Adv. Mater. 23 (2011) 1162.

180) T. Hirahara, Y. Sakamoto, Y. Saisyu, H. Miyazaki, S. Kimura, T. Okuda, I. Matsuda, S. Murakami, and S. Hasegawa: Phys. Rev. B 81 (2010) 165422 .

181) G. Zhang, H. Qin, J. Teng, J. Guo, Q. Guo, X. Dai, Z. Fang, and K. Wu: Appl. Phys. Lett. 95 (2009) 053114

182) H. D. Li, Z. Y. Wang, X. Kan, X. Guo, H. T. He, Z. Wang, J. N. Wang, T. L. Wong, N. Wang, and M. H. Xie: New J. Phys. 12 (2010) 103038.

183) N. Bansal, Y. S. Kim, E. Edrey, M. Brahlek, Y. Horibe, K. Iida, M. Tanimura, G.-H. Li, T. Feng, H.-D. Lee, T. Gustafsson, E. Andrei, and S. Oh: Thin Solid Films 520 (2011) 224.

184) M. Lang, L. He, F. Xiu, X. Yu, J. Tang, Y. Wang, X. Kou, W. Jiang, A V. Fedorov, and K. L. Wang: ACS Nano 6 (2012) 295

185) Y. Zhang, K. He, C.-Z. Chang, C.-L. Song, L.-L. Wang, X. Chen, J.-F. Jia, Z. Fang, X. Dai, W.-Y. Shan, S.-Q. Shen, Q. Niu, X.-L. Qi, S.-C. Zhang, X.-C. Ma, and Q.-K. Xue: Nat. Phys. 6 (2010) 584.

186) J. Chen, H. J. Qin, F. Yang, J. Liu, T. Guan, F. M. Qu, G. H. Zhang, J. R. Shi, X. C. Xie, C. L. Yang, K. H. Wu, Y. Q. Li, and L. Lu: Phys. Rev. Lett. 105 (2010) 176602

187) A. Richardella, D. M. Zhang, J. S. Lee, A. Koser, D. W. Rench, A. L. Yeats, B. B. Buckley, D. D. Awschalom, and N. Samarth: Appl. Phys. Lett. 97 (2010) 262104.

188) N. Bansal, Y. S. Kim, M. Brahlek, E. Edrey, and S. Oh: Phys. Rev. Lett. 109 (2012) 116804

189) A. A. Taskin, S. Sasaki, K. Segawa, and Y. Ando: Adv. Mater. 24 (2012) 5581.

190) X. F. Kou, L. He, F. X. Xiu, M. R. Lang, Z. M. Liao, Y. Wang, A. V.
Fedorov, X. X. Yu, J. S. Tang, G. Huang, X. W. Jiang, J. F. Zhu, J. Zou, and K. L. Wang: Appl. Phys. Lett. 98 (2011) 242102.

191) S. Schreyeck, N. V. Tarakina, G. Karczewski, C. Schumacher, T. Borzenko, C. Brüne, H. Buhmann, C. Gould, K. Brunner, and L. W. Molenkamp: Appl. Phys. Lett. 102 (2013) 041914

192) Y. Y. Li, G. Wang, X. G. Zhu, M. H. Liu, C. Ye, X. Chen, Y. Y. Wang, K. He, L. L. Wang, X. C. Ma, H. J. Zhang, X. Dai, Z. Fang, X. C. Xie, Y. Liu, X. L. Qi, J. F. Jia, S. C. Zhang, and Q. K. Xue: Adv. Mater. 22 (2010) 4002.

193) J. Zhang, C.-Z. Chang, Z. Zhang, J. Wen, X. Feng, K. Li, M. Liu, K He, L. Wang, X. Chen, Q.-K. Xue, X. Ma, and Y. Wang: Nat. Commun. 2 (2011) 574

194) H. Cao, R. Venkatasubramanian, C. Liu, J. Pierce, H. Yang, M. Z Hasan, Y. Wu, and Y. P. Chen: Appl. Phys. Lett. 101 (2012) 162104

195) A. Koma: J. Cryst. Growth 201/202 (1999) 236.

196) A. Ishida, T. Yamada, T. Tsuchiya, Y. Inoue, S. Takaoka, and T. Kita: Appl. Phys. Lett. 95 (2009) 122106.

197) A. A. Taskin, Satoshi Sasaki, Kouji Segawa, and Yoichi Ando: arXiv: 1305.2470

198) H. Peng, K. Lai, D. Kong, S. Meister, Y. Chen, X.-L. Qi, S.-C. Zhang, Z.-X. Shen, and Y. Cui: Nat. Mater. 9 (2010) 225.

199) D. Kong, W. Dang, J. J. Cha, H. Li, S. Meister, H. Peng, Z. Liu, and Y Cui: Nano Lett. 10 (2010) 2245

200) F. Xiu, L. He, Y. Wang, L. Cheng, L.-T. Chang, M. Lang, G. Huang, X. Kou, Y. Zhou, X. Jiang, Z. Chen, J. Zou, A. Shailos, and K. L. Wang: Nature Nanotech. 6 (2011) 216.

201) P. Gehring, B. F. Gao, M. Burghard, and K. Kern: Nano Lett. 12 (2012) 5137.

202) N. P. Butch, K. Kirshenbaum, P. Syers, A. B. Sushkov, G. S. Jenkins, H. D. Drew, and J. Paglione: Phys. Rev. B 81 (2010) 241301.

203) J. G. Analytis, J. H. Chu, Y. Chen, F. Corredor, R. D. McDonald, Z. X. Shen, and I. R. Fisher: Phys. Rev. B 81 (2010) 205407.

204) K. Eto, Z. Ren, A. A. Taskin, K. Segawa, and Y. Ando: Phys. Rev. B 81 (2010) 195309.

$205)$ D. O. Scanlon, P. D. C. King, R. P. Singh, A. de la Torre, S. M. Walker, G. Balakrishnan, F. Baumberger, and C. R. A. Catlow: Adv. Mater. 24 (2012) 2154.

206) L.-L. Wang, M. Huang, S. Thimmaiah, A. Alam, S. L. Bud'ko, A Kaminski, T. A. Lograsso, P. Canfield, and D. D. Johnson: Phys. Rev. B 87 (2013) 125303

207) J. G. Checkelsky, Y. S. Hor, M.-H. Liu, D.-X. Qu, R. J. Cava, and N. P Ong: Phys. Rev. Lett. 103 (2009) 246601

208) Z. Ren, A. A. Taskin, S. Sasaki, K. Segawa, and Y. Ando: Phys. Rev. B 84 (2011) 075316

209) O. B. Sokolov, S. Ya. Skipidarov, N. I. Duvankov, and G. G. Shabunina: J. Cryst. Growth 262 (2004) 442

210) H. Ji, J. M. Allred, M. K. Fuccillo, M. E. Charles, M. Neupane, L. A. Wray, M. Z. Hasan, and R. J. Cava: Phys. Rev. B 85 (2012) 201103.

211) P. Gehring, H. M. Benia, Y. Weng, R. Dinnebier, C. R. Ast, M. Burghard, and K. Kern: Nano Lett. 13 (2013) 1179.

212) T. Arakane, T. Sato, S. Souma, K. Kosaka, K. Nakayama, M. Komatsu, T. Takahashi, Z. Ren, K. Segawa, and Y. Ando: Nat. Commun. 3 (2012) 636.

213) D. Kong, Y. Chen, J. J. Cha, Q. Zhang, J. G. Analytis, K. Lai, Z. Liu, S. S. Hong, K. J. Koski, S.-K. Mo, Z. Hussain, I. R. Fisher, Z.-X. Shen, and Y. Cui: Nat. Nanotechnol. 6 (2011) 705.

214) S. S. Hong, J. J. Cha, D. Kong, and Y. Cui: Nature Commun. 3 (2012) 757.

215) S. Raghu, S. B. Chung, X.-L. Qi, and S.-C. Zhang: Phys. Rev. Lett. 104 (2010) 116401.

216) T. Kondo, Y. Nakashima, Y. Ota, Y. Ishida, W. Malaeb, K. Okazaki, S. Shin, M. Kriener, S. Sasaki, K. Segawa, Y. Ando: Phys. Rev. Lett. 110 (2013) 217601

217) D. Hsieh, F. Mahmood, J. W. McIver, D. R. Gardner, Y. S. Lee, and N Gedik: Phys. Rev. Lett. 107 (2011) 077401 
218) J. W. McIver, D. Hsieh, H. Steinberg, P. Jarillo-Herrero, and N. Gedik: Nature Nanotech. 7 (2012) 96.

219) C. Ojeda-Aristizabal, M. S. Fuhrer, N. P. Butch, J. Paglione, and I. Appelbaum: Appl. Phys. Lett. 101 (2012) 023102.

220) V. M. Edelstein: Solid State Commun. 73 (1990) 233.

221) A. A. Burkov and D. G. Hawthorn: Phys. Rev. Lett. 105 (2010) 066802.

222) P. A. Lee and T. V. Ramakrishnan: Rev. Mod. Phys. 57 (1985) 287.

223) K. S. Novoselov, A. K. Geim, S. V. Morozov, D. Jiang, M. I. Katsnelson, I. V. Grigorieva, S. V. Dubonos, and A. A. Firsov: Nature 438 (2005) 197.

224) Y. Zhang, Y.-W. Tan, H. L. Stormer, and P. Kim: Nature 438 (2005) 201.

225) G. P. Mikitik and Yu. V. Sharlai: Phys. Rev. Lett. 82 (1999) 2147.

226) A. A. Taskin and Y. Ando: Phys. Rev. B 84 (2011) 035301.

227) G. P. Mikitik and Yu. V. Sharlai: Phys. Rev. B 85 (2012) 033301.

228) A. R. Wright and R. H. McKenzie: Phys. Rev. B 87 (2013) 085411

229) H. Cao, J. Tian, I. Miotkowski, T. Shen, J. Hu, S. Qiao, and Y. P. Chen: Phys. Rev. Lett. 108 (2012) 216803.

230) L. He, F. Xiu, X. Yu, M. Teague, W. Jiang, Y. Fan, X. Kou, M. Lang, Y. Wang, G. Huang, N.-C. Yeh, and K. L. Wang: Nano Lett. 12 (2012) 1486.

231) M. Veldhorst, M. Snelder, M. Hoek, T. Gang, V. K. Guduru, X. L. Wang, U. Zeitler, W. G. van der Wiel, A. A. Golubov, H. Hilgenkamp, and A. Brinkman: Nat. Mater. 11 (2012) 417

232) In Fig. 2(c) of Ref. 37, the decomposed components of the oscillations are shown; the LL fan diagram constructed for the main component $f_{1}$, which comes from a 2D state, gives the phase factor $\beta$ of 0.5 .

233) S. Das Sarma and F. Stern: Phys. Rev. B 32 (1985) 8442

234) S. Hikami, A. I. Larkin, and Y. Nagaoka: Prog. Theor. Phys. 63 (1980) 707.

235) J. Chen, X. Y. He, K. H. Wu, Z. Q. Ji, L. Lu, J. R. Shi, J. H. Smet, and Y. Q. Li: Phys. Rev. B 83 (2011) 241304(R).

236) H. Steinberg, J. B. Laloë, V. Fatemi, J. S. Moodera, and P. JarilloHerrero: Phys. Rev. B 84 (2011) 233101.

237) Y. S. Kim, M. Brahlek, N. Bansal, E. Edrey, G. A. Kapilevich, K. Iida, M. Tanimura, Y. Horibe, S.-W. Cheong, and S. Oh: Phys. Rev. B 84 (2011) 073109.

238) J. Linder, T. Yokoyama, and A. Sudbo: Phys. Rev. B 80 (2009) 205401.

239) C.-X. Liu, H. Zhang, B. Yan, X.-L. Qi, T. Frauenheim, X. Dai, Z. Fang, and S.-C. Zhang: Phys. Rev. B 81 (2010) 041307(R).

240) H.-Z. Lu, W.-Y. Shan, W. Yao, Q. Niu, and S.-Q. Shen: Phys. Rev. B 81 (2010) 115407.

241) Y. Sakamoto, T. Hirahara, H. Miyazaki, S. Kimura, and S. Hasegawa: Phys. Rev. B 81 (2010) 165432.

242) K. Park, J. J. Heremans, V. W. Scarola, and D. Minic: Phys. Rev. Lett. 105 (2010) 186801.

243) J. Chang, L. F. Register, S. K. Banerjee, and B. Sahu: Phys. Rev. B 83 (2011) 235108.

244) H.-Z. Lu, J. Shi, and S.-Q. Shen: Phys. Rev. Lett. 107 (2011) 076801.

245) Y. S. Hor, P. Roushan, H. Beidenkopf, J. Seo, D. Qu, J. G. Checkelsky, L. A. Wray, D. Hsieh, Y. Xia, S.-Y. Xu, D. Qian, M. Z. Hasan, N. P. Ong, A. Yazdani, and R. J. Cava: Phys. Rev. B 81 (2010) 195203

246) Y. L. Chen, J.- H. Chu, J. G. Analytis, Z. K. Liu, K. Igarashi, H.- H. Kuo, X. L. Qi, S. K. Mo, R. G. Moore, D. H. Lu, M. Hashimoto, T. Sasagawa, S. C. Zhang, I. R. Fisher, Z. Hussain, and Z. X. Shen: Science 329 (2010) 659.

247) J. G. Checkelsky, J. Ye, Y. Onose, Y. Iwasa, and Y. Tokura: Nature Phys. 8 (2012) 729.

248) C.-Z. Chang, J. Zhang, X. Feng, J. Shen, Z. Zhang, M. Guo, K. Li, Y. Ou, P. Wei, L.-L. Wang, Z.-Q. Ji, Y. Feng, S. Ji, X. Chen, J. Jia, X. Dai, Z. Fang, S.-C. Zhang, K. He, Y. Wang, L. Lu, X.-C. Ma, and Q.-K. Xue: Science 340 (2013) 167

249) Y. Tanaka, M. Sato, and N. Nagaosa: J. Phys. Soc. Jpn. 81 (2012) 011013.

250) S. Ryu, A. P. Schnyder, A. Furusaki, and A. W. W. Ludwig: New J. Phys. 12 (2010) 065010
251) S. Ryu and Y. Hatsugai: Phys. Rev. Lett. 89 (2002) 077002.

252) M. Sato: Phys. Rev. B 73 (2006) 214502.

253) M. Sato and S. Fujimoto: Phys. Rev. Lett. 105 (2010) 217001.

254) M. Sato, Y. Tanaka, K. Yada, and T. Yokoyama: Phys. Rev. B 83 (2011) 224511.

255) S. Sasaki, M. Kriener, K. Segawa, K. Yada, Y. Tanaka, M. Sato, and Y. Ando: Phys. Rev. Lett. 107 (2011) 217001.

256) J. Alicea: Rep. Prog. Phys. 75 (2012) 076501.

257) C. W. J. Beenakker: Annu. Rev. Condens. Mat. Phys. 4 (2013) 113.

258) T. D. Stanescu and S. Tewari: J. Phys.: Condens. Matter 25 (2013) 233201.

259) N. Read and D. Green: Phys. Rev. B 61 (2000) 10267.

260) J. R. Williams, A. J. Bestwick, P. Gallagher, S. S. Hong, Y. Cui, A. S. Bleich, J. G. Analytis, I. R. Fisher, and D. Goldhaber-Gordon: Phys. Rev. Lett. 109 (2012) 056803.

261) L. Maier, J. B. Oostinga, D. Knott, C. Brüne, P. Virtanen, G. Tkachov, E M. Hankiewicz, C. Gould, H. Buhmann, and L. W. Molenkamp: Phys. Rev. Lett. 109 (2012) 186806.

262) P. Zareapour, A. Hayat, S. Y. F. Zhao, M. Kreshchuk, A. Jain, D. C Kwok, N. Lee, S.-W. Cheong, Z. Xu, A. Yang, G. D. Gu, S. Jia, R. J. Cava, and K. S. Burch: Nat. Commun. 3 (2012) 1056.

263) D. Zhang, J. Wang, A. M. DaSilva, J. S. Lee, H. R. Gutierrez, M. H. W. Chan, J. Jain, and N. Samarth: Phys. Rev. B 84 (2011) 165120.

264) F. Yang, Y. Ding, F. Qu, J. Shen, J. Chen, Z. Wei, Z. Ji, G. Liu, J. Fan, C. Yang, T. Xiang, and L. Lu: Phys. Rev. B 85 (2012) 104508.

265) F. Yang, F. Qu, J. Shen, Y. Ding, J. Chen, Z. Ji, G. Liu, J. Fan, C. Yang, L. Fu, and Li Lu: Phys. Rev. B 86 (2012) 134504

266) F. Qu, F. Yang, J. Shen, Y. Ding, J. Chen, Z. Ji, G. Liu, J. Fan, X. Jing, C. Yang, and Li Lu: Scientific Reports 2 (2012) 339

267) G. Koren and T. Kirzhner: Phys. Rev. B 86 (2012) 144508

268) S. Cho, B. Dellabetta, A. Yang, J. Schneeloch, Z. Xu, T. Valla, G. Gu, M. J. Gilbert, N. Mason: Nature Commun. 4 (2013) 1689.

269) L. Fu and E. Berg: Phys. Rev. Lett. 105 (2010) 097001

270) Y. S. Hor, A. J. Williams, J. G. Checkelsky, P. Roushan, J. Seo, Q. Xu, H. W. Zandbergen, A. Yazdani, N. P. Ong, and R. J. Cava: Phys. Rev. Lett. 104 (2010) 057001.

271) L. A. Wray, S.-Y. Xu, Y. Xia, Y. S. Hor, D. Qian, A. V. Fedorov, H. Lin, A. Bansil, R. J. Cava, and M. Z. Hasan: Nature Phys. 6 (2010) 855

272) M. Kriener, K. Segawa, Z. Ren, S. Sasaki, and Y. Ando: Phys. Rev. Lett. 106 (2011) 127004.

273) T. H. Hsieh and L. Fu: Phys. Rev. Lett. 108 (2012) 107005.

274) A. Yamakage, K. Yada, M. Sato, and Y. Tanaka: Phys. Rev. B 85 (2012) 180509(R).

275) L. Hao and T. K. Lee: Phys. Rev. B 83 (2011) 134516.

276) T. Kirzhner, E. Lahoud, K. B. Chaska, Z. Salman, and A. Kanigel: Phys Rev. B 86 (2012) 064517.

277) X. Chen, C. Huan, Y. S. Hor, C. A. R. Sa de Melo, and Z. Jiang: arXiv: 1210.6054

278) N. Levy, T. Zhang, J. Ha, F. Sharifi, A. A. Talin, Y. Kuk, and J. A. Stroscio: Phys. Rev. Lett. 110 (2013) 117001.

279) M. Kriener, K. Segawa, Z. Ren, S. Sasaki, S. Wada, S. Kuwabata, and Y. Ando: Phys. Rev. B 84 (2011) 054513.

280) T. V. Bay, T. Naka, Y. K. Huang, H. Luigjes, M. S. Golden, and A. de Visser: Phys. Rev. Lett. 108 (2012) 057001

281) M. Kriener, K. Segawa, S. Sasaki, and Y. Ando: Phys. Rev. B 86 (2012) 180505

282) S. Sasaki, Z. Ren, A. A. Taskin, K. Segawa, L. Fu, and Y. Ando: Phys Rev. Lett. 109 (2012) 217004

283) T. Yokoyama, J. Zang, and N. Nagaosa: Phys. Rev. B 81 (2010) 241410(R).

284) T. Yokoyama, Y. Tanaka, and N. Nagaosa: Phys. Rev. B 81 (2010) 121401(R).

285) T. Yokoyama and S. Murakami: Phys. Rev. B 83 (2011) 161407(R).

286) P. Wei, F. Katmis, B. A. Assaf, H. Steinberg, P. Jarillo-Herrero, D. Heiman, and J. S. Moodera: Phys. Rev. Lett. 110 (2013) 186807. 
287) L. Fu and C. L. Kane: Phys. Rev. Lett. 102 (2009) 216403.

288) A. R. Akhmerov, J. Nilsson, and C. W. J. Beenakker: Phys. Rev. Lett. 102 (2009) 216404.

289) Y. Tanaka, T. Yokoyama, and N. Nagaosa: Phys. Rev. Lett. 103 (2009) 107002.

290) K. T. Law, P. A. Lee, and T. K. Ng: Phys. Rev. Lett. 103 (2009) 237001

291) J. Linder, Y. Tanaka, T. Yokoyama, A. Sudbo, and N. Nagaosa: Phys. Rev. Lett. 104 (2010) 067001

292) T. Neupert, S. Onoda, and A. Furusaki: Phys. Rev. Lett. 105 (2010) 206404.

293) Y. Yamaji and M. Imada: Phys. Rev. B 83 (2011) 205122.

294) R. Peters, N. Kawakami, and T. Pruschke: Phys. Rev. Lett. 108 (2012)
086402.

295) T. Yoshida, S. Fujimoto, and N. Kawakami: Phys. Rev. B 85 (2012) 125113.

296) Y. Tada, R. Peters, M. Oshikawa, A. Koga, N. Kawakami, and S. Fujimoto: Phys. Rev. B 85 (2012) 165138.

297) T. Yoshida, R. Peters, S. Fujimoto, and N. Kawakami: Phys. Rev. B 87 (2013) 165109.

298) S. Ueda, N. Kawakami, and M. Sigrist: Phys. Rev. B 87 (2013) 161108.

299) S. Raghu, X.-L. Qi, C. Honerkamp, and S.-C. Zhang: Phys. Rev. Lett 100 (2008) 156401.

300) D. Pesin and L. Balents: Nat. Phys. 6 (2010) 376. 